


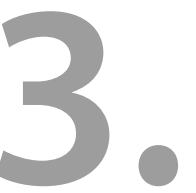

\section{Kongress der Deutschen Gesellschaft für Innere Medizin e.V.}

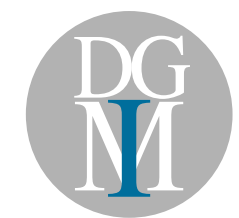

Internist $2017 \cdot 58$ (Suppl 1): S1-S48

DOI 10.1007/s00108-017-0235-y

Online publiziert: 06. April 2017

๑) Springer-Verlag Berlin Heidelberg 2017

\section{Wissenschaftliche Organisation}

\section{Kongress-Präsidentin}

Prof. Dr. med. Petra-Maria Schumm-Draeger

\section{DGIM-Kongress-Team 2017}

Dr. med. Alexandra Schoeneich

Dr. med. Julia Waldmann

Anne-Kathrin Wevers

Angela Hess

\section{Kongress-Sekretariat}

ZENTRUM / INNERE MEDIZIN / FÜNF HÖFE

Theatinerstraße 15

80333 München

Sandra Richardt

Telefon: + 49 (0) 89 - 443889222

Telefax: + 49 (0) 89 - 443889220

richardt@zim-fuenf-hoefe.de

\section{DGIM-Geschäftsstelle}

DG Irenenstraße 1

11. 65189 Wiesbaden

Geschäftsführung
RA Maximilian Broglie

Stellvertretende Geschäftsführerin

Dipl.-Kffr. Ourania Menelaou

Managementassistentin

Nicole Safenauer

Kongress-Sekretärin der DGIM

Katharina Wick

Verantwortlich für Patiententag

Dr. med. Norbert Schütz

Telefon: +49611 - 2058040 - 0

Telefax: +49611 - 2058040 - 46

info@dgim.de-www.dgim.de

Kongress Agentur (PCO)

m:con - mannheim:congress $\mathrm{GmbH}$

Thilo Hübner

Rosengartenplatz 2

D-68161 Mannheim

www.mcon-mannheim.de 


\section{3

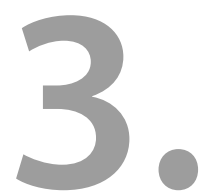 \\ Kongress der Deutschen Gesellschaft für Innere Medizin e.V.}

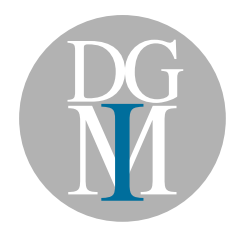

\section{Abstract-Gutachter DGIM 2017}

Diabetologie

Prof. Dr. med. Hendrik Lehnert, Lübeck

Prof. Dr. med. Petra-Maria Schumm-Draeger, München

Digitale Medizin

Prof. Dr. med. Ulrich R. Fölsch, Kiel

Prof. Dr. med. Gerd Hasenfuß, Göttingen

Endokrinologie

Prof. Dr. med. Felix Beuschlein, München

Prof. Dr. med. Hendrik Lehnert, Lübeck

Prof. Dr. med. Petra-Maria Schumm-Draeger, München Genderspezifische Aspekte der Inneren Medizin

Prof. Dr. med. Viola Hach-Wunderle, Frankfurt am Main Prof. Dr. med. Elisabeth Märker-Hermann, Wiesbaden Innere Medizin im höheren Lebensalter Prof. Dr. med. Michael D. Denkinger, UIm Prof. Dr. med. Hans-Jürgen Heppner, Schwelm Prof. Dr. med. Cornel C. Sieber, Nürnberg / Regensburg Palliativmedizin

Prof. Dr. med. Claudia Bausewein, München

Prof. Dr. med. Cornel C. Sieber, Nürnberg / Regensburg

Personalisierte Pharmakotherapie

Prof. Dr. med. Gabriel Eckermann, Kaufbeuren

Prof. Dr. med. Daniel Grandt, Saarbrücken

Präventivmedizin

Prof. Dr. med. Martin Halle, München

Prof. Dr. med. Hans Hauner, München

Schnittstelle Kardiologie / Adipositas / Diabetes

Prof. Dr. med. Johann Bauersachs, Hannover

Prof. Dr. med. Georg Ertl, Würzburg

Prof. Dr. med. Hans Hauner, München

Versorgungsforschung

Prof. Dr. med. Klaus G. Parhofer, München

Prof. Dr. med. Heiner Raspe, Münster

\section{Programmkomitee DGIM 2017}

Prof. Dr. med. Petra-Maria Schumm-Draeger, München (Vorsitzende)

Prof. Dr. med. Ayman Agah,München

Prof. Dr. med. Christian Albus, Köln

Prof. Dr. med. Johann Bauersachs, Hannover

Prof. Dr. med. Claudia Bausewein, München

Prof. Dr. med. Felix Beuschlein, München

Prof. Dr. med. Stephan C. Bischoff, Stuttgart

Prof. Dr. med. Reinhard Brunkhorst, Hannover

Prof. Dr. med. Michael Denkinger, Ulm

Prof. Dr. med. Georg Ertl, Würzburg

Priv.-Doz. Dr. med. Felix Gundling, München

Prof. Dr. med. Viola Hach-Wunderle, Frankfurt

Prof. Dr. med. Gerd Hasenfuß, Göttingen

Prof. Dr. med. Thomas Helmberger, München

Prof. Dr. med. Hans Heppner, Schwelm

Prof. Dr. med. Bernd Hertenstein, Bremen

Prof. Dr. med. Hans Hauner, München

Prof. Dr. med. Martin Halle, München

Angela Hess, München

Thilo Hübner, Mannheim

Priv.-Doz. Dr. med. Igor lesalnieks, München

PD Dr. med. Norma Jung, Köln

Dr. med. Matthias Kochanek, Köln

Prof. Dr. med. Stefan Kluge, Hamburg

Prof. Dr. med. Peter Kuhlencordt, München

Prof. Dr. med. Frank Lammert, Homburg

Prof. Dr. med. Hendrik Lehnert, Lübeck

Prof. Dr. med. Elisabeth Märker-Hermann, Wiesbaden

Prof. Dr. med. Ulf Müller-Ladner, Donaustauf

Prof. Dr. med. Michael Pfeifer, Donaustauf

Dr. Rupert Püllen, Frankfurt

Prof. Dr. med. Martin Reincke, München

Sandra Richardt, München

Prof. Dr. med. Andreas Saleh, München

Prof. Dr. med. Klemens Scheidhauer, München

Dr. med. Alexandra Schoeneich, München

Prof. Dr. med. Cornel Sieber, Regensburg/Nürnberg

PD Dr. med. Erhard Siegel, Heidelberg

Prof. Dr. med.Andreas Stallmach, Jena

Prof. Dr. med. Christian Strassburg, Hannover

Prof. Dr. med. Lorenz Trümper, Göttingen

Prof. Dr. med. J.-Christian Virchow, Rostock

Dr.med. Julia Waldmannm, München

Prof. Dr. med. Manfred Weber, Köln

Anne-Kathrin Wevers, München 
Internist 2017 $\cdot 58$ (Suppl 1): S1-S48

DOI 10.1007/s00108-017-0235-y

Online publiziert: 06. April 2017

๑) Springer-Verlag Berlin Heidelberg 2017

\section{Young Investigator Award}

\section{YIA 1}

\section{A comprehensive Analysis of RBM20 and Titin variants in Left} Ventricular Non-compaction Cardiomyopathy

Dr. Farbod Sedaghat-Hamedani'; Dr. Jan Haas; Feng Zhu;

Dr. Elham Kayvanpour; Christian Geier; Regina Pribe-Wolferts; Dr. Alan Lai; Karen Frese; Ali Amr; Martin Liss; Dr. Philipp Ehlermann; Christoph Dieterich; Yuhua Liao; Michael Gotthardt; Prof. Dr. Hugo A. Katus; Benjamin Meder ${ }^{1}$ Medizinische Universitätsklinik Heidelberg

Introduction: Left ventricular non-compaction cardiomyopathy (LVNC) is an increasingly recognized cause of heart failure, arrhythmia, thromboembolism, and sudden cardiac death, whose etiology is incompletely understood. We sought here to dissect the genetic causes and phenotypic presentation of LVNC in a large cohort.

Methods and Results: We assessed in total 95 LVNC patients (68 unrelated index patients and 27 affected relatives) by clinical phenotyping, cardiac imaging, molecular biomarkers and Illumina sequencing. $22.1 \%$ of the index patients presented with familial disease. Ventricular tachycardia (36.8\%) and thromboembolic events (10.3\%) were frequent findings. By conducting variant stratification and segregation analysis, we identified several novel candidate genes for LVNC. Besides a high percentage of Titin truncating variants (22.1\% TTNtv), we detected 14 rare variants in the RNA binding motif protein 20 (RBM20) gene. The novel, non-synonymous RBM2o variant p.R634L co-segregated in a LVNC family with autosomal dominant inheritance. The amino acid change affected the highly conserved arginine/serine-rich (RS) domain of RBM20. Since RBM2o has various splice targets, we performed mRNA sequencing of myocardial specimen of two affected patients, identifying a high percentage of exon inclusion in the I-band of Titin. Other known substrates of RBM20 were not significantly affected. By mRNA splicing assays, we showed a reduced splicing activity of the mutated construct, leading to reduced exon skipping in TTN mRNA. Finally, we successfully isolated protein from myocardial biopsies of the affected family members, revealing the predicted shift in Titin isoform expression towards a giant $\mathrm{N}_{2} \mathrm{BA}$ isoform.

Conclusion: In this study we report, for the first time, that reduced activity of RBM2o can lead to LVNC due to TTN missplicing. Furthermore, we were able to dissect the genetics of LVNC by whole-exome sequencing. Together, our findings can help in genetic counselling and management of affected patients and their families.

Key words: RBM20, non-compaction cardiomyopathy, Titin, Next-generation sequencing
YIA 2

Plasma-Osteopontin is associated with MRI-derived liver fat content but not with abdominal adipose tissue in a population-based sample

Marie-Christin Ritter'; Dr. Romina di Giuseppe ${ }^{2}$; Dr. Manja Koch²; Dr. Jan Borggrefe ${ }^{3}$; PD Dr. Hans-Peter Müller?; Prof. Dr. Jan Kassubek?; Dr. Gunnas Jacobs'; ; Dr. Gunnar Jacobs'; Dr. Matthias Lutz; Prof. Dr. Ute Nöthlings?; Prof. Dr. Wolfgang Lieb²; Prof. Dr. Wolfgang Lieb; Prof. Dr. Norbert Frey ${ }^{1 / 1}$

${ }^{1}$ University Hospital Schleswig-Holstein; ${ }^{2}$ Christian-Albrechts-University of Kiel; ${ }^{3}$ University-Clinics Cologne; ${ }^{4}$ University of Ulm; ${ }^{5}$ University of Bonn

Background: Osteopontin (OPN) emerged as a potential biomarker of cardiovascular diseases in experimental and clinical settings. However, little is known about clinical correlates of circulating OPN levels and their association with subclinical cardiometabolic traits in the general population. Therefore, we aimed to relate OPN to a broad spectrum of cardiovascular risk factors and cardiometabolic traits, including subcutaneous and visceral abdominal adipose tissue as well as liver fat content (LFC) in a community-based sample from Northern Germany.

Methods: In this cross-sectional study, OPN concentrations were measured in 416 individuals from the general population (popgen control cohort; 1 st follow-up visit; mean age $67 \pm 7$ years; $42 \%$ women). Participants received anthropometric measurements and comprehensive physical examination, assessment of standard cardiovascular risk factors and whole body MRI measurements. Fasting blood samples were stored at $-80^{\circ} \mathrm{C}$ until OPN was measured in plasma using ELISA. Visceral abdominal (VAT), subcutaneous abdominal (SAT) and subcutaneous trunk (STRAT) adipose tissue were quantified from a T1 weighted MRI sequence using ATLAS software and ARTIS algorithm. LFC was measured in a chemical shift imaging MRI sequence utilizing ImageJ software. Clinical correlates of OPN were identified by Spearman correlation coefficients and linear regression models with backward selection (candidate variables included age, sex, years of education (?11; <11 years), BMI, systolic blood pressure, antihypertensive drugs, $\mathrm{HbA1c}$, CRP, triglycerides, HDL, LFC, daily alcohol consumption, smoking (former; current smoker), pack years and physical activity). Linear and non-linear associations of OPN with SAT, STRAT, VAT and LFC were evaluated by means of restricted cubic spline regression models.

Results: Median OPN concentrations were $394.2 \mathrm{ng} / \mathrm{ml}$ (325.3-500.4 $\mathrm{ng} / \mathrm{ml}$ ) in men and $380.5 \mathrm{ng} / \mathrm{ml}(297.0-481.3 \mathrm{ng} / \mathrm{ml})$ in women. In multi- 
variable-adjusted models, log-transformed OPN levels were independently and inversely associated with triglycerides $(B=-0.06$ per 1-unit increase; $p=0.02), \mathrm{HDL}(\beta=-0.10, p=0.03)$ and former smoking $(\beta=-0.08$, $\mathrm{p}=0.03)$. The relation between LFC and OPN was J-shaped with both, very low as well as high biomarker levels being associated with higher liver fat values. The relationship between OPN and LFC was independent from potential confounders investigated in this study $(\beta=-0.02$, $p=0.02)$. OPN levels were not associated with SAT ( $\beta=-0.001, p=0.36)$, STRAT ( $(=-0.001, p=0.65)$ and VAT $(\beta=0.001, p=0.53)$.

Conclusion: We identified triglycerides, HDL and former smoking as important correlates of circulating OPN in multivariable-adjusted models. In a restricted cubic spline regression model plasma OPN displayed a J-shaped association with LFC as determined by MRI. Further studies are needed to confirm our findings and to assess the clinical utility of OPN screening for cardiometabolic risk stratification.

\section{YIA 3}

Inhibition of the JAK/STAT pathway: a novel approach in the treatment of acute myeloid leukemia

Jan Habbel; Dr. Lothar Vassen; Prof. Dr. med. Ulrich Dührsen; Dr. med. Maher Hanoun

Universitätsklinikum Essen, Klinik für Hämatologie

Background: Acute myeloid leukemia (AML) is a heterogeneous hematologic malignancy, characterized by high relapse rates and poor long-term outcome. With an average age of about 67 years it is a disease of the elderly. The bone marrow (BM) niche plays a pivotal role in AML pathogenesis. Dysregulation of the BM niche includes an aberrant production of inflammatory signals. We could show that levels of pro-inflammatory IL-6 are elevated in primary human BM aspirates while anti-inflammatory IL-10 is decreased, which strongly supports the concept of an inflammatory niche in AML. As inflammation is mediated through the JAK/STAT pathway we evaluated the efficacy of the selective JAK1 12 inhibitor INCB18424.

Methods: We established a serum-free co-culture model for primary human AML cells which revealed a significant stroma-mediated support of AML cell growth. In monoculture INCB18424 significantly inhibits AML cell proliferation (-48.9\%). Strikingly, treating co-cultured AML cells INCB18424 overcomes stromal support of AML progression, resulting in an even stronger anti-leukemic effect (-55.3\%). Separating myeloid blasts from non-leukemic cells by FACS from one patient sample substantiated the specific anti-leukemic effects of INCB18424 while sparing healthy hematopoietic cells. To reveal the underlying mechanisms we checked for phosphorylation of STAT3 in primary human AML cells. Neither the steady state phosphorylation nor the responsiveness to inflammatory signals differed significantly in AML and healthy blood cells. We therefore concluded that the effect cannot solely be STAT3 dependent. Next we performed microarray analyses which revealed that the anti-leukemic effects of INCB18424 are due to inhibition of cell cycle and induction of apoptosis which could be confirmed in a series of AML cell lines and primary cells. Further, the reactive oxygen species (ROS) pathway revealed to be a target of INCB18424. We could demonstrate that stroma cells strongly induce ROS in human leukemic cells, which has been previously shown to promote leukemogenesis. INCB18424 proved to target inflammatory signaling in stroma cells and efficiently reduced ROS levels in AML cells $(p=0.017)$. The microenvironment not only promotes AML progression, but further contributes to drug resistance in leukemia. INCB18424 revealed to partially rescue the stroma-mediated chemoresistance of cytarabine on primary leukemic cells $(p=0.029)$.

Results: In summary, our data identify that leukemic cells remodel the BM niche into a pro-inflammatory phenotype, creating a microenvironment that favors AML progression. Treatment with INCB18424 shows a strong cell-autonomous effect. Moreover, it disrupts the crosstalk of AML cells with their microenvironment, leading to striking anti-leukemic effects, overcoming stroma-mediated chemoresistance while sparing healthy hematopoiesis. INCB18424 is a promising novel approach in niche-targeted therapies for older patients who are not eligible for aggressive treatment regimens.

\section{YIA 4}

Bedeutung der Adiponektin-Subfraktionen als Prognosemarker bei chronisch nierenkranken Menschen

Angelika Kircher'; Prof. Dr. Gunnar H. Heine; PD Dr. Sarah Seiler;

Dr. med. Insa Emrich; Prof. Dr. med. Danilo Fliser

${ }^{1}$ Universität des Saarlandes

Hintergrund: Das Peptidhormon Adiponektin zirkuliert als „low molecular weight" (LMW) Trimer, als "middle molecular weight" (MMW) Hexamer sowie als "high molecular weight" (HMW) Multimer. Studien zeigten, dass LMW und MMW Adiponektin eine antiinflammatorische, antiatherogene und kardioprotektive Wirkung aufweist, während HMW Adiponektin eher proinflammatorische Wirkungen hat. Bei Patienten mit chronischer Nierenerkrankung (chronic kidney disease; CKD) werden erhöhte Gesamt-Adiponektin Konzentrationen gemessen. Ein spezifischer Anstieg einer einzelnen Subfraktion konnte bisher nicht festgestellt werden. Wir postulieren, dass bei eingeschränkter Nierenfunktion HMW Adiponektin selektiv erhöht ist, und überprüften die prädiktive Bedeutung der Adiponektinsubfraktionen für die Inzidenz von kardiovaskulären Ereignissen bei CKD Patienten.

Methodik: In die CARE FOR HOMe Studie wurden im Zeitraum von 2008 bis 2011 insgesamt 341 CKD G2 - G4 Patienten rekrutiert. Mit Hilfe eines standardisierten Fragebogens, körperlicher Untersuchung und Routinelabormethoden ermittelten wir das kardiovaskuläre Risikoprofil. Die Bestimmung von Gesamt- Adiponektin und seinen Subfraktionen erfolgte mittels kommerziell verfügbaren ELISA (ALPCO 6/1/2010, Salem, NH, USA). Zur Erfassung von kardiovaskulären Ereignissen mit dem primärem kombinierten Endpunkt ",atherosklerotisches Ereignis (inklusive Tod jedweder Genese)" und dem sekundären Endpunkt „akute kardiale Dekompensation (inklusive Tod jedweder Genese)" erfolgte jährlich eine Nachuntersuchung der Studienteilnehmer.

Ergebnisse: Erwartungsgemäß zeigt sich eine negative Korrelation von Gesamt Adiponektin mit der eGFR $(r=-0,145 ; p<0,001)$ und mit Body Mass Index (BMl; $r=-0,169 ; \mathrm{p}<0,001)$; diese betrifft alle Subfraktionen LMW (eGFR: $r=-0,148 ; p<0,001 ; B M I: r=-0,111 ; p=0,002)$, MMW (eGFR: $r=-0,154 ; p<0,001 ; B M l: r=-0,120 ; p=0,001$ ) und HMW (eGFR: $r$ $=-0,118 ; p=0,001 ; B M I: r=-0,172 ; p<0,001)$. Weibliche Studienteilnehmerinnen $(9,69 \pm 6,29 \mu \mathrm{g} / \mathrm{ml})$ weisen höheres Gesamt-Adiponektin auf als männliche Teilnehmer $(8,37 \pm 5,20 \mu \mathrm{g} / \mathrm{ml})$. Während der Nachbeobachtung traten bei 107 Patienten atherosklerotische Ereignisse, bei 86 Patienten akute kardiale Dekompensationen auf. Hierbei prädizierten Gesamt-Adiponektin und seine Subfraktionen atherosklerotische Ereignisse nicht. Hingegen waren höheres Gesamt-Adiponektin ebenso wie höheres HMW, MMW und LMW Adiponektin sowohl in univariaten als auch in multivarialen Cox-Regressionsanalysen Prädiktoren für kardiale Dekompensationen.

Diskussion: Entgegen unserer Hypothese tritt bei CKD kein selektiver Anstieg von HMW Adiponektin auf, sondern ein Anstieg aller Subfraktionen. Trotz der präsumptiv kardioprotektiven Eigenschaften ist erhöhtes Plasma-Adiponektin unabhängiger Prädiktor für kardiale Dekompensationen bei CKD Patienten. 
YIA 5

Begrenzte Bereitschaft zur Abklärung einer Hypercholesterinämie in einer Lipid-Spezial-Ambulanz: Ergebnisse einer Telefonumfrage an einem deutschen Universitätsklinikum

Sarah Zigan'; Prof. Dr. Michael Walter ${ }^{2}$

${ }^{1}$ Labor Berlin - Charité Vivantes $\mathrm{GmbH} ;{ }^{2}$ Labor Berlin - Charité Vivantes GmbH \& Institut für Laboratoriumsmedizin, Klinische Chemie und Pathobiochemie (Charité - Universitätsmedizin)

Hintergrund: Die Familiäre Hypercholesterinämie (FH; Häufigkeit: 1/250-500)) ist mit einem hohen kardiovaskulären Risiko und frühem Herztod assoziiert. Die Krankheit ist weltweit deutlich unter-diagnostiziert. Andererseits kann nur die frühe Diagnose und Behandlung mit Statinen das kardiovaskuläre Risiko reduzieren.

Ziel: An Hand des Surrogat-Markers LDL-Cholesterin sollten Patienten mit Verdacht auf FH erfasst und die weitere Strategie der behandelnden Teams erfragt werden.

Methoden: Über einen Zeitraum von 6 Monaten wurden alle LDL-Cholesterin-Messungen eines Universitätsklinikums mit LDL-Cholesterin-Konzentrationen $>190 \mathrm{mg} / \mathrm{dl}$ im Laborinformationssystem des Labors erfasst. Am Folgetag der Messung wurden an Hand einer Telefonumfrage die behandelnden Ärzte und Pflegeteams zur weiteren Strategie bezüglich Diagnostik und Therapie befragt.

Ergebnisse: Von insgesamt 46.663 eingegangenen Proben mit angeforderten Lipid-Parametern konnten 1.532 Patienten (3.3\%) mit LDL-Cholesterin-Konzentrationen $>190 \mathrm{mg} / \mathrm{dl}$ identifiziert werden. Davon waren 24 Patienten <20 Jahre, 174 Patienten 21-40 Jahre, 632 Patienten 41-60 Jahre und 702 Patienten $>60$ Jahre alt. Die Patienten kamen aus folgenden Fachabteilungen: Stoffwechsel (24,7\%), Kardiologie (17,8\%), Neurologie (13,4\%), Innere Medizin (17,5\%), Psychiatrie $(11,1 \%)$, Chirurgie (2,7\%) und Sonstige (12,8\%). 25,7\% aller Patienten mit V. a. FH hatten als Akut-Diagnose Myokardinfarkt, Apoplex oder Akutes Koronarsyndrom. 6,5\% der Patienten hatten diese Diagnosen in der Anamnese. Am Folgetag wurde für 664 Patienten mit Verdacht auf FH der behandelnde Arzt (42\% der Fälle) oder die mit dem Patienten betreute verantwortliche Pflegekraft ( $58 \%$ der Fälle) telefonisch kontaktiert. Hierbei konnte eruiert werden, dass $48,4 \%$ der Patienten zum Zeitpunkt der Befragung unter einer Lipid-senkenden Medikation standen. Unter keiner Lipid-senkenden Therapie standen 32,1\% der Patienten (unbekannt: 19,5\%). Von den Patienten, die nicht bereits schon in einer Stoffwechsel-Abteilung lagen, war bei 2,6\% der Patienten eine Überweisung zu einem Spezialisten in Planung; bei $73,7 \%$ der Patienten wurde diese als nicht notwendig angesehen und die Therapie entweder selbst durchgeführt $(64,4 \%)$ oder im Arztbrief angewiesen (9,1\%); keine Kenntnis oder Auskunft verweigert: $23,7 \%$ der Fälle.

Schlussfolgerung: Die Bereitschaft zur Abklärung einer ausgeprägten Hypercholesterinämie in einer Spezial-Ambulanz ist auch in einem Universitätsklinikum begrenzt. Die Untersuchungen deuten darauf hin, dass nicht bei allen Patienten mit Verdacht auf FH eine Leitlinien-gerechte Abklärung, Therapie und ggf. Familienuntersuchung erfolgt. Die Aussagekraft der Studie wird eingeschränkt durch den relativ hohen Anteil von Patienten mit Akut-Ereignissen und die schlechte telefonische Erreichbarkeit einiger Stationen. Auch die Ursache für den hohen Anteil von psychiatrischen Patienten mit Hypercholesterinämie (mit der Möglichkeit sekundärer Effekte) muss weiter abgeklärt werden.
YIA 6

Rezidivierende Infektionen bei rheumatoider Arthritis unter Immunsuppression

Dr. med. Stephan Thrum; Christoph GO Baerwald; PD Dr. med. habil. Olga Malysheva

${ }^{1}$ Universitätsklinikum Leipzig

Hintergrund: Für die Therapie von Autoimmunerkrankungen steht eine wachsende Auswahl immunsupprimierender Substanzen zur Verfügung, während langfristige Auswirkungen auf Immunsystem und Infektanfälligkeit sowie das Management hiermit bisher nicht ausreichend bekannt sind.

Fallvorstellung: Bei einer heute 61-jährige Patientin wurde vor 13 Jahren eine rheumatoide Arthritis mit klassischem Befallsmuster und positivem Antikörperbefund (Rheumafaktor, CCP-Antikörper) diagnostiziert. Im Verlauf der Erkrankung erhielt sie neben Glukokortikoiden verschiedene konventionelle DMARDs (MTX, Cyclosporin, Sulfasalazin) welche später bei Ineffektivität abgesetzt wurden. Seit einer Therapie mit Leflunomid 2010 entwickelten sich bei der Patientin rezidivierende Hautinfektionen mit Abszessbildung, deren Entstehung sich unter der späteren Therapie mit biologischen DMARD (Abatacept, Adalimumab) verstärkte. Eine erhöhte Infektneigung spiegelte sich auch im Auftreten einer Pneumonie und eines Herpes zoster. Hautinfektionen bestanden bereits in ihrer Kindheit, waren jedoch über Jahrzehnte nicht mehr aufgetreten. Bei schließlich nachgewiesenem Immunglobulinmangel werden seit 2014 Immunglobuline substituiert, worunter sich die Bildung weiterer interventionspflichter Hautinfektionen verhindern lässt. Daneben erhält die Patientin zum Remissionserhalt Prednisolon und Etanercept.

Schlussfolgerung: Das Wechselspiel zwischen Autoimmunität, Infektionen und Immundefekten ist komplex und Gegenstand aktueller Forschung. Eine Hypogammaglobulinämie stellt im klinisch-internistischen Alltag den behandelnden Arzt vor eine große diagnostische und therapeutische Aufgabe. So kann sie, wie in diesem Fallbericht, auf dem Boden eines primären Immundefektes bestehen oder sekundäre Auslöser haben. Viele Patienten mit einer CVID weisen Autoimmunerkrankungen auf. Gleichzeitig steigt die Prävalenz einer sekundären Hypogammaglobulinämie im Verlauf der letzten Jahrzehnte auch iatrogen durch den zunehmenden Einsatz von Immunsuppressiva. Daneben müssen hämatologische, renale und gastrointestinale Ursachen interdisziplinär geprüft und ausgeschlossen werden. Um die aufwendige und kostenintensive Therapie mit Immunglobulinen rational zu gestalten, ist daher ein gezieltes Vorgehen bei diesen Patienten notwendig. Eine entsprechende Diagnostik geht über die Kernaufgaben des Internisten hinaus und stellt ihn nicht zuletzt vor wirtschaftliche Probleme. Gleichzeitig fehlen derzeit Empfehlung und Leitlinien in diesem Bereich. Hier müssen weitere Erfahrungen gesammelt und zusammengefasst werden, um Handlungsempfehlungen abzuleiten. 


\section{Diabetologie}

\section{PS01}

Umstellung auf eine Basalinsulintherapie bei erwachsenen

Typ-2-Diabetes Patienten - Gemeinsame Auswertung

\section{der deutschen Diabetesregister DPV und DIVE}

Stefanie Lanzinger'; Prof. Dr. Thomas Danne; Prof. Dr. Reinhard Holl;

Prof. Dr. Jochen Seufert; Dr. med. Hans-Martin Reuter;

Dr. med. Stefan Sziegoleit; PD Dr. med. habil. Wolfram Karges;

Dr. med. Arthur Grünerbel; Dr. med. Dagmar Weise;

Prof. Dr. Peter Bramlage

1 Universität Ulm

Fragestellung: Welche Therapie erhalten erwachsene Typ-2-Diabetes ( $\left.\mathrm{T}_{2} \mathrm{D}\right)$ Patienten bevor eine Therapie mit Basalinsulin begonnen wird? Methoden: Aktuell sind 588.437 Diabetespatienten in DPV oder DIVE dokumentiert (Stand November 2016). Davon wurden $12.534 \mathrm{er}-$ wachsene (?18 Jahre) T2D Patienten mit Basalinsulingabe und Information zur Vortherapie untersucht. Ausschließlich T2D Patienten mit einer pharmakologischen Vortherapie wurden in die Auswertung eingeschlossen. Als Untersuchungszeitraum wurde der Beginn der Basalinsulingabe und 180 Tage zuvor betrachtet. Die Patienten wurden in 3 Therapiegruppen eingeteilt: 1) Umstellung von oralen Antidiabetika (OAD (ohne GLP-1-Analoga oder DPP4 Hemmer)) auf eine Basalinsulingabe, 2) Umstellung von einer Therapie mit GLP-1-Analoga oder DPP4 Hemmer (GLP1-DPP4) auf eine Basalinsulingabe, 3) Umstellung von einer supplementären Insulintherapie (SIT) auf eine Basalinsulingabe. Untersucht wurde, ob sich die Vortherapie in Bezug auf das Alter ( $<60$ Jahre, ?60 Jahre), Body-Mass-Index (BMI, <30 $\mathrm{kg} / \mathrm{m2}$, ?30 kg/m2) und in Bezug auf das Jahr der Therapieumstellung (vor 2014, 2014-2016) unterscheidet. Ergebnisse: Die Mehrheit (69,9\%) der T2D Patienten wurde von einer OAD-Therapie auf eine Basalinsulingabe umgestellt, während $18,9 \%$ von GLP-1-DPP4 und $11,2 \%$ von SIT auf eine Basalinsulingabe wechselten. Signifikante Unterschiede in der Vortherapie zeigten sich in Bezug auf das Alter und das Jahr der Therapieumstellung. Im Vergleich zur älteren Gruppe wurden T2D Patienten $<60$ Jahre weniger von $\mathrm{OAD}$, aber häufiger von GLP-1-DPP4 auf eine Basalinsulingabe eingestellt. Bei der Umstellung von SIT auf eine Basalinsulingabe zeigten sich keine signifikanten Unterschiede zwischen den Altersgruppen. Vor 2014 wurden T2D Patienten signifikant häufiger von OAD oder SIT auf eine Basalinsulingabe umgestellt, während von 2014-2016 der Anteil einer Umstellung von GLP-1-DPP4 auf eine Basalinsulingabe höher war. Schlussfolgerung: Die Ergebnisse der Diabetesregister DPV und DIVE zeigten einen Unterschied in der pharmakologischen Vortherapie vor Beginn einer Basalinsulingabe bei erwachsenen $T_{2} D$ Patienten. Unterschiede wurden insbesondere in Bezug auf das Alter der Patienten und auf das Jahr der Therapieumstellung beobachtet. Die Ergebnisse weisen auf die Wichtigkeit individueller Behandlungskonzepte sowie auf eine Veränderung der Wahl der Diabetestherapie hin.

\section{PS02}

Efficacy and Safety of Switching from Sitagliptin (SITA) to Liraglutide (LIRA) in Subjects with Type 2 Diabetes (T2D) Not Achieving Adequate Glycaemic Control on SITA and Metformin (MET): A Post Hoc Subgroup Analysis Defined by Baseline $B M I<$ or $? 30 \mathrm{~kg} / \mathrm{m} 2$

Dr. Marcel Kaiser'; Prof. Werner Kern²; Prof. Timothy S. Bailey ${ }^{3}$; Prof. Robert Takacs?; Prof. Paturi V. Rao?; Prof. George M. Tsoukas?; Dr. Markus Rieck?; Dr. Sidsel B. Christensen?; Dr. Margit S. Kaltoft?; Prof. Maximo Maislos?

${ }^{1}$ Diabetologische Schwerpunktpraxis, Frankfurt am Main; ${ }^{2}$ Zentrum für Hormon- und Stoffwechselerkrankungen,MVZ ENDOKRINOLOGIKUM UIm; ${ }^{3}$ AMCR Institute; ${ }^{4}$ University of Szeged; ${ }^{5}$ Ramdevrao Hospital, Hyderabad; ${ }^{6}$ McGill University Health Centre; ${ }^{7}$ Novo Norsisk Pharma GmbH; ${ }^{8}$ Novo Nordisk A $/ \mathrm{S} ;{ }^{9}$ Soroka UMC, Ben-Gurion University FOHS

Introduction: This post-hoc subgroup analysis of the multinational, randomised, double-blind, double-dummy Lira-SWITCH trial compared 26-week efficacy and safety of switching from SITA (treatment for ?9o days) to LIRA (both +MET), in subjects with $\mathrm{T}_{2} \mathrm{D}$ not achieving adequate glycaemic control on SITA+MET, according to baseline body mass index (BMI) (< or ?30 kg/m2).

Methodology: Subjects were randomised 1:1 to switch to LIRA 1.8mg $(n=203)$ or continue SITA 10omg/day $(n=204)$, both + MET.

Findings: At week 26 , subjects treated with LIRA vs. SITA achieved reduced $\mathrm{HbA}_{1 \mathrm{c}}$ (estimated treatment difference [ETD] -0.66\%; $\mathrm{p}=0.0001$ ), body weight (ETD -1.24 kg; $\mathrm{p}=0.0221$ ) and fasting plasma glucose (FPG) (ETD $-0.88 \mathrm{mmol} / \mathrm{L} ; \mathrm{p}=0.0058$ ) in the $B M \mathrm{C}<30$ subgroup. Reduced $\mathrm{HbA}_{1 \mathrm{c}}$ (ETD -0.58\% [ $\left.\mathrm{p}<0.0001\right]$ ), body weight (ETD $-1.71 \mathrm{~kg}$ $[p=0.0002)$ and FPG (ETD $-1.27 \mathrm{mmol} / \mathrm{L}[\mathrm{p}<0.0001])$ were also observed in the BMI?30 subgroup, together with systolic blood pressure (SBP) (ETD $-3.38 \mathrm{mmHg}[\mathrm{p}=0.0370])$. There was no treatment difference in $S B P$ in the $B M l<30$ subgroup $(p=0.8819)$. There were no significant differences in ETDs between BMl subgroups for all parameters ( $p>0.05$ ) except FPG $(p=0.024)$. With LIRA vs. SITA, adverse events (AEs) occurred in $69.0 \%$ vs. $52.6 \%$ of subjects in the BMl<30 subgroup, and $68.7 \%$ vs. $59.5 \%$ in the BMI?30 subgroup; serious AEs: $3.4 \%$ vs. $2.6 \%$ in the $B M l<30$ subgroup, and $2.6 \%$ vs. $4.0 \%$ in the BMI?30 subgroup. Confirmed hypoglycaemia occurred three times, all in subjects with BMI?30, treated with SITA.

Conclusion: Switching to LIRA resulted in superior $\mathrm{HbAlc}_{1}$, body weight and FPG reductions, compared with continued SITA treatment, irrespective of baseline BMI subgroup.

\section{PS03}

Health Status Assessed with Eq-5D In People With Type 2

Diabetes Participating In The Leader Trial

Prof. Dr. med. Johannes Mann'; Prof. Michael Nauck²; Dr. Jörg Lüdemann³ Prof. Staphan Jacob?; Dr. Markus Rieck?; Dr. Nana Kragh?;

Dr. Heidrun Bosch-Traberg,?; Prof. Stuart Pocock?

${ }^{1}$ Friedrich Alexander University of Erlangen; ${ }^{2}$ St. Josef-Hospital, Ruhr-University Bochum; ${ }^{3}$ diabetes-falkensee.de, Diabetes- und Fußzentrum, $;{ }^{4}$ Praxis für Prävention und Therapie; ${ }^{5}$ Novo Nordisk Pharma GmbH; ${ }^{6}$ Novo Nordisk A $/ S_{;}{ }^{7}$ London School of Hygiene \& Tropical Medicine

Objectives: The LEADER trial (Liraglutide Effect and Action in Diabetes: Evaluation of cardiovascular outcome Results) has reported cardiovascular risk reduction compared to placebo on top of standard of care (SoC), with a $13 \%$ risk reduction for CV death, $\mathrm{Ml}$ or stroke $(\mathrm{HR}=0.87$, $95 \% \mathrm{Cl}(0.78-0.97) \mathrm{p}=0.011)$. To understand the impact on patients' health status, EQ-5D-3L (5 dimensions: mobility, self-care, usual activities, pain/discomfort and anxiety/depression) was assessed. Patients also rated current health state on a vertical visual analogue scale (VAS). 
METHODS: LEADER was a long-term, multi-centre, international, randomised, double-blind, placebo-controlled cardiovascular outcomes trial assessing the safety of liraglutide $1.8 \mathrm{mg} / \mathrm{d} \mathrm{s.c.} \mathrm{vs} \mathrm{placebo} \mathrm{in} \mathrm{high}$ risk type 2 diabetes patients for up to 5 years. EQ-5D was measured in a sub-set of subjects $(n=2,960)$, i.e. all subjects from Canada, Denmark, Ireland, Italy, Netherlands, Germany, Spain, Sweden, UK and USA.

RESULTS: Baseline mean index score was 0.8 for both arms. Baseline VAS score was 74.5 ( 74.7 for liraglutide and 74.2 for placebo). By 3 years, deterioration in EQ-5D index-score was significantly less in the liraglutide group (-0.027) than in the placebo group (-0.046)(Estimated treatment difference (ETD) $0.01895 \% \mathrm{Cl} 0.001 ; 0.035) \mathrm{p}=0.034$ ). Decrease in EQ-5D VAS score was significantly less in the liraglutide group (-0.146) vs the placebo group (-1.449)(ETD 1.302 95\% Cl 0.101; 2.504; $p=0.034)$.

CONCLUSIONS: LEADER is the first trial in $\mathrm{T}_{2} \mathrm{D}$ to report a significant benefit in patient-reported health status when comparing an anti-hyperglycaemic treatment to SoC. Liraglutide improves health status by significantly decelerating the detrimental impact of complications of $\mathrm{T}_{2} \mathrm{D}$.

\section{PS04 \\ Liraglutide and Renal Outcomes in Type 2 Diabetes: Results of the LEADER trial}

Prof. Dr. med. Johannes Mann'; Prof. Michael Nauck²; Prof. Stephan Jacob; Dr. Jörg Lüdemann?; Dr. Kirstine Brown-Frandsen?; Dr. Markus Rieck?; Prof. Gilbert H Daniels?; Peter Kristensen?; Prof. Steven E Nissen?; Prof. Stuart Pocock?; Prof. Neil R Poulter'?; Dr. Lasse S Ravn?; Dr. Søren Rasmussen?; Prof. William M Steinberg ${ }^{11}$; Dr. Mette Stockner?; Prof. Bernard Zinman ${ }^{12}$; Prof. Richard M Bergenstal ${ }^{13}$;

Dr. Florian MM Baeres?; Prof. Steve P Marso'?; Prof. John B Buse'?

${ }^{1}$ Friedrich Alexander University of Erlangen; ${ }^{2}$ St. Josef-Hospital, Ruhr-University Bochum; ${ }^{3}$ Praxis für Prävention und Therapie; ${ }^{4}$ diabetes-falkensee. de, Diabetes- und Fußzentrum, ${ }^{5}$ Novo Nordisk A $/ S_{;}{ }^{6}$ Novo Nordisk Pharma $\mathrm{GmbH} ;{ }^{7}$ Massachusetts General Hospital; ${ }^{8}$ Department of Cardiovascular Medicine at Cleveland Clinic; ${ }^{9}$ London School of Hygiene \& Tropical Medicine; ${ }^{10}$ Imperial College London; ${ }^{11}$ George Washington University Medical Center; ${ }^{12}$ Mount Sinai Hospital; ${ }^{13}$ International Diabetes Center; ${ }^{14}$ University of Texas Southwestern; ${ }^{15}$ School of Medicine Chapel Hill

The effects of liraglutide, a long-acting glucagon-like peptide-1 (GLP1) analog, on renal outcomes in type 2 diabetes are unknown. We conducted a randomized, double-blind, placebo-controlled trial comparing liraglutide vs placebo, both in addition to standard of care,in participants with type 2 diabetes and high cardiovascular risk. The Liraglutide Effect and Action in Diabetes: Evaluation of cardiovascular outcome Results (LEADER) trial was initiated in 2010 and completed in 2016. Renal events were key secondary outcomes. The primary renal outcome was a composite of new onset of persistent macroalbuminuria, persistent doubling of serum creatinine, need for continuous renal replacement therapy, or death due to renal disease. Risk of renal outcomes was determined using intention-to-treat in time-to-event analyses; competing risk of death was taken into account. Loss of eGFR by $>-30 \%$ and change in eGFR was also analyzed. 9340 patients were randomized and median follow-up was 3.84 years. The primary renal outcome occurred in fewer participants treated with liraglutide (268 of 4668) than with placebo (337 of 4672; HR 0.787 [0.670;0.924] $\mathrm{p}=0.003)$. The difference was primarily driven by the findings for new onset of persistent macroalbuminuria, occurring in fewer participants treated with liraglutide (161 of 4668) than with placebo (215 of 4672; HR $0.74[0.61 ; 0.91] p=0.004)$. After an initial drop in both groups, eGFR decreased significantly less with liraglutide than placebo. There was no difference in participants having $>30 \%$ drop in eGFR between the two treatment groups. In two subgroups at renal risk, namely those with eGFR $<60 \mathrm{ml} / \mathrm{min}$ at baseline $(\mathrm{N}=2158)$ and with microalbuminuria at baseline $(\mathrm{N}=2458)$, similar, albeit nonsignificant, results were ob- served. In conclusion, liraglutide on top of standard therapy blunted progression of diabetic nephropathy.

\section{PS05}

Problematic drinking in the elderly and its association to muscle mass and muscle function in type II diabetes Dr. Nikolaus Buchmann; Dr. Dominik Spira; PD Dr. Kristina Norman; PD Dr. Ilja Demuth; Prof. Dr. med. Elisabeth Steinhagen-Thiessen Charité - Universitätsmedizin Berlin

Background: Alcohol consumption is particular serious in diabetic subjects $\left(T_{2} D\right)$, as hypoglycemic states can occur due to an inhibition of hepatic gluconeogenesis. Muscle is supplied with energy by the breakdown of glucose, frequent episodes of hypoglycemic conditions could result in muscle breakdown. Aim of the current analysis within the Berlin Aging Study II (BASE-II) was to analyze the association of problematic drinking behavior to muscle mass and muscle function in diabetes. Methods: Cross-sectional data of 1456 elderly BASE-II participants ( $50.8 \%$ women; $64 \pm 4$ years old) were analyzed. Alcohol consumption and problematic drinking was assessed using the Alcohol Use Identification Test (AUDIT). Muscle mass was measured using dual energy X-ray absorptiometry (DXA), grip strength using a Smedley dynamometer. Adjusted regression models were calculated to assess the association of problematic drinking to muscle mass and grip strength. Results: Problematic drinking was evident in $11.1 \%$ of BASE-II participants, $12.1 \%$ had diabetes. In adjusted models (adjustment for age, BMI, smoking status, morbidities, HOMA-IR, CRP, TSH, physical activity and antidiabetic medication) we found a statistically significant association between problematic drinking to muscle mass (Beta: -22.5; SE: 9.8; $p=0.026$ ) and grip strength (Beta: -7.8; SE: 2.9; $p=0.010$ ) in elderly diabetic men. These results were not evident in women and subjects without $T_{2} D$. Discussion: Alcohol consumption, respectively problematic drinking is associated with poorer muscle mass and grip strength in elderly men with diabetes. This topic should be addressed in these subjects as they could be at increased risk for early dependency.

\section{Digitale Medizin / Palliativmedizin / Genderspezifische Aspekte der Inneren Medizin}

\section{PS06}

\section{Elektronisches Anordnen mit Anordnungssets}

Susanne Decker; Laura Zwack; Dr. Michael von Wagner; Guido Garlip; Walid Sbaih; Dr. Martin Kuhrau; Stephan Essmeyer

Elsevier Gmbh

Background: Elektronische Anordnungssets können den Prozess des Anordnens beschleunigen, die Zufriedenheit der Ärzte steigern und die Verweildauer reduzieren, so die Ergebnisse von zwei Pilotprojekten am Universitätsklinikum Frankfurt und der LVR-Klinik Langenfeld.

Einleitung: In den USA nutzen bereits über $70 \%$ aller Krankenhäuser Anordnungssets in ihrem KIS. Anordnungssets sind vorgefertigte Auswahllisten von Anordnungen für eine spezifische Diagnose oder Prozedur. Sie reflektieren den aktuellen Stand der Leitlinien und Evidenz und werden ins Klinikinformationssystem (KIS) integriert. Ein Anordnungsset umfasst dabei alle Anordnungen, die für die jeweilige Diagnose oder Prozedur vom Arzt gemacht werden müssen. Auch in Deutschland wird immer häufiger elektronisch über das KIS angeordnet. Anwender bemängeln jedoch dabei Redundanzen im Prozess und verschachtelte Menüs. Sie wünschen sich Arbeitserleichterungen z.B. in Form von Anordnungssets. Als weltweit führender Wissen- 
schaftsverlag wollen wir einen Beitrag zur strukturellen und inhaltlichen Verbesserung des elektronischen Anordnens leisten.

Material und Methoden: Ende 2015/ Anfang 2016 startete die Pilotierung. Es wurden fünf Anordnungssets in das ORBIS KIS von Agfa Healthcare am Uniklinikum Frankfurt und drei Anordnungssets in NEXUS Case Maps der LVR-Klinik Langenfeld integriert. Anschließend wurde der Einfluss auf die Geschwindigkeit des Anordnens, die Zufriedenheit der Ärzte mit dem Prozess und der Einfluss auf die mittlere Verweildauer evaluiert.

Ergebnisse: Auf alle drei Evaluationsaspekte bezogen, konnten Verbesserungen erzielt werden. Am Universitätsklinikum Frankfurt führte das elektronische Anordnen im KIS zu einer Reduktion der mittleren Verweildauer von 8,53 Tagen auf 8,05 im Beobachtungszeitraum. Eine vergleichende Zeitmessung von 27 Patientenfällen ohne Anordnungsset versus 27 virtuellen Patientenfällen mit Anordnungssets bewirkte eine Beschleunigung des Prozesses um 42 Sekunden pro Patient festgestellt. Online-Befragungen unter 22 anordnenden Ärzten der beiden Kliniken in den Pilotabteilungen vor und nach Einsatz der Anordnungssets zeigen auch eine deutliche Verbesserung der Zufriedenheit mit der inhaltlichen Unterstützung des Anordnungsprozesses durch Anordnungssets.

Ausblick: Beide Pilotpartner hat die Anwendung der Anordnungssets so überzeugt, dass die Nutzung fortgesetzt wird. Im nächsten Schritt arbeiten fünf Kliniken gemeinsam mit Elsevier an der Entwicklung eines standardisierten Meta-Katalogs der anordnenbaren Leistungen. So können Anordnungssets in Zukunft in einem standardisierten Format und in einer standardisierten Sprache entwickelt werden, die einen einfachen Import und Export ins KIS ermöglichen.

\section{PS07}

Interactive algorithm for the measurement of pancreatic cancer liver metastases in ultrasound

Dr. med. Alexander Hann'; Dieter Schmalstieg ${ }^{2}$; Xiaojun Chen ${ }^{3}$, Wolfram Zoller'; Jan Egger ${ }^{2,5}$

1 Department of Internal Medicine I, Ulm University, Ulm, Germany; ${ }^{2}$ Institute for Computer Graphics and Vision, Graz University of Technology, , Graz, Austria; ${ }^{3}$ Shanghai Jiao Tong University, School of Mechanical Engineering, Dong Chuan Road, Shanghai, China. ${ }^{4}$ Department of Internal Medicine and Gastroenterology, Katharinenhospital, Stuttgart, Germany, ${ }^{5}$ BioTechMed, Krenngasse, Graz, Austria.

Background: Most patients with pancreatic adenocarcinoma (PDAC) are diagnosed in a palliative state with liver metastases. New life prolonging first line chemotherapies like FOLFIRINOX or Gemcitabine/ nab-Paclitaxel and second line therapies like OFF or Nanoliposomal Irinotecan/5FU are prolonging the time during treatment. Ultrasound (US) is one of the most cost effective form of staging these patients. Unfortunately due to a high interobserver bias and the low image quality patients often receive alternative staging methods. In this work we developed an interactive computer based algorithm for recognition of liver metastases on US B-mode images and evaluated it on a set of clinical images.

Methods: The algorithm was initially trained using liver metastases with different echogenic appearances. In the second step we evaluated the method on images including liver metastases of patients with PDAC. We compared the results against manual ground truth segmentations.

Results: In total 40 clinical images have been evaluated with our method. The results yielded an average Dice Score of $85 \%$ and an average Hausdorff Distance of 13 pixels. Conclusion: This new interactive real-time behavior approach can help clinicians to perform fast and accurate US staging of pancreatic cancer liver metastases.

\section{PS08}

Hämorrhagische Diathese als Leitsymptom eines metastasierten Adenokarzinoms der Lunge

Dr. med. Anke Cornelia Spoo'; Dr. med. Friedemann Köhler; Prof. Dr. Paul La Rosee

${ }^{1}$ Schwarzwald-Baar Klinikum Villingen-Schwenningen

Einleitung: Eine spontan auftretende hämorrhagische Diathese stellt eine vitale Bedrohung für den Patienten und eine komplexe diagnostische Herausforderung für den Kliniker dar.

Fallbericht: Ein 63-jähriger Patient wurde aufgrund einer akut manifesten hämorrhagischen Diathese mit Spontanhämatomen und einer Makrohämaturie zur Diagnostik stationär aufgenommen. Labordiagnostisch auffällig waren eine erniedrigte Prothrombinzeit, eine nicht messbare aktivierte partielle Thromboplastinzeit, eine Hypofibrinogenämie bei synchron erhöhten D-Dimeren und ein Faktor XIII-Mangel. Antithrombin, Thrombinzeit, Thrombozytenzahl und die Aktivität der Gerinnungsfaktoren II, V, IX, XI und XII lagen im Referenzbereich. Hinweise auf eine Hemmkörperhämophilie oder ein Phospholipid-Syndrom ergaben sich nicht. Das Blutbild mit Differentialblutbild zeigte keine signifikant pathologische Werte. Serochemisch auffällig waren eine erhöhte LDH ohne Zeichen einer intravasalen Hämolyse und ein erhöhter CEA-Wert. In der körperlichen Untersuchung zeigten sich bis auf multilokulär fassbare Suffusionen keine Auf-fälligkeiten. In der Ganzkörper-CT-Untersuchung wurde eine Raumforderung von 1,9 × 3,1 cm im linken pulmonalen Oberlappen mit lymphonodulärer, pulmonaler und ossärer Metastasierung detektiert. Ein tumoröses Geschehen im Bereich des Urogenital- und des oberen und unteren Gastrointestinaltraktes wurde endoskopisch ausgeschlossen. Bei hohem interventionellem Risiko einer endoluminalen bronchopulmonalen und mediastinalen Diagnostik erfolgte unter Substitutionstherapie mit Gerinnungsfaktoren und antifibrinolytischer Therapie eine Knochenmarkpunktion. Die multimodale Aufarbeitung des Aspirates ergab ein TTF-1 positives Adenokarzinom ohne molekulare Treibermutationen (EGFR-; ROS-; ALK-). Eine Chemotherapie mit Carboplatin (AUC5)/Paclitaxel 175 mg/m2 KO wurde eingeleitet. Darunter normalisierten sich die auffällig beschriebenen Gerinnungsparameter und die Blutungsneigung noch vor dem bildgebend dokumentierten Tumoransprechen. Diskussion: Eine Knochenmarkkarzinose mit hämorrhagischer Diathese aufgrund einer disseminierten intravasalen Koagulation (DIC) findet sich am häufigsten bei Karzinomen der Prostata und der Mamma. Bei Lungenkarzinomen ist eine Knochenmarkkarzinose als Erstmanifestation mit schwerer hämorrhagischer Diathese eine bisher nicht beschriebene Rarität. Auffällig ist in diesem Fall das diagnostisch nicht richtungsweisende Differentialblutbild ohne Leukoerythroblastose. Durch eine Knochenmarkpunktion war es möglich, minimalinvasiv die Histopathogenese der Tumor-erkrankung sowie die Ursache der plasmatischen Gerinnungsstörung im Sinne einer sekundären Hyperfibrinolyse aufzuklären. Die rasch eingeleitete antineoplastische Systemtherapie konnte die Blutungsneigung effektiv und anhaltend durchbrechen. 
PS09

Modernes interdisziplinäres Management einer uretero-iliakalen Fistel (Fallbericht)

Dr. med. Diana Benedix; Prof. Dr. Frank Meyer;

PD Dr. med. habil. Andrej Udelnow; Prof. Dr. med. Frank Fischbach;

Prof. Dr. med. Zuhir Halloul

Universitätsklinikum Magdeburg A.ö.R.

Einleitung: Außergewöhnliche Kurzschlussverbindungen des arteriellen Systems zu luminalen Organsystemen stellen eine Herausforderung in ihrem adäquaten diagnostischen und therapeutischen Management dar. Ziel: Wissenschaftlicher Fallbericht mit kasuistischer Darstellung der erfolgreichen, kombiniert gefäßchirurgisch und interventionell-radiologischen Versorgung einer seltenen rechtsseitigen uretero-iliakalen Fistel, basierend auf gewonnenen klinischen Erfahrungen, einer selektiven Literaturrecherche sowie der Diskussion empfohlener Maßnahmen des Vorgehens in Diagnosefindung und abgeleiteten Therapien.

Fallkonstellation: Stationäre Aufnahme eines 79-jährigen Patienten mit Hämaturie und Blasentamponade bei Z.n. katheterversorgten, aktinisch bedingten Ureterstrikturen beidseits infolge neoadjuvanter Radiochemotherapie eines späterhin mittels abdominoperinealer Rektumexstirpation operativ versorgten supraspinctären Karzinoms (урTзурNoMo). Die Laborkontrolle erbrachte eine Anämie, die transabdominale Sonografie eine Harnstauung bds. Die komplementäre $\mathrm{CT}$ wies keine manifeste Blutung infolge inkonsistenter Hämorrhagie nach.

Interventionelle Maßnahmen: Zunächst erfolgten die Blasenspülung und der ureterale Katheterwechsel. Aufgrund der ausgelösten rechtsseitigen endoluminal-ureteralen Blutung wurde trotz nicht gegebenem CT-basierten Nachweis der Blutungslokalisation (jedoch Bestätigung von Blasentamponade und Nierenstauung rechts, aber akuter Blutung aus dem rechten Ureterenostium in der Zystoskopie) die kombiniert gefäßchirurgische (arterieller Zugang rechts-femoral, abschließend notwendige Gefäßdesobliteration und Intimarefixation in $\operatorname{der}$ A. femoralis superficialis dextra infolge manipulativ provozierter Dissektion) / interventionell-radiologische Versorgung (Amplat-

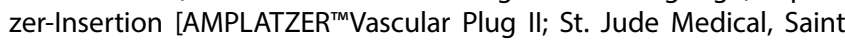
Paul, Minnesota,USA] in die A. iliaca interna dextra und rechts-iliacales Stenting durch "cross-over"-Manöver von links-femoral) vorgenommen.

Verlauf: Zeitgerechte Stabilisierung des Patienten mit periinterventioneller ITS-Betreuung sowie Entlassung am 15. postinterventionellen Tag ohne Anhalt für lokales Rektumkarzinomrezidiv und Re-Blutung bei weiterhin erforderlichen ureteralen J-Katheterwechseln. Zusammenfassung: Die zügig zu initiierende (gefäßchirurgische und interventionell-radiologische) Hybrid-Operation ist im präsentierten Fall als absolut indizierte und prognostisch aussichtsreiche Vorgehensweise für die rezidivierende arterielle Blutung mit klinischer Manifestation der Hämaturie und Blasentamponade einer uretero-iliacalen Fistel anzusehen.

Schlussfolgerung: Das minimal-invasive Herangehen mittels Stenting ist heute die Methode der Wahl im sequenziellen und notwendig zeitgerechten Management der potenziell lebensbedrohlichen uretero-iliacalen Fistel aufgrund einer arteriell bedingten endoluminalen Blutung und des drohenden hämorrhagischen Schocks.
PS10

Chirurgie von Vena-cava-inferior (VCI)-assoziierten Tumorläsionen - Überblick, Fallserie und Behandlungsergebnisse

Prof. Dr. med. Zuhir Halloul; Prof. Dr. Frank Meyer

Universitätsklinikum Magdeburg A.ö.R.

Hintergrund: Tumorläsionen der V. vava inferior (VCl) können sich von der Vene selbst oder von malignen Tumorinfiltrationen des umgebenden Gewebes entwickeln. Tumoren mit in die V. cava reichenden Tumorzapfen verdienen dabei noch eine besondere Beachtung. Das Ziel dieser außergewöhnlichen und repräsentativen Fallserie aus einem chirurgischen Zentrum war, die spezifische, individuell adaptierte operative Vorgehensweise und deren Ergebnisse, verbunden mit prognostischen Betrachtungen, zu analysieren.

Patienten und Methoden: In einem Zeitraum von 6 Jahren wurden alle Patienten mit Tumorläsionen der VCI mit patienten- und tumorspezifischen Merkmalen konsekutiv erfasst. Diagnostische Parameter und therapeutisches Management wurden dokumentiert und das kurz- bzw. langfristige "Outcome" (Komplikationsrate, perioperative Morbidität/Mortalität, Rezidivrate, Überleben) durch periodische „Follow- up"- Untersuchungen bestimmt.

Ergebnisse: Insgesamt wurden 12 Patienten in der Studie im Zeitraum vom 01.01.01-31.12.07 erfasst: 6 primäre VCl-Tumore (Leiomyosarkome) und 6 sekundäre VCl- Tumore ( 2 retroperitoneale Läsionen: 16,7 \%; 3 Nierenzellkarzinome: 25 \%; 1 Nebennierenzellkarzinom: 8,3\%). 4 der sekundären VCl-Tumore hatten Tumorzapfen in der VCl gebildet. Die Ro- Resektionsrate war $83 \%$. Die perioperative Morbidität betrug 33 $\%$, hingegen lag die Hospitalmortalität bei $8,3 \%(n=1)$. Die chirurgische Rekonstruktion der VCl wurde in allen Fällen erreicht (100\%). Der durchschnittliche postoperative Beobachtungszeitraum betrug 20 Monate (Spannbreite: 1-58 Monate). Die Follow-up-Dokumentation erfasste $100 \%$ der Patienten. 3 Patienten erlitten ein Tumorrezidiv $(27,5 \%$ von $n=11)$. Während die Gesamtmortalität während des Beobachtungszeitraumes $27,5 \%$ betrug, lag die Tumor-spezifische Mortalität bei $16 \%$. Schlussfolgerung: Das primäre chirurgische Ziel ist die Ro-Resektion des Tumors, um ein Langzeitüberleben ohne Tumorrezidiv einschließlich einer Rekonstruktion der VCI mit vernünftigem Nutzen-Risiko-Verhältnis zu erreichen. Die ansprechenden Ergebnisse dieser Fallserie zeigen, dass VCl-assoziierte Tumorläsionen chirurgisch therapierbar sind. Voraussetzungen hierzu sind die entsprechende Expertise des chirurgischen Teams, das suffiziente perioperative Management und ggf. Nutzung multimodaler Therapiekonzepte bei angemessener Überlebensrate. Die unterschiedliche Prognose der verschiedenen Tumorläsionen hängt von Tumorentität, Stadium, Resektionsstatus und individuellen Risikofaktoren ab.

\section{PS11}

Geschlechtsabhängige Unterschiede in der Behandlung

des Rektumkarzinoms im Rahmen einer prospektiven multizentrischen Beobachtungsstudie

Julia Katzenstein; Prof. Dr. Frank Meyer ${ }^{2}$; PD Dr. Henry Ptok; PD Dr. Ralf Steiner; Prof. Dr. Stephanie Wolff; Ronny Otto;

Prof. Dr. med. Hans Lippert; Prof. Dr. med. Ingo Gastinger

${ }^{1}$ AMEOS Klinikum Aschersleben; ${ }^{2}$ Universitätsklinikum Magdeburg A.ö.R.

Hintergrund: Gendermedizinische Aspekte sind zunehmend in die Betrachtung der klinischen Medizin gerückt, so auch in der Onkochirurgie. Ziel: Analyse geschlechterspezifischer Unterschiede im frühpostoperativen und onkologischen Outcome nach Rektumkarzinomresektion, basierend auf den Daten einer prospektiven multizentrischen Beobachtungsstudie.

Methode: Im Rahmen der multizentrischen prospektiven Observationsstudie "Qualitätssicherung Rektumkarzinom" wurden u.a. Daten 
zur Tumorlokalisation, exogenen und endogenen Risikofaktoren, neoadjuvanten Behandlung, Operationsverfahren, Tumorstadium, intra- und postoperativen Komplikationen von Patienten mit einem histologisch gesicherten Rektumkarzinom erfasst. Die Daten der Jahre 2005/2006 und 2010/2011 wurden hinsichtlich geschlechtsspezifischer Unterschiede in der postoperativen Morbidität, Hospital- und 30-Tage-Letalität, Lokalrezidivrate, im tumorfreien und Gesamt-Überleben univariat und multivariat analysiert. Ergebnisse: Insgesamt wurden 10.657 Patienten erfasst, 60,9\% waren männlich. Diese weisen in beiden Studienzeiträumen einen signifikant höheren Missbrauch von Alkohol $(P<0,001)$ und Nikotin $(P<0,001)$ auf und hatten einen tendenziell höheren BMI als Frauen. Bei nur tendenziellen Unterschieden der Tumorlokalisation wurde eine neoadjuvante Radiochemotherapie bei männlichen Patienten häufiger eingesetzt $(P<0,001)$. Außerdem wurden männliche Patienten häufiger einer abdominoperinealen Rektumexstirpation unterzogen $(\mathrm{P}<0,001)$. Die multivariate Analyse zeigte für männliche Patienten in beiden Zeiträumen eine höhere Gesamtmorbidität (OR: 1,5; $\mathrm{P}<0,001)$ und im Zeitraum 2010/2011 eine höhere Hospitalletalität (OR: 1,8; $\mathrm{P}<0,001)$. Bei einer medianen Nachbeobachtungszeit von 36 Monaten hatte das Geschlecht weder auf das Gesamtüberleben, das tumorfreie Überlaben noch auf die Lokalrezidivrate einen signifikanten Einfluss. Nach 5 Jahren lag das Gesamtüberleben über alle Stadien bei $60,5 \%$, das tumorfreie Überleben bei $63,8 \%$ und die Lokalrezidivrate bei $5 \%$.

Schlussfolgerung: Von anderen Einflussgrößen unabhängig bestanden Geschlechtsunterschiede nach operativer Behandlung von Rektumkarzinompatienten im früh-postoperativen Outcome, nicht jedoch hinsichtlich der onkologischen Langzeitergebnisse.

\section{PS12}

Assoziation von positiver Familienanamnese mit koronaren Vasomotionsstörungen im Acetylcholintest bei Frauen mit Angina pectoris und nicht-stenosierten Koronararterien PD Dr. Peter Ong; Dr. Giancarlo Pirozzolo; Dr. Anastasios Athanasiadis; Prof. Dr. med. Udo Sechtem

Robert-Bosch-Krankenhaus

Hintergrund: Patienten mit Angina pectoris und Verdacht auf eine stenosierende KHK weisen in ca. 50\% der Fälle keine relevanten Koronarstenosen auf. Ca. $60 \%$ dieser Patienten haben im Acetylcholintest einen auffälligen Befund im Sinne einer koronaren Vasomotionsstörung (epikardiale Spasmen bzw. koronare mikrovaskuläre Dysfunktion). Frauen sind von diesem Phänomen häufiger betroffen als Männer. Die Ursachen hierfür sind aber noch nicht geklärt. Genetische Polymorphismen für koronare Vasomotionsstörungen wurden an europäischen Patienten bislang nicht untersucht bzw. identifiziert Oft findet sich aber eine positive Familienanamnese bei diesen Patienten. Ziel dieser Studie ist es, zu untersuchen, in wie weit eine positive Familienanamnese für kardiovaskuläre Erkrankungen signifikant häufiger bei Patienten mit koronaren Vasomotionsstörungen auftritt.

Methoden: In einem Zeitraum von 3 Jahren wurden insgesamt 415 konsekutive Patienten (mittleres Alter $62 \pm 10$ Jahre, $62 \%$ Frauen) mit stabiler Angina pectoris und ohne relevante Koronarstenosen (Lumenreduktion $<50 \%$ ) einem Acetylcholintest unterzogen und in die Studie eingeschlossen. Dieser ergab bei 33\% der Patienten einen epikardialen Spasmus, bei $30 \%$ eine mikrovaskuläre Dysfunktion und bei $37 \%$ einen negativen Befund. Die Analyse der kardiovaskulären Risikofaktoren umfasste die arterielle Hypertonie, Hypercholesterinämie, Diabetes mellitus Typ II, Zigarettenrauchen sowie die Familienanamnese für kardiovaskuläre Erkrankungen definiert als ein Verwandter ersten Grades mit Myokardinfarkt oder Schlaganfall.

Ergebnisse: Ein pathologischer ACh-Test war häufiger bei Frauen als bei Männern zu finden ( $72 \%$ vs. $49 \%, p<0,0005)$. Frauen mit pa- thologischem $\mathrm{ACH}$-Test hatten signifikant mehr mikrovaskuläre als epikardiale Funktionsstörungen ( $57 \%$ vs. $43 \%, p<0,0005$ ). Männer mit pathologischem $\mathrm{ACH}$-Test dagegen mehr epikardiale als mikrovaskuläre Funktionsstörungen ( $74 \%$ vs. $26 \%$, p<0,0005). Eine positive Familienanamnese fand sich insgesamt bei $55 \%$ der Patienten und war häufiger bei Frauen als bei Männern zu finden ( $p=0,001,61 \%$ vs. 45\%). Unter den Patienten mit epikardialen Spasmen hatten Frauen auch signifikant häufiger eine positive Familienanamnese als Männer ( $p=0,006,66 \%$ vs. $42 \%)$.

Schlussfolgerung: Patienten mit Angina pectoris und nicht-stenosierten Koronararterien sind häufiger Frauen, die oft eine koronare Vasomotionsstörung im Acetylcholintest aufweisen. Epikardiale Spasmen bei Frauen sind mit einer positiven Familienanamnese assoziiert, was auf eine genetische Komponente dieser Erkrankung hindeutet.

\section{Endokrinologie}

\section{PS13}

Adipozytokine als Bindeglied zwischen Metabolismus und Arthrose

Marie-Lisa Hülser'; Carina Schreiyäck'; Yubin Luo ${ }^{2}$; Aline Bozec²; Georg Schett ${ }^{2}$; Prof. Dr. med. Ulf Müller-Ladner'; Elena Neumann ${ }^{1}$ 1 Justus-Liebig-Universität Gießen, Abteilung für Innere Medizin mit Schwerpunkt Rheumatologie, Kerckhoff-Klinik, Bad Nauheim, Deutschland; ${ }^{2}$ Universität Erlangen-Nürnberg, Abteilung Medizinische Klinik ${ }^{3}$ Rheumatologie und Immunologie, Erlangen, Deutschland

Hintergrund: Im Rahmen der Arthrose (OA) konnte u.a. durch unsere Arbeitsgruppe gezeigt werden, dass Adipokine immunmodulatorische Eigenschaften besitzen. Adipokine sind bei Adipositas und Insulinresistenz (häufige Komorbiditäten der Arthrose) von Bedeutung. Um die Zusammenhänge von Metabolismus und Arthrose über Adipokine zu untersuchen, wurden diese durch Kombination zweier Mausmodelle untersucht. Dabei wurde insbesondere auf die Unterscheidung zwischen systemischen und lokalen Einflüssen eingegangen.

Methodik: Bei Mäusen wurde eine Insulinresistenz durch high-fat diet (HFD) induziert bzw. die Kontrollgruppe mit normaler Diät (ND) gefüttert, gefolgt von chirurgischer OA-Induktion durch Destabilisierung des medialen Meniskus (DMM). Systemische Spiegel von Leptin, Adiponektin, Visfatin und IL-6 wurden mittels ELISA im Serum gemessen. Die murinen Gelenke wurden histologisch gefärbt (H/E-, Safranin-O-, Pappenheim- und Masson-Goldner Trichromfärbung), um den Arthrosegrad einzustufen und immunhistochemisch (Adiponektin, Leptin, Visfatin und F4/80) gefärbt um lokale Adipokinverteilungen darzustellen.

Ergebnisse: Bei allen Tieren war IL-6 systemisch nicht erhöht, sodass eine Entzündung, z.B. durch den Eingriff, ausgeschlossen wurde. Histologisch wurde eine OA in Tieren mit HFD stärker induziert und war zu verschiedenen Zeitpunkten sichtbar. Die HFD führte zu signifikant erhöhten Leptinwerten im Serum (z.B. ND 18.4; HFD 85.9ng/ml an Tag 28), während die anderen Adipokine wenig auf HDF reagierten. DMM führte zu reduzierten systemischen Leptinspiegeln (3/6 Gruppen signifikant) unabhängig von der Diät. Auffällig war, dass Adiponektin systemisch erst 8 Wochen nach OP erhöht war, dies jedoch unabhängig von der Diät.

Schlussfolgerung: Tiere mit Adipositas/Insulinresistenz haben einen schwereren Arthroseverlauf. DMM reduziert systemische Leptinwerte, wohingegen Leptin durch die HFD erhöht wird. Adiponektin wird erst im fortgeschrittenen Stadium der OA systemisch beeinflusst. 
Demnach scheinen selektiv Adipokine durch DMM/Arthrose zu unterschiedlichen Zeitpunkten reguliert zu werden.

\section{PS14 \\ Testosteron-Bestimmung bei Frauen - Klinische Klassifizierung beeinträchtigt durch die verwendete Messmethodik \\ Dr. med. Matthias Weber'; Prof. Dr. Manfred Rauh ${ }^{2}$ \\ ${ }^{1}$ MVZ Labordiagnostik Karlsruhe; ${ }^{2}$ Universitäts Erlangen, Kinder- und Jugendklinik}

Serum-Testosteron-Spiegel bei Frauen werden z.B. bestimmt für die Abklärung einer Virilisierung, Infertilität oder zum Nachweis hormonaktiver Tumoren. Seit langem sind die Unzulänglichkeiten immunologischer Steroid-Assays bekannt. Durch die inzwischen allgemeine Verfügbarkeit der Flüssig $\neg$ chromato $\neg$ grafie-Tandem $\neg$ massen $\neg$ spektro-metrie (LC-MS/MS) als hoch spezifischem und sensitivem Analyseverfahren sind deutliche Verbesserungen in der Assayqualität möglich geworden. Bei 980 Serumproben von weiblichen Individuen wurden die Testosteron-Ergebnisse des routinemäßig verwendeten automatisierten Ligandenbindungsassay (CLIA) verglichen mit den Ergebnissen einer LC-MS/MS (Inhaus-Verfahren). 30 Proben mit der größten Abweichung wurden mit einer zweiten LC-MS/MS-Methode nachgemessen. Bei beiden LC-MS/MS-Verfahren handelt es sich um Inhaus-Verfahren, die mit CRM mit zertifizierten Referenzmethodenwerten harmonisiert wurden. Die Klassifizierung der Ergebnisse erfolgte nach den jeweils etablierten Referenzintervallen (Cobas, Herstellerangaben, LCMS- Referenzwertstudien mit LCMS). Die Übereinstimmung der Messwerte zwischen Immunoassay und LC-MS/MS ergab einen mittleren Bias von $-11 \%$ bei einem Vertrauensbereich von $-114 \%$ bis $93 \%$ vom Mittelwert. Die Passing-Bablock-Regression ergab $\mathrm{CLIA}=\mathrm{LCMS} * 1.15$-0.19. In der klinischen Bewertung wurden im Immunoassay 163 Proben erhöht gemessen, von denen nur 63 Proben (39\%) gemäß der LC-MS/MS tatsächlich erhöht waren. Die Aussagekraft der Testosteronmessung mit dem Immunoassay ist bei Frauen also sehr begrenzt. Die Streuung um den Referenzmethodenwert ist erheblich und hat Auswirkungen auf die klinische Bewertung.

\section{PS15 \\ Hohe Rate von Fehlklassifizierung abhängig vom verwendeten Assay für die Vitamin D-Bestimmung Dr. med. Matthias Weber'; Prof. Dr. Manfred Rauh² \\ ${ }^{1}$ MVZ Labordiagnostik Karlsruhe; ${ }^{2}$ Universitätsklinikum Erlangen, Kinder- und Jugendklinik}

Im Rahmen eines Methodenvergleichs wurden in 197 unselektionierten Routine-Serumproben der Vitamin D-Spiegel mittels Immunoassay (Liaison, Diasorin) und LC-Tandem-MS bestimmt. Proben mit der höchsten Abweichung wurden in einem Referenzlabor ebenfalls mittels LC-Tandem-MS kontrolliert. Die Bewertung anhand klinischer Entscheidungsgrenzen wurde verglichen. Die im verwendeten Immunoassay gefundenen Spiegel lagen im Vergleich zum LC-MS-Wert systematisch 20\% niedriger. Der Streubereich im Bland-Altman-Plot betrug $-2 \%$ bis $+52 \%$. In der klinischen Klassifizierung fand sich bei ca. $30 \%$ aller Proben eine diskrepante Klassifizierung, unabhängig vom verwendeten Referenzbereich. Die mittels Immunoassay bestimmten Werte weichen systematisch und zufällig vom LC-MS-Wert ab. Insbesondere sind die von Patient zu Patient gefundenen Abweichungen deutlich größer, als die in Ringversuchen mit einzelnen Proben gefundenen Streuungen vermuten lassen würden. Die Verwendung eines Immunoassays zur Vitamin D -Bestimmung wird sowohl im Routinelabor, insbesondere aber zur Stratifizierung von Probanden im Rahmen von Studien als kritisch betrachtet. Auch mit Immunoassays erhobene Prävalenzstatistiken zur Vitamin D-Versorgung werden einen vom verwendeten Immunoassay abhängigen Bias aufweisen, der nicht unerheblich ist.

\section{PS16}

Multiple pathologische Frakturen und Hypophosphatämie infolge eines mesenchymalen Tumors der Plantaraponeurose Dr. med. Melanie Kandulski; Dr. Martin Röpke; Dr. Kirsten Reschke Universitätsklinikum Magedeburg

Eine 70jährige Patientin wurde uns zur Abklärung pathologischer Frakturen im Bereich des rechten Oberschenkels, beider Unterschenkel und Füße sowie Rippenserienfrakturen zugewiesen. Zuvor konnte mittels DEXA-Röntgen eine Osteopenie (rechter SH -1,8; LWS -1,5) gesichert werden und es wurde eine Substitution mit Cholecalciferol eingeleitet. Im initialen Labor waren eine Hypophosphatämie mit $0,62 \mathrm{mmol} / \mathrm{l}$ sowie eine verminderte tubuläre Phosphatreabsorption nachweisbar, so dass der Verdacht auf eine tumorinduzierte Osteomalazie (erworbener Phosphatdiabetes) gestellt wurde. In der Octreotide-Szintigrafie zeigte sich nur im Bereich des rechten Schilddrüsenlappens eine pathologische Radionuklidanreicherung. Nach einer rechtsseitigen Hemithyreoidektomie kam es jedoch nicht zum erwarteten Phosphatanstieg. Histologisch zeigte sich ein regressiv veränderter, knotig durchsetzter Schilddrüsenlappen ohne Anhalt für Malignität. Im Verlauf konnte trotz Substitution mit Decostriol und Phosphat kein ausreichender Anstieg des Phosphatspiegels erreicht werden. Zur weiteren Tumorsuche erfolgte die Durchführung eines DOTATATE PET-CT. Dieses zeigte eine hyperdense, subkutan gelegene Raumforderung im Bereich der Plantaraponeurose des rechten Fußes mit hochpathologischer Nuklidbindung. Der Tumor wurde exstirpiert und bereits einen Tag postoperativ war der Phosphatspiegel in den Normbereich angestiegen. Bei multiplen pathologischen Frakturen sollte an die seltene Ursache einer tumorinduzierten Osteomalazie gedacht und der Phosphatspiegel bestimmt werden. Zur Tumorlokalisation ist die Durchführung einer DOTATATE PET-CT zu empfehlen.

\section{PS17}

Assoziation von Single Nucleotid Polymorphismen im FGFR4- und Klotho-Gen mit linksventrikulärer Hypertrophie bei chronisch nierenkranken Patienten - eine Mendalian Randomisation Studie

Alexander B. Sellier; Sarah Seiler-Mußler; Insa E. Emrich;

Prof. Dr. med. Danilo Fliser; Adam M. Zawada; Gunnar H. Heine

Universitätsklinikum des Saarlandes

Zielstellung: Erhöhte Plasmaspiegel des Fibroblast Growth Factor 23 (FGF23) prädizieren zukünftige kardiovaskuläre Ereignisse bei chronisch nierenkranken Patienten. Rezente tierexperimentelle Studien suggerieren, dass erhöhte Plasmaspiegel von $\mathrm{FGF}_{23}$ via Aktivierung des FGF-Rezeptors 4 (FGFR4) eine linksventrikuläre Hypertrophie direkt induzieren. In konkurrierenden Studien erwies sich jedoch nicht ein Anstieg von FGF23, sondern eine Verminderung der Plasmaspiegel des löslichen FGF23 Korezeptors Klotho als Induktor der kardialen Hypertrophie. Um die klinische Relevanz der tierexperimentellen Daten zu überprüfen, untersuchten wir in der prospektiven CARE FOR HOMe Studie, inwieweit das Vorliegen genetischer Varianten von FGFR4 einerseits, von Klotho andererseits, mit linksventrikulärer Hypertrophie bei Studienbeginn und mit dem Auftreten inzidenter kardialer Dekompensationen assoziiert ist.

Methode: Die CARE FOR HOMe Studie rekrutierte 544 chronisch nierenkranke Patienten in GFR Kategorien G2 bis G4. Von 519 Teilnehmern wurde DNA isoliert und Single Nucleotid Polymorphismen für FGFR4 (Gly388Arg) und Klotho (Phe352Val) mittels qualitativer Real-Time PCR genotypisiert. Die Echokardiographien wurden zum Studieneinschluss durchgeführt $(\mathrm{N}=458)$ und folgten den Empfehlungen der American Society of Echocardiography. Über einen Zeitraum von 4,2 $\pm 2,1$ Jahren wurde bei den Teilnehmern das Auftreten des primären Endpunktes (kardiale Dekompensation) erfasst. 
Ergebnisse: Patienten mit unterschiedlichem FGFR4-Genotyp unterschieden sich nicht signifikant im Left Ventricular Mass Index (LVMI) (Gly/Gly: 91,7 $\pm 28,5 \mathrm{~g} / \mathrm{m}^{2}$, Gly/Arg: 91,4 $\pm 24,4 \mathrm{~g} / \mathrm{m}^{2}$, Arg/Arg: $93,9 \pm 28,7$ $\left.\mathrm{g} / \mathrm{m}^{2}, \mathrm{p}=0,861\right)$. Patienten mit unterschieden Klotho-Genotyp unterschieden sich zwar ebenso nicht signifikant bzgl. LVMI, jedoch hatten Patienten mit Genotyp Val/Val tendenziell höhere LVMI-Werte (Phe/ Phe: $91,1 \pm 25,4 \mathrm{~g} / \mathrm{m}^{2}$, Phe/Val: $92,8 \pm 28,2 \mathrm{~g} / \mathrm{m}^{2}$, Val $/$ Val: $100,9 \pm 48,0 \mathrm{~g} /$ $\mathrm{m}^{2}, \mathrm{p}=0,379$ ). Innerhalb des Nachbeobachtungszeitraums erlitten 104 Patienten eine kardiale Dekompensation. Weder FGFR4 noch Klotho Polymorphismen waren in univariaten Analysen mit Auftreten einer kardialen Dekompensation assoziiert (log rank test: FGFR4: $p=0,116$; Klotho: $p=0,853$ )

Schlussfolgerung: Genetische Varianten von FGFR4 und Klotho sind bei chronisch nierenkranken Patienten weder mit echokardiographischen Parametern einer linksventrikulären Hypertrophie noch mit dem Auftreten einer kardialen Dekompensation assoziiert, was weitere Studien an größeren Patientenpopulationen erforderlich macht, bevor rezente tierexperimentelle Ergebnisse in die klinische Nephrologie übersetzt werden können.

\section{PS18}

Strong age-dependent effects on migration of synovial fibroblasts obtained from patients with rheumatoid arthritis or osteoarthritis under dopamine receptor stimulation Lina van Nie'; Prof. Dr. Stefan Rehart ${ }^{2}$; PD Dr. Elena Neumann;; Prof. Dr. med. Ulf Müller-Ladner'; Dr. Silvia Capellino ${ }^{3}$

${ }^{1}$ Kerckhoff Klinik Bad Nauheim, Justus-Liebig-Universität Giessen; ${ }^{2}$ Markus Hospital, Frankfurt am Main; ${ }^{3}$ Leibniz Research Centre for Working Environment and Human Factors

Background: Preventing Synovial fibroblasts (SF) form migrating into the adjacent bone and cartilage is a very desirable target in rheumatoid arthritis (RA) therapy, as in doing so, both joint destruction and disability can be avoided. Our previous studies (Capellino $S$ et al, A\&R 2014) already showed a significant influence of dopamine on IL6 and IL8 release in RASF. Therefore, we suggest a possible impact of specific dopamine receptor (DR) activation on joint invasion and destruction in RA.

Materials and methods: SF were obtained from RA and osteoarthritis (OA) patients undergoing knee joint replacement surgery (mean age: $74.3 \pm 11.3 y r s$ at $O A$ and $73.7 \pm 10.3$ yrs at RA patients). For a better understanding of DR distribution within the synovium and especially in the invasion zone, immunohistochemistry was performed for all 5 DR subtypes. In addition, SF migration and motility assays and ELISAs for $M_{M} P_{3}$ and proMMP1 were performed under $D_{1}$-like (D1DR and $\left.\mathrm{D}_{5} \mathrm{DR}\right)$ and $\mathrm{D}_{2}$-like ( $\mathrm{D}_{2} \mathrm{DR}, \mathrm{D}_{3} \mathrm{DR}$ and $\left.\mathrm{D}_{4} \mathrm{DR}\right)$ receptor stimulation, each in different concentrations.

Results: The DR-subtypes D1DR, D2DR and D4DR are higher expressed on SF nearby the invasion zone between synovium and cartilage. It could be observed that migration of RSAF and OASF is highly correlated with the patients' age at surgery. While younger patients (? 75years) show an increase in migration up to $78 \%$, the older ones (? 75years) show a reduced migration up to $50 \%(p=0.0009 ; r=0.69,0 A$ $\mathrm{n}=8$; RA $\mathrm{n}=7$ ). Interestingly, no difference between D1-like and D2-like receptor stimulation could be observed. The same trend could be seen in the motility assay. (OA $n=6 ; R A n=6) . M M P_{3}$ and proMMP1 levels were also altered under $D R$ activation. $(O A n=3 ; R A n=3)$.

Conclusion: The high DR expression near the invasion zone suggests a direct role of dopamine on RASF and as a result on cartilage invasion. This was confirmed in the in vitro assays. Based on our results, further ongoing experiments will help us to understand the therapeutic potential of the dopamine pathway in RA. We are currently in- vestigating, if the observed age dependent changes in migration are due to altered DR expression or to DR sensitivity.

\section{PS19}

Veränderungen von NUCB2/Nesfatin-1 im Plasma stationärer Adipositas-Patienten nach therapeutischer

\section{Beeinflussung von Angst}

Dr. Tobias Hofmann'; Elena Weibert'; Dr. Anne Ahnis';

Dr. med. Alexander Obbarius ${ }^{2}$; Dr. med. Ulf Elbelt ${ }^{2}$;

Prof. Dr. med. Matthias Rose²; Prof. Dr. med. Burghard F. Klapp²;

PD Dr. Andreas Stengel ${ }^{2}$

${ }^{1}$ Charité Universitätsmedizin Berlin; ${ }^{2}$ Charité - Universitätsmedizin Berlin

Einleitung: Neben anorexigenen Eigenschaften bei der Regulation von Hunger und Sättigung zeigen sich zunehmend Hinweise auch für eine Beteiligung von NUCB2/Nesfatin-1 (NF-1) bei der Regulation von Angst und weiteren emotionalen Stresskorrelaten, welche offensichtlich geschlechtsspezifisch reguliert sind. Wir untersuchten den Zusammenhang von NF-1-Plasma-Spiegeln mit Angst bei adipösen Männern und Frauen sowie deren Veränderung während stationärer Behandlung. Erwartet wurden bei abnehmenden Angstwerten eine Verminderung der NF-1-Spiegel bei Frauen und eine Erhöhung bei Männern.

Methoden: Wir analysierten 69 aufgrund somatischer und psychischer Komorbidität bei Adipositas (nicht zwingend wegen einer Angststörung) stationär behandelte Patienten (44 weiblich, 25 männlich; BMl: $50.2 \pm 9.5$ kg/m2; 31.8 kg/m2-76.5 kg/m2; Alter: 45.0 \pm 12.4 Jahre). NF-1-Plasma-Spiegel wurden mit ELISA bestimmt. Angst (GAD-7) wurde zur gleichen Zeit psychometrisch bestimmt. Alle Messungen wurden sowohl zu Beginn der Behandlung durchgeführt als auch im Verlauf zu dem Zeitpunkt, an dem eine klinisch relevante Verbesserung der Angstwerte (?5 Punkte auf dem GAD-7) erreicht bzw. verfehlt wurde.

Ergebnisse: NF-1 korrelierte positiv mit den Angstwerten bei Frauen vor $(r=0.411 ; p=0.006)$ und nach $(r=0.301 ; p=0.047)$ der Behandlung. Bei Männern war eine negative Korrelation nach der Behandlung zu beobachten, die statistische Signifikanz vor der Behandlung wurde knapp verfehlt $(r=-0.381 ; p=0.059)$. Weder Frauen $(n=19 ;$ vor vs. nach Behandlung; $0.49 \pm 1.00 \mathrm{ng} / \mathrm{ml}$ vs. $0.38 \pm 0.72 \mathrm{ng} / \mathrm{ml} ; \mathrm{p}=0.687$ ) noch Männer ( $n=9 ; 0.17 \pm 0.31 \mathrm{ng} / \mathrm{ml}$ vs. $0.19 \pm 0.36 \mathrm{ng} / \mathrm{ml} ; \mathrm{p}=0.427$ ), die sich signifikant hinsichtlich ihrer Angstwerte verbessert hatten $(p<0.001)$, zeigten signifikante Veränderungen ihrer NF-1-Spiegel, auch wenn die Richtung der Veränderungen mit einer gegenläufigen Regulation bei Frauen und Männern wie erwartet ausfiel (Frauen:-23.3\%; Männer: $+12.4 \%)$.

Schlussfolgerungen: Der Zusammenhang zwischen NF-1 und Angst ist bei Frauen und Männern vor und nach Angstreduktion invers reguliert mit positiver Korrelation bei Frauen und negativer Korrelation bei Männern (allerdings keine Signifikanz bei Männern vor Behandlung). Hingegen veränderten sich die NF-1-Spiegel nicht wie erwartet signifikant mit der Verbesserung der Angst. Dies könnte an zu kleinen Stichproben insbesondere in den Subgruppen mit Angstreduktion und an insgesamt zu niedrigen Angstausgangswerten liegen, da die erwarteten Zusammenhänge als Trend durchaus erkennbar sind.

\section{PS20}

Hypokaliämische thyreotoxische periodische Paralyse als Erstmanifestation eines Morbus Basedows

Dorisandra Dumbrava; Dr. med. Hendrik Schmidt; Dr. med. Jörg Mittag

Einleitung: Die periodische hypokaliämische Paralyse ist eine seltene neurologische Krankheit mit familiär oder sporadisch auftretenden 
Formen im Zusammenhang mit Hyperthyreose, Hyperaldosteronismus und Barium-Intoxikation. Bei der Hyperthyreose führen die vermehrt zirkulierenden Schilddrüsenhormone zu einer Steigerung der Natrium-Kalium-ATP-ase mit vermehrtem Einstrom in die Zelle, konsekutiver Hypokaliämie und Muskelparese. Kasuistik: Ein 26-jähriger Patient asiatischer Abstammung stellte sich mit rezidivierenden, krampfartigen Schmerzen in den Unterschenkeln und den Oberschenkeln (v. a. morgens), gefolgt von Paraparese vor. Er berichtete weiterhin über Unruhe, vermehrtes Schwitzen und leichte subjektive Tachykardie. In der letzten Zeit bestanden eine Neigung zu Diarrhoen und ein Gewichtverlust(5kg). Keine Vorerkrankungen. Keine Vormedikation. Keine Traumata. Das toxikologische Screening war negativ.

Diagnostik: Der internistische Status zeigte bis zur Paraparese keine Auffälligkeiten. Der neurologische Status zeigte keinen meningealen Reiz-und keine Dehnungszeichen. Der Hirnnerven-Status war intakt. Es zeigte sich eine proximal betonte Schwäche der Beine. Die MER der unteren Extremitäten waren schwer auslösbar. EKG: Sinustachykardie (HF $110 \mathrm{bpm}$ ). Laborchemisch fiel eine manifeste Hyperthyreose auf (TSH $<0.005$ ?/L, FT3 19,71 pmol/L, $\mathrm{FT}_{4} 43,3 \mathrm{pmol} / \mathrm{L}$ ). Es fiel weiterhin eine ausgeprägte Hypokaliämie auf (Kalium 2,25 mmol/L). Zur weiteren Abklärung der manifesten Hyperthyreose wurde eine Sonographie der Schilddrüse veranlasst. Es zeigte sich eine Echoarmut des Schilddrüsengewebes bei deutlicher Inhomogenität und erhöhtem Volumen. Die Doppler-Sonographie zeigte eine vermehrte Durchblutung der gesamten Schilddrüse. Bei klinischem und sonographischem V.a. Morbus Basedow wurden die TSH-Rezeptor - Autoantikörper bestimmt. Diesen waren deutlich erhöht, so dass unsere Erstdiagnose Morbus Basedow sich bestätigt hat

Therapie: Der Patient wurde aufgrund der starken Hypokälimie und der oben geschilderten Beschwerdekonstellation auf unsere internistische Wachstation aufgenommen. Es wurde eine intravenöse Kaliumsubstitution unter Kreislaufs-Monitoring begonnen. Weiterhin wurde eine Thyreostatika Therapie i. v. eingeleitet. Im Rahmen der Tachykardie erhielt der Patient kurzfristig Betablocker p. o. (Propranolol) Unter Kaliumzufuhr und Elektrolytenausgleich bildete sich die Paraparese innerhalb von ein paar Stunden zurück.

Schlussfolgerung: Beim Auftreten von periodischen Lähmungen bei jungen Patienten sollte trotz der Seltenheit dieser Krankheit in der westlichen Bevölkerung an eine periodische hypokaliämische Parese bei Thyreotoxikose gedacht werden. Die Lähmung kann sich symmetrisch oder asymmetrisch präsentieren. Die Hirnnerven sind typischerweise ausgenommen. Das Sensorium ist nicht beeinträchtigt und die Atemmuskulatur bleibt verschont. Die Korrektur der hyperthyreoten Stoffwechsellage ist eine Prophylaxe für das Auftreten neuer neurologischer Episoden.

\section{PS21}

\section{Acetyliertes ADMA und acetyliertes SDMA als kardiovaskuläre Prognosefaktoren bei chronisch nierenkranken Patienten?}

Dr. med. Insa Emrich; Dr. Adam M. Zawada; Dr. Jens Martens-Lobenhoffer; Prof. Dr. med. Danilo Fliser; Prof. Dr. med. Gunnar H. Heine;

Prof. Dr. Stefanie M. Bode-Böger

Chronisch nierenkranke Menschen haben eine erhöhte kardiovaskuläre Morbidität und Mortalität, die sich nicht allein durch traditionelle kardiovaskuläre Risikofaktoren erklären lassen. Vielmehr tragen zum kardiovaskulären Risiko nicht-traditionelle kardiovaskuläre Risikofaktoren bei. Asymmetrisches und symmetrisches Dimethylarginin (ADMA; SDMA) werden seit Jahren als solche nicht-traditionellen Risikofaktoren betrachtet. Kürzlich konnte die Acetylierung von ADMA und SDMA als ein neuer Abbaumechanismus beider Metabolite im Mausmodell evaluiert werden, wobei diese acetylierten Formen stärker mit der Nierenfunktion assoziiert waren als die ursprünglichen
Metabolite. Wir überprüften daher bei chronisch nierenkranken Menschen, ob acetyliertes ADMA und acetyliertes SDMA stärker bei abnehmender Nierenfunktion akkumulieren als ADMA und SDMA, und ob beide als neue kardiovaskuläre Prognosemarker für chronisch nierenkranke Patienten zu betrachten sind. Wir analysierten 528 CKD Patienten der KDIGO Stadien $\mathrm{G}_{2}$ bis $\mathrm{G}_{4}$, die seit 2008 in die fortlaufende CARE FOR HOMe Studie eingeschlossen wurden. ADMA, SDMA, acetyliertes ADMA und acetyliertes SDMA wurde aus bei Einschluss asservierten Plasmaproben bestimmt und die Patienten jährlich bezüglich des Auftretens von kardiovaskulären Ereignissen nachverfolgt. Insgesamt wurden die Patienten über $4.6 \pm 2.0$ Jahre nachbeobachtet. Das mittlere Plasma-ADMA betrug 0.49 [0.44; 0.55] $\mu \mathrm{mol} / \mathrm{l}$, das Plasma-SDMA 0.72 [0.59; 0.98] $\mu \mathrm{mol} / \mathrm{l}$, das acetylierte Plasma-ADMA (Ac-ADMA) $1.24[0.74 ; 2.16] \mathrm{nmol} / \mathrm{I}$ und das acetylierte Plasma-SDMA (Ac-SDMA) $8.42[3.60 ; 19.12] \mathrm{nmol} / \mathrm{l}$. Es zeigte sich zunächst wie vermutet eine stärkere Assoziation zwischen den acetylierten Metaboliten und der glomerulären Filtrationsrate im Vergleich zu ADMA und SDMA und der glomerulären Filtrationsrate. 144 Patienten erlitten im Beobachtungszeitraum ein kardiovaskuläres Ereignis. Im univariaten Modell zeigte sich eine signifikante Assoziation zwischen allen vier Metaboliten und dem Auftreten von kardiovaskulären Ereignissen, diese Assoziation bleibt allerdings im multivariaten Modell nach Korrektur für Nierenfunktion und traditionelle kardiovaskuläre Risikofaktoren nur für SDMA bestehen. Zusammenfassend sind die acetylierten Metabolite von ADMA und SDMA stärker mit der Nierenfunktion assoziiert als ADMA und SDMA selbst. Da allerdings alleinig SDMA im multivariaten Modell mit dem Auftreten von kardiovaskulären Risikofaktoren assoziiert ist, erscheint eine Bestimmung der acetylierten Formen von ADMA und SDMA im klinischen Alltag aktuell nicht zielführend.

\section{Innere Medizin im höheren Lebensalter}

\section{PS22}

\section{Calciphylaxie einer Nicht-Dialyse-Patientin bei}

Aortenklappenersatz - ein Antikoagulationsdilemma

Dr. med. Melanie Kandulski'; Dr. med. Arno Rütten²;

Prof. Dr. med. Peter Rene Mertens ${ }^{3}$; Dr. med. Christos Chatzikyrkou ${ }^{3}$

${ }^{1}$ Universitätsklinikum Magedeburg; ${ }^{2}$ Dermatopathologie Friedrichshafen;

${ }^{3}$ Universitätsklinikum Magdeburg

Eine leicht adipöse 60-jährige Patientin präsentierte sich mit schmerzhaften, zunächst livide verfärbten Indurationen im Bereich der Oberschenkel, die im Verlauf nekrotisch imponierten. Histologisch ergab sich das Bild einer Calciphylaxie. Die Patientin nahm aufgrund einer rheumatoiden Arthritis wöchentlich 7,5 mg Methotrexat und 2,5 mg Prednisolon pro Tag ein. Aufgrund eines mechanischen Aortenklappenersatzes erfolgte eine Antikoagulation mittels Phenprocoumon. Es war lediglich eine Nephropathie im Stadium 2 nach KDIGO bekannt. Das Kalziumphosphatprodukt zeigte sich ausgeglichen und das Parathormon befand sich im Normbereich. Es erfolgte eine Beendigung der Dauertherapie mit Kalzium und Vitamin D. Aufgrund des bekannten erhöhten Risikos der Entwicklung einer Calciphylaxie unter der Therapie mit Phenprocoumon wurde diese auf eine off-label-Therapie mittels $5 \mathrm{mg}$ Apixaban zweimal täglich umgestellt. Die immunsuppressive Therapie mit Methotrexat und Prednisolon wurde aufgrund fehlender Alternativen bei multiplen Unverträglichkeiten in der Vergangenheit unverändert fortgeführt. Nach konservativer Ablösung der Nekrosen durch die Verwendung von Hydrogelen erfolgten nach fünf Monaten eine chirurgische Nekrosektomie, eine vorübergehende VAC-Therapie sowie die anschließende plastische Deckung der Defekte. Nach 6 Monaten präsentierten sich die ehemaligen Calciphylaxieherde vollständig abgeheilt. Neue Herde sind nach Umstellung der Therapie nicht aufgetreten. Das Krankheitsbild der 
Calciphylaxie ist sehr selten und tritt fast ausschließlich bei Dialysepatienten oder bei nierentransplantierten Patienten auf.

\section{PS23 \\ Gastrointestinal bleeding diagnosed by capsule endoscopy - a 13 years' single center experience \\ Dr. med. Alexander Hann; Thomas Seufferlein; Eugen Zizer \\ Universitätsklinikum Ulm}

Background: Capsule endoscopy (CE) is a well-established technique for the diagnosis of gastrointestinal bleeding located in the small bowel. The introduction of new oral anticoagulants increased the risk of gastrointestinal bleeding, especially in the elderly patients. Additionally new developments like the enhanced battery capacity and improved image quality might have raised its popularity during recent years. The purpose of this study is to evaluate the total number of $\mathrm{CE}$ studies and the percentage of findings over time.

Methods: All CE examinations performed for the detection of a small bowel gastrointestinal bleeding during the years 2004 to September 2016 were analyzed. Additionally baseline characteristics of the patients and findings using CE were evaluated.

Results: We identified a total of 553 CE. The mean number of examinations per year rose from 32 in the years $2004-2012$ to 65 in the years 2013 to 2016. Additionally the percentage of positive findings including overt bleeding and identification of a potential bleeding source rose from mean $59 \%$ to $74 \%$ respectively.

Conclusion: The total number of $\mathrm{CE}$ and positive findings presented an increase during the last four years. Possible reasons like the introduction of new anticoagulants have to be evaluated in a further examination.

\section{PS24}

Effekt eines physiologischen Stressmodells auf den Calcium-Phosphat Haushalt und Fibroblast

Growth Factor 23 bei nierengesunden Probanden

Marc-Sebastian Baier'; Lucie Bauer²; Dr. Insa E. Emrich²;

Adam Zawada' ${ }^{2}$ Prof. Dr. med. Danilo Fliser ${ }^{2}$; Prof. Dr. Jürgen Scharhag ${ }^{3}$;

Prof. Dr. Gunnar Heine ${ }^{2}$

${ }^{1}$ Innere Medizin IV; ${ }^{2}$ Klinik für Innere Medizin IV; ${ }^{3}$ Institut für Sport- und

Präventivmedizin

Ein hoher Plasmaspiegel des phosphaturischen Hormones Fibroblast Growth Factor 23 (FGF-23) ist bei nierenkranken und nierengesunden Menschen mit kardiovaskulären Erkrankungen assoziiert, wobei die Kausalität bislang unklar ist. Tierexperimentelle Daten weisen auf eine erhöhte Expression von FGF-23 bei erhöhter Sympathikusaktivität hin und suggerieren somit, dass eine chronische Herzinsuffizienz mit konsekutiver Sympathikusaktivierung (SA) Ursache und nicht Folge erhöhter FGF-23 Plasmaspiegel sein kann. Da die klinische Bedeutung dieser Verknüpfung noch ungeklärt ist, soll der Einfluss einer SA im physiologischen Stressmodell einer akuten körperlichen Aktivität auf den Calcium-Phosphat Haushalt mit Fokus auf FGF-23 untersucht werden. In der Fit@Home Studie wurden 15 männliche gesunde Probanden im Zeitraum von März bis November 2015 eingeschlossen. Bei der ausführlichen Eingangsuntersuchung erfolgte eine standardisierte Fahrradergometrie (FE) zur Ermittlung der individuellen anaeroben Schwelle (IAS) und der Zielherzfrequenzen. In randomisierter Reihenfolge absolvierten die Probanden im Abstand von einer Woche eine intensive FE bei $90 \%$ der IAS und eine hochintensive FE bei $110 \%$ der IAS. Messungen von c-terminalem (Immuntopics; San Clemente; CA) und intaktem (Immuntopics; San Clemente; CA) FGF-23 und von weiteren Parametern des Calcium-Phosphat-Haushaltes erfolgten viermalig (vor der FE und $5 \mathrm{~min}, 90 \mathrm{~min}$ sowie $24 \mathrm{~h}$ danach). Die Probanden waren im Mittel $25 \pm 3$ Jahre alt, 1,79 $\pm 0,07 \mathrm{~m}$ groß, $79 \pm 13$ $\mathrm{kg}$ schwer und hatten eine mittlere eGFR von $110 \pm 17 \mathrm{ml} / \mathrm{min} / 1,73 \mathrm{~m}^{2}$. Da zwei Probanden nicht die Vorgabe der intensiven FE (Belastungszeitraum: $60 \mathrm{~min}$ ) erreichten, wurden sie bei den anschließenden Analysen dieser Belastung nicht berücksichtigt. Weder die intensive noch die hochintensive Fahrradergometrie induzierten einen signifikanten Anstieg von c-terminalem (intensiv: $p=0,763$; hochintensiv: $p=0,184$ ) und intaktem FGF-23 (intensiv $p=0,582$; hochintensiv $p=$ $0,135)$. Jedoch stieg Plasma Phosphat trotz Erhöhung der fraktionellen Phosphatausscheidung, welche in der intensiven, nicht jedoch in der hochintensiven Belastung Signifikanzniveau erreichte. Das Plasma Calcium stieg bei hochintensiver, nicht jedoch bei intensiver Belastung mit Abnahme der fraktionellen Calciumausscheidung. Die Daten unserer Studie untermauern nicht die in experimentellen Studien postulierte Hypothese, dass eine SA einen signifikanten FGF-23 Anstieg induziert. Als Limitation muss bedacht werden, dass wir gesunde Probanden und keine Patienten mit Herzinsuffizienz untersuchten. Im nächsten Schritt ist zu prüfen, ob bei dieser Patientengruppe FGF23 unter körperlicher Belastung ansteigt. Die signifikanten Anstiege von Plasma Phosphat und Calcium suggerieren eine belastungsinduzierte Aktivierung FGF-23 unabhängiger Mediatoren.

\section{PS25}

\section{Prädiktoren von 30 Tage-Mortalität bei Clostridium}

\section{difficile-assoziierter Enterocolitis}

Katrin Claudia Katzer'; Dr. Stefan Hagel; Dr. Tony Bruns;

Prof. Dr. Andreas Stallmach

${ }^{1}$ Universitätsklinikum Jena

Hintergrund: Infektionen durch Clostridium difficile (CDI) zeigen eine steigende Inzidenz und sind mit verlängertem Krankenhausaufenthalt sowie erhöhter Mortalität verbunden. Ziel dieser Arbeit ist es, unabhängige Prädiktoren für CDI-bedingte Mortalität zu identifizieren, um ein einheitliches Vorhersagemodell in Bezug auf die schwer verlaufende Infektionen zu erstellen.

Methoden: Es wurde eine retrospektive Analyse von 127 Patienten mit CDI, die im Zeitraum 09/2011 bis 12/2012 stationär in unserem Zentrum behandelt wurden, durchgeführt. Die Identifikation von Prädiktoren der 30-Tage Martalität erfolgte mittels ROC-Analyse und logistischer Regression. Ergebnisse: Die 30-Tage Mortalität nach Diagnose einer CDI betrug 35/127 (28\%). Parameter, die bei Diagnosestellung signifikant mit Mortalität assoziiert waren, waren erhöhtes C-reaktives Protein (optimaler cut-off: ?121 mg/l; univariate odds ratio 3,80; $95 \% \mathrm{Cl}$ $1,64-7,80 ; p=0,003$ ) und ein erniedrigter systolischer Blutdruck (optimaler cut-off: ?104 mmHg; univariate odds ratio 3,73; $95 \% \mathrm{Cl}$ 1,63-8,53 ; $p=0,002$ ). Eine innerhalb von 6 Tagen auftretende Nierenfunktionsstörung (Kreatininanstieg $>1,5$-fach im Vergleich zum Diagnosetag; univariate odds ratio 5,$61 ; 95 \% \mathrm{Cl} 1,94-16,26 ; p=0,035)$ war ebenfalls prädiktiv für ein Versterben innerhalb von 30 Tagen. Die drei identifizierten Prädiktoren waren unabhängig voneinander in der multivariaten logistischen Regression mit Mortalität assoziiert und erlaubten eine korrekte Prädiktion in dieser Kohorte von $73 \%$ am Diagnosetag und $76 \%$ an Tag 6. Die Kombination der identifizierten Parameter waren den verschiedenen publizierten Definitionen einer schweren CDI (Definitionen nach Zar, American College of Gastroenterology (ACG), European Society of Clinical Microbiology and Infectious Diseases (ESCMID), Society for Healthcare Epidemiology of America(SHEA)) überlegen.

Schlussfolgerungen: Hohes CRP und erniedrigter systolischer Blutdruck bei Diagnose sowie Auftreten einer Nierenfunktionsstörung im Verlauf sind geeignet, schwere Fälle von CDI mit hoher Mortalität zu identifizieren. 


\section{PS26}

Diverging prevalence of chronic kidney disease by various formula, awareness and associated risk factors in the elderly. Results from the Berlin Aging Study II (BASE-II).

Dr. Maximilian König; Prof. Dr. Maik Gollasch; PD Dr. Ilja Demuth;

Prof. Dr. med. Elisabeth Steinhagen-Thiessen

Charité - Universitätsmedizin Berlin

Background: In aging populations with an ever-growing burden of risk factors like obesity, diabetes and hypertension, chronic kidney disease (CKD) is on the rise. Approximately $20 \%$ in the age-group of $60-$ 69 years and at least $35 \%$ aged 70 years and older were shown to have impaired kidney function. In particular in the elderly, there is controversy as to which formula for the estimation of GFR should be used, especially as recently novel equations for estimating the glomerular filtration rate (eGFR) have emerged, which have not been applied comprehensively to older adults. Moreover, often albuminuria is not provided in the definition of CKD, leading to only vague prevalence estimates. Furthermore, data on CKD awareness in the elderly are sparse.

Objective: To determine the prevalence of CKD among older adults by eGFR and albuminuria, compare the performance of 6 established and novel eGFR formulas, explore risk factors and assess the awareness of CKD in a large cohort of community-dwelling elderly from Germany.

Methods: 1628 subjects from the Berlin Aging Study II (BASE-II) were included in this analysis (mean age 68.7 years; $51.2 \%$ female). Extensive cross-sectional data with respect to socio-demographics, lifestyle, medication and diagnoses were inquired during structured interviews and medical examination. Estimated GFR was calculated using the FAS (Full Age Spectrum), the Lund-Malmö revised (LMr), the MDRD, the BIS1, the Cockcroft-Gault and the CKD-EPI equations. Urinary albumin excretion was expressed as albumin/creatinine ratio (ACR) in $\mathrm{mg} / \mathrm{g}$. GFR $<60 \mathrm{ml} / \mathrm{min} / 1.73 \mathrm{~m} 2$ and/or $A C R>30 \mathrm{mg} / \mathrm{g}$ were considered as criteria for CKD.

Results: $77.1 \%$ of subjects had hypertension, $12.4 \%$ had diabetes and $18.3 \%$ were obese. Prevalence of CKD strongly depended on the eGFR equations used: $25.4 \%$ (FAS), $24.6 \%$ (BIS1), 23.1\% (Lund-Malmö revised), $19.3 \%$ (Cockcroft-Gault), $16.4 \%$ (CKD-EPI) and $14.7 \%$ (MDRD), respectively. A relevant proportion of all subjects (7.2\%) had isolated albuminuria (ACR ?30 mg/g). Notably, among subjects with CKD, only $3.9 \%$ were aware of their condition. Polypharmacy (regular use of 5 or more drugs), age, BMI, coronary artery disease, non-HDL-cholesterol and female sex were identified to be independently associated with CKD in multiple regression analysis. After full adjustment, polypharmacy was associated with a $54 \%$ increased risk of CKD.

Conclusions: CKD is common among older adults in Germany, but awareness is low. The FAS equation detects higher rates of CKD than MDRD and CKD-EPI, which are most widely used at present. Also, when CKD is defined based on eGFR and albuminuria, considerably more people are identified than by eGFR alone. Finally, polypharmacy is an underrated risk factor for CKD in the elderly.

\section{PS27}

p53 Regulation mit Destabilisierung des Darmepithels - Folgen der bakteriellen Infektion bei spontan bakterieller Peritonitis? Marika Haderer; Claudia Kunst; Elisabeth Aschenbrenner; Kirstin Pollinger; Johanna Middendorf; Sophie Schlosser; Martina Müller-Schilling

Einleitung: Spontan bakterielle Peritonitis (SBP) ist eine lebensgefährliche Komplikation bei fortschreitender Leberzirrhose. Es handelt sich um eine Infektion des Bauchwassers (Aszites) in Abwesenheit ei- nes intra-abdominellen, chirurgisch entfernbaren Entzündungsherds. Maßgebend für eine SBP ist eine bakterielle Translokation, bei der Darmbakterien oder deren Bestandteile aus dem Darm in das splanchische Abstromgebiet und das Lymphsystem wandern. Im Zusammenhang mit diesem leaky gut Phänomen wird der Einfluss von Zellkontakt vermittelnden Proteinen diskutiert. Häufigste Erreger der SBP sind Escherichia coli (E. coli), Enterokokken, Streptokokken und Klebsiellen. Mit Beginn der bakteriellen Translokation ist eine antibiotische Behandlung des Patienten erforderlich. Um eine SBP frühzeitig diagnostizieren und eine Behandlung gezielt und rechtzeitig einleiten zu können, ist ein Verständnis der Pathomechanismen der SBP essentiell.

Ziel: Im Hinblick auf die Entwicklung von Früherkennungsystemen, sollen die der bakteriellen Translokation zugrundeliegenden Mechanismen bei SBP identifiziert werden.

Methodik: Die humanen Darmepithelzelllinien Caco-2 (p53-defizient) und HCT-116 (p53 wildtyp) wurden in vitro mit E.coli in verschiedenen MOI (MOI 0, 1, 5 und 10) für 2-4h koinkubiert. Dieser Versuchsansatz diente der Studie von Einflüssen SBP-relevanter Bakterien auf die Zell-Zell-Kontakte des Darmepithels. Effekte auf RNA und Proteinebene von Occludin, E-Cadherin (Zell-Zell-Kontakte) und der P53-Familie wurden mittels qPCR und Western Blot analysiert. Ein IL-8 ELISA wurde zur Untersuchung des Immunstatus der HCT-116 Zellen herangezogen.

Ergebnis: Die Koinkubation mit E. coli führte bei den HCT-116 Zellen in Abhängigkeit der $\mathrm{MOI}$ zu einer starken Reduktion des tight junction Proteins Occludin sowie des adherens junction Proteins E-Cadherin. Zudem war die Proteinmenge von p53 und p73 MOl-abhängig verringert. Caco-2 Epithelzellen wiesen nach E. coli Koinkubation ebenfalls leicht reduzierte Mengen an Occludin und E-Cadherin auf. Auf RNA-Ebene wurden führte die bakterielle Stimulation zu geringfügigen Anstiegen der Zell-Zell-Kontakte. Bei den HCT-116 Zellen stieg die IL-8- Expression nach bakterieller Stimulation an.

Schlussfolgerung: Bakterien haben destabilisierende Effekte auf die Integrität der epithelialen Barriere und besitzen darüber hinaus einen regulatorischen Einfluss auf die p53-Familie von Transkriptionsfaktoren. Unsere Hypothese ist daher, dass ein bakterieller Schutzmechanismus existiert, welcher mittels Hemmung der p53 Familie die bakterielle Replikation fördert.

\section{PS28}

Prognostic Impact of Anemia Etiology in a Contemporary Cohort of Patients Undergoing Transcatheter Aortic Valve Implantation Dr. Tobias Andreas Rheude'; Costanza Pellegrini; Dr. Teresa Trenkwalder; Jonathan Michel; Dr. Nicola Patrick Mayr; PD Dr. Markus Kasel; Prof. Dr. Heribert Schunkert; Prof. Dr. Adnan Kastrati; Prof. Dr. Christian Hengstenberg; PD Dr. Oliver Husser ${ }^{1}$ Deutsches Herzzentrum München

Introduction: Anemia is a frequent co-morbidity in patients undergoing transcatheter aortic valve implantation (TAVI) with an adverse clinical impact. The prevalence and impact of different etiologies of anemia has not been investigated.

Methods and Results: 268 patients underwent TAVI and were included in the study (age of $81 \pm 6$ years and a logistic EuroScore of $16 \pm 12 \%$ ). Baseline anemia was defined as a hemoglobin level $<13 \mathrm{~g} / \mathrm{dL}$ in men and $<12 \mathrm{~g} / \mathrm{dL}$ in women. The etiology of anemia was classified as iron deficiency, renal or other causes. The primary endpoint was a composite of death or re-hospitalization for worsening heart failure during the first year after TAVI. Anemia was present in $47 \%$ (127/268) of the patients. The etiology of anemia was iron-deficiency in $41 \%(52 / 127)$, renal in $31 \%(40 / 127)$ and other causes in $28 \%$ (35/127). Patients with 
anemia had a significantly higher event rate compared to patients without ( $21 \%$ (27/127) vs. $8 \%$ (11/141); $p=0.002)$. Overall, all types of anemia had comparable outcomes. Specifically, the primary endpoint occurred in $23 \%$ of patients with iron-deficiency anemia (12/52), $21 \%$ with renal anemia (8/40), and $20 \%$ with other causes of anemia ( $7 / 35)$. In a multivariable Cox regression analysis baseline anemia was an independent predictor of the primary endpoint (hazard ratio $2.5,95 \%$ confidence interval [1.18-5.29]; $p=0.02$ ).

Conclusion: Anemia is common in contemporary TAVI-patients and independently predicts adverse outcome regardless of etiology. Whether prior treatment of potential reversible causes of anemia, especially iron deficiency, impacts prognosis remains to be investigated.

\section{PS29 \\ p73 - ein starker Aktivator der IGFBP4 Genexpression beim hepatozellulären Karzinom \\ Sebastian Heckel'; Dana Gschwind; PD Dr. Claudia Kunst; Elisabeth Aschenbrenner; Kirstin Pollinger; Sophie Schlosser; Prof. Dr. Martina Müller-Schilling \\ ${ }^{1}$ Universitätsklinkum Regensburg}

Hintergrund: Die $p_{53}$ Familienmitglieder $p_{53}, p_{63}$ und $p_{73}$ sind an der Regulation von Zellzyklus, Seneszenz und Apoptose beteiligt. In ihrer Funktion als Transkriptionsfaktoren und abhängig von ihrer jeweiligen Splicevariante - mit Transaktivierungsdomäne (TA) oder dominant negative (DN) - aktivieren oder inhibieren sie die Transkription spezifischer Zielgene. In Vorarbeiten wurde das Gen für Insulin-like growth factor binding protein 4 (IGFBP4) als potentielles Zielgen der p53-Familie mit prognostischer Relevanz im hepatozellulären Karzinom (HCC) identifiziert. Im Gegensatz zu p53 ist das IGF-System an der Regulation von Wachstum und Zellüberleben beteiligt. IGFBP4 wirkt hierbei als Inhibitor, der proliferative IGF-Effekte limitiert und in dieser Funktion möglicherweise mit p53-Proteinen interagiert. Ziel dieser Arbeit war daher die Charakterisierung des regulatorischen Einflusses der p53-Familie auf IGFBP4.

Methoden: Hep3B-Zellen wurden mit rAd-p53, -TAp63, -TAp73, -DNp63 und -DNp73 transfiziert. Die transkriptionelle Regulation von IGFBP4 wurde mittels real time qPCR bestimmt. Intra- und extrazelluläre IGFBP4 Proteinmengen wurden mittels Western Blot und ELISA ermittelt. Zur Identifikation potentieller Bindungstellen für Proteine der p53-Familie im IGFBP4 Lokus wurde eine Transfac Datenbankanalyse durchgeführt. Die so identifizierten Sequenzen wurden kloniert, deletiert und in Luciferase-Reporterassays analysiert, um die Bindung von p53-Proteinen zu evaluieren.

Ergebnisse: Die IGFBP4 Expression war in TAp73-transfizierten Hep3B-Zellen über 30-fach, in DNp63-transfizierten Zellen über 15fach und in p53-transfizierten Zellen 3 -fach erhöht. Eine Induktion von intrazellulärem IGFBP4-Protein wurde in allen transfizierten Zellen beobachtet, während extrazelluläres IGFBP nur nach Transfektion mit TAp73 und DNp63 nachweisbar war. Mittels Datenbankanalyse wurden im Intron 1 des IGFBP4-Gens zwei putative p73-Bindungsstellen identifiziert. Die Intron1-abhängige Luciferaseaktivität war in TAp73-transfizierten Zellen mehr als 20-fach erhöht. Diese Induktion war nach Deletion einer der beiden putativen Bindungsstellen um über $70 \%$ reduziert.

Schlussfolgerung: Diese Ergebnisse identifizieren das Gen des IGF Inhibitors IGFBP4 als neues p73-Zielgen beim HCC. Erstmals zeigen wir die Wechselwirkung von TAp73 und IGFBP4 und damit eine bisher unbekannte Assoziation des Netzwerks der p53 Familie und der IGF Signaltransduktion. Da wir in Vorarbeiten bereits IGFBP2 als weiteres Zielgen der p53-Familie identifizierten, unterstreichen diese Ergeb- nisse die Verbindung zwischen tumorinhibierenden Mechanismen durch die p53 Familie und IFG-abhängiger Zellproliferation. Unsere Hypothese ist daher, dass das spezifische Verhältnis dieser beiden Signalwege für Zellwachstum, Kanzerogenese und Therapieansprechen von entscheidender Bedeutung ist.

\section{PS30}

IGFBP2 - ein neues Zielgen der p53-Familie beim hepatozellulären Karzinom

Dana Gschwind'; Maximilian Lohse'; PD Dr. Claudia Kunst'; Elisabeth Aschenbrenner'; Kirstin Pollinger'; Sophie Schlosser; Prof. Dr. med. Martina Müller-Schilling ${ }^{1}$

${ }^{1}$ Universitätsklinikum Regensburg

Hintergrund: In einer Vielzahl von Tumoren, unter anderem dem hepatozellulären Karzinom (HCC), haben die Mitglieder der p53-Familie kanzerogene oder tumorsuppressive Eigenschaften. Die Transkriptionsfaktoren der p53-Familie ( $553, p 63, p 73)$ reagieren auf zellulären Stress mit der Regulation spezifischer Zielgene. Abhängig von der jeweiligen p 53 Splicevariante - mit Transaktivierungsdomäne (TA) oder dominant negativ (DN) - und den Eigenschaften der spezifischen Bindungsstelle (BS) werden diese Zielgene aktiviert oder unterdrückt. In Vorarbeiten identifizierten wir IGFBP2 (Insulin-Like Growth Factor Binding Protein 2) als potentielles Zielgen der p53-Familie mit prognostischer Relevanz beim HCC. Ziel dieser Arbeit war nun darzustellen, in welcher Weise die Regulation von IGFBP2 durch p53-Proteine erfolgt.

Methoden: $\mathrm{Hep}_{3} B$ Zellen wurden mit rAd-p53 und -TAp73 transfiziert. Die transkriptionelle Regulation von IGFBP2 wurde mittels qPCR bestimmt. Intra- und extrazelluläre IGFBP2-Proteinmengen wurden mit Western Blots und ELISA ermittelt. Eine Transfac Datenbankanalyse diente zur Identifizierung potentieller p53 und p73 BS im IGFBP2-Gen. Diese Sequenzen wurden kloniert, mutiert und in Luciferase-Reporterassays auf die Bindung von $\mathrm{p} 53$-Proteinen untersucht.

Ergebnisse: Die Transfektion mit TAp73 führte zu einer über 25-fach erhöhten IGFBP2-Expression und zu deutlich erhöhten intra- und extrazellulären IGFBP2-Proteinmengen, während in den Kontrollen kein IGFBP2-Protein nachweisbar war. Nach Transfektion mit p53 war die IGFBP2-Expression um bis zu 7-fach erhöht. Zwei potentielle p53 und p73 BS liegen in der Promotorregion, 5 weitere potentielle p73-BS und eine p53-BS wurden im Intron 1 des IGFBP2-Gens identifiziert. Die Intron 1-abhängige Luciferaseaktivität war in TAp73-transfizierten Zellen um bis zu 150-fach, in TAp53-transfizierten Zellen um bis zu 20-fach erhöht. Mutation und Deletion der identifizierten p53-BS in Intron 1 resultierten in einer bis zu 90\%igen Reduktion der Luciferaseaktivität in p53- und TAp73-transfizierten Zellen. Die Deletion einer der potentiellen p73-BS hatte eine um $85 \%$ verringerte Luciferaseaktivität in TAp73-transfizierten Zellen zur Folge.

Schlussfolgerung: Diese Ergebnisse identifizieren IGFBP2 eindeutig als neues Zielgen für TAp73 und p53 beim HCC. Mit dem Nachweis der Wechselwirkung von TAp73 und IGFBP 2 zeigen wir eine wichtige, bisher unbekannte Verbindung zwischen dem Netzwerk der p53-Familie und der IGF-Achse. Während p53-Proteine überwiegend tumor-inhibierende Effekte auf die Zelle haben, ist die IGF-Achse für proliferative Prozesse von entscheidender Bedeutung. Das Verhältnis dieser beiden wichtigen Signalwege scheint daher Tumoreigenschaften genau wie für Therapieansprechen maßgeblich zu beeinflussen. Unsere Ergebnisse erweitern somit nicht nur das Verständnis für kanzerogene Prozesse beim HCC, sondern indizieren auch, dass sich durch Feinabstimmung dieser Signalwege neue therapeutische Möglichkeiten beim HCC bieten. 


\section{Innere Medizin im höheren Lebensalter 2}

\section{PS31}

Notwendigkeit einer beidseitigen sonographischen Diagnostik bei Verdacht auf tiefe Beinvenenthrombose

Franziska Thum; Prof. Dr. med. Bettina- Maria Taute

Hintergrund und Ziel: Bei Verdacht auf tiefe Beinvenenthrombose (TVT) besteht Unklarheit über die Notwendigkeit einer beidseitigen sonographischen Diagnostik insbesondere dann, wenn die abzuklärende Symptomatik nur einseitig vorliegt. Das Übersehen einer kontralateralen TVT kann sowohl Ursache eines postthrombotischen Syndroms sein als auch das Risiko für Morbidität und Mortalität durch Progredienz und Lungenembolie (LAE) erhöhen. Ziel war es zu prüfen, wie hoch die Rate einer beidseitigen TVT in einem definierten TVT-Krankengut liegt und welche Patienten von einer beidseitigen Diagnostik profitieren.

Patienten und Methodik: In eine retrospektive monozentrische Studie wurden konsekutive Patienten (Pat.) mit TVT eingeschlossen, die in der angiologischen Funktionsabteilung des Universitätsklinikums Halle vorgestellt wurden und eine komplette sonografische Diagnostik beider Beine erhalten hatten. Neben der Rate bilateraler TVT interessierten prädisponierende Faktoren für eine bilaterale TVT.

Ergebnisse: Eingeschlossen wurden 936 Pat. (Männer 48,6\%, mittl. Alter $67+/-16,8$ J.) mit TVT. Eine bilaterale TVT bestand bei 323 Pat. (33,5\%). Häufige TVT-Ursachen waren: 32,4\% Tumor-assoziierte, 29,8\% postoperative und $25,4 \%$ im Rahmen einer Immobilisierung erworbene TVT neben $10,9 \%$ idiopathische TVT. Unter 640 Pat. mit unilateraler TVT wurde bei 59,2\% eine proximale, bei $26,6 \%$ eine distale TVT und bei $14,2 \%$ der Pat. eine isolierte Muskelvenenthrombose (MVT) beschrieben. Von 323 Pat. mit bilateraler TVT wiesen 7,4\% beidseitig eine proximale, $55,1 \%$ eine proximale und kontralateral eine distale und $31,9 \%$ beidseitig eine distale TVT auf. Bei 5,6\% bestanden beidseitig isolierte MVT. Eine bilaterale TVT fand sich signifikant häufiger bei koinzidenter LAE ( $p<0.0001)$, bei im Krankenhaus erworbener TVT $(p<0.0001)$, bei Pat. nach neurochirurgisch-intrakranieller Operation $(p<0.0001)$, bei Pat. mit Immobilisation $(p=0.03)$ und bei Pat. über 67 Jahren $(p=0.037)$. Bei $74,9 \%$ der Pat. $(n=721)$ lagen TVT-Symptome vor. Bei $25,1 \%$ der Pat. $(n=242)$ bestanden keine klinischen TVT-Symptome, davon hatten $36 \%(n=87)$ eine bilaterale TVT. 606 Patienten bestand eine einseitige TVT-Symptomatik. Bei 20,3\% dieser Pat. ( $n=123)$ wurde eine bilaterale TVT diagnostiziert. Die multivariate Analyse zeigte in dieser Subpopulation signifikant häufiger eine bilaterale TVT bei Vorliegen einer distalen TVT im symptomatischen Bein $(\mathrm{OR}=35,4$; $p<0,0001)$, einer isolierten MVT $(O R=7,4 ; p<0,0001)$ und bei im Krankenhaus erworbener TVT $(O R=2,9 ; p<0,0001)$.

Schlussfolgerung: Die gefundene hohe Rate bilateraler Phlebothrombosen legt nahe, bei Verdacht auf TVT beide Beine einer kompletten sonographischen Diagnostik zu unterziehen.
PS32

Verlust der Lungenfunktion und Diffusionskapazität bei Alpha-1-Antitrypsinmagel

Nikolas Bernhard'; Dr. Sebastian Fähndrich'; Priv.-Doz Philipp M. Lepper'; Prof. Dr. Claus Vogelmeier ${ }^{2}$; Martina Seibert'; Prof. Dr. Stefan Wagenpfeil ${ }^{3}$; Prof. Dr. Robert Bals

${ }^{1}$ Universitätsklinikum des Saarlandes, Klinik für Innere Medizin V; ${ }^{2}$ Department of Medicine, Pulmonary and Critical Care Medicine, University Medical Center Giessen and Marburg, Philipps-University Marburg, Germany, Member of the German Center for Lung Research (DZL); ${ }^{3}$ Institute of Medical Biometry, Epidemiology and Medical Informatics, Saarland University, Campus Homburg

Einleitung: Der Alpha-1- Antitrypsin-Mangel (AATD) wird autosomal-kodominant vererbt. Durch gestörte Neutralisierung der neutrophilen Elastase haben Betroffene ein erhöhtes Risiko, eine COPD und ein Lungenemphysem zu entwickeln. Der jährliche Verlust an Lungenfunktion (FEV1) und Diffusionskapazität (DLCO) gelten als Prädiktoren für Morbidität und Mortalität. Unsere Analyse des deutschen Registers für Alpha-1-Antitrypsin Mangel (gAATR) setzte sich zum Ziel zu untersuchen, welche Faktoren Einfluss auf den Verlust an Lungenfunktion und Diffusionskapazität über die Jahre haben.

Methoden: Anhand der Daten des Daten des gAATR wurde mit generalisierten linearen Modellen (multiple linear generalized estimating equations models /GEE-models) longitudinal der jährliche Verlust der FEV $1(n=100)$ und DLCO $(n=116)$ in Relation zu folgenden Parametern erfasst: Geschlecht, Alter, body mass index (BMI), Nikotinkonsum, berufliche Staubbelastung, Lebensqualität (St. George's Respiratory Questionnaire (SGRQ) score), Basis-FEV1/DLCO, AAT-Serumspiegel, Exazerbationsrate, Steroidgebauch und Zeitdauer zwischen Beginn der Nikotinkarenz und initialer Lungenfunktion.

Ergebnisse: Der Verlust der FEV 1 betrug $-55.22 \pm 169.28 \mathrm{ml} / \mathrm{Jahr}$ und die Verschlechterung der Diffusionskapazität betrug $-0.17 \pm 0.71 \mathrm{mmol} /$ $\mathrm{min} / \mathrm{kPa} / \mathrm{Jahr}$. Eine schnellere Verschlechterung der FEV 1 war mit beruflicher Staubbelastung $(p=0.026)$, Zeitdauer zwischen Beginn der Nikotinkarenz und initialen Lungenfunktion $(p=0.008)$, Basis- FEV1 $(p=0.003)$, jährlicher Exazerbationsrate $(p=0.003)$ und vermehrten Steroidgebrauch $(p=0.004)$ assoziiert. Ein beschleunigter Verlust der DLCO war mit einer deutlich verschlechterten Lebensqualität (SGRQ) assoziiert $(p=0.039)$. Schlussfolgerung: Der jährliche Verlust der FEV 1 ist abhängig von Exazerbationsrate, Nikotinkonsum und beruflicher Staubexposition.

\section{PS33}

Functional status in relation to multimorbidity and impaired kidney function - Results from the Berlin Aging Study II (BASE-II) Dr. Maximilian König'; Prof. Dr. Maik Gollasch²; Dr. Nikolaus Buchmann"; Dr. Dominik Spira'; PD Dr. Kristina Norman';

Prof. Dr. med. Elisabeth Steinhagen-Thiessen'; PD Dr. Ilja Demuth ${ }^{1}$

${ }^{1}$ Charité - Universitätsmedizin Berlin; ${ }^{2}$ Max-Delbrück-Centrum für Molekulare Medizin (MDC)

Background: Reduced kidney function is highly prevalent in the old and has been associated with unsuccessful aging. As chronic kidney disease (CKD) hardly ever occurs isolatedly, the concomitance of reduced glomerular filtration rate (GFR) with multimorbidity, defined as the presence of two or more chronic illnesses, demands attention and has not been sufficiently taken into account in prior studies. To note, most elderly people with reduced GFR have only CKD stage $3 a$ (GFR $45-59 \mathrm{~mL} / \mathrm{min} / 1.73 \mathrm{m2}$ ) and there is an ongoing debate whether the universal threshold of $<6 \mathrm{oml} / \mathrm{min} / 1.73 \mathrm{~m} 2$ is adequate also in the elderly, or should be lower (e.g. $<45 \mathrm{ml} / \mathrm{min} 1.73 \mathrm{m2}$ ). In this context, it remains unclear if there are relevant implications of mild-to-modera- 
tely decreased GFR not only on cardiovascular risk and disease progression but also on physical functioning and mobility.

Objective: To examine the association between reduced kidney function, multimorbidity and physical functioning/mobility as assessed by simple and validated tests, such as the "Tinetti-test" and the "Timed up and Go" (TUG) for mobility as well as the Barthel-index and the Lawton/Brody scale, measuring self-reported impairment in (instrumental) activities of daily living (ADL).

Methods: cross-sectional analysis of the baseline cohort of the Berlin Aging Study II. 1498 participants with complete data were screened for multimorbidity and CKD. Estimated GFR was calculated according to the Full Age Spectrum (FAS) formula and CKD was defined as eGFR $<60 \mathrm{ml} / \mathrm{min} / 1.73 \mathrm{~m} 2$. Multimorbidity (MM) was defined as a count of $>=2$ in a morbidity index (MI), which largely reflects the categories of the established Charlson score. All participants were subjected to comprehensive geriatric assessment, including ADL, IADL, Tinetti and TUG.

Results: The mean age was $68.7 \pm 3.7$ (SD) years, $51.4 \%$ were women. $18.9 \% \mathrm{had}$ an eGFR of $45-49$ and $1.5 \%$ had a GFR $<45 \mathrm{ml} / \mathrm{min} / 1.73 \mathrm{m2}$. The mean Ml count was $1.3 \pm 1.3$ (SD). In univariate analyses, considering mean scores as well as cutoffs, participants with CKD showed significant change for the worse in the Tinetti ( 27.4 Vs. 27.6, $p=0.02$ ) as well as in the TUG (8.3 vs. $7.8 \mathrm{sec}, \mathrm{p}<0.001)$ test. To note, also CKD on top of multimorbidity was associated with impaired functional mobility (Tinetti: 27.6 vs. 27.0, $p=0.02$; TUG: 8.8 vs. 8.0, $p=0.01$ ). Logistic regression, using validated cutoffs, confirmed that CKD was independently associated with impaired functional mobility, both in the Tinetti (OR 2.4, 95\% Cl 1.1-5.5) and in the TUG (OR 2.4, 95\% Cl 1.1-5.7). Notably, ADLs/ IADLs resulted to be unaffected by decreased kidney function.

Conclusion: Mild-to-moderately decreased kidney function is independently associated with a higher risk (as much as 2.5 -fold) of functional mobility impairment. Thus, even early stage CKD seems to preclude successful aging.

\section{PS34 \\ Liver metastases of pancreatic cancer: \\ The interplay of metabolic enzymes and cancer stem cell properties in the context of the hepatic microenvironment Simon Stegner'; Jann Buttlar ${ }^{2}$; Fabrice Viol'; Lennart Lenk²; Hendrike Knaack²; Sascha Rahn²; Alexander Fabian²; Prof. Dr. Wolfgang Mikulits ${ }^{3}$; Prof. Dr. Heiner Schäfer²; Dr. Ole Helm²; Prof. Dr. Susanne Sebens ${ }^{2}$ \\ ${ }^{1}$ UKSH Campus Kiel, Institut für Experimentelle Tumorforschung; ${ }^{2}$ UKSH, Campus Kiel, Institut für Experimentelle Tumorforschung; ${ }^{3}$ Institut für Krebsforschung, Medizinische Universität Wien}

Pancreatic ductal adenocarcinoma (PDAC) has a poor 5-year-survival-rate of $8 \%$ and ranks 4 th in the order of fatal tumor diseases. At time point of diagnosis $80 \%$ of PDAC-patients have an advanced tumor stage often with liver metastases. Metabolic reprogramming is regarded as a hallmark of cancer essentially driving tumor development. Cancer stem cells (CSC) are supposed to be important for initiation of primary tumors and metastases, too. However, our knowledge is still limited on how metabolic alterations impact on CSC properties and vice versa, particularly in the metastatic context. Thus, this study aims at investigating the influence of the hepatic microenvironment -and its inflammatory status- on metabolism-associated enzymes and CSC characteristics of pancreatic ductal epithelial cells (PDEC). Therefore, the two PDEC cell lines $\mathrm{H} 6 \mathrm{c} 7-\mathrm{kras}$ and Panc1 were indirectly cocultured with hepatic stellate cells (HSC), modelling the physiological liver microenvironment, or hepatic myofibroblasts (HMF), representing an inflamed liver microenvironment. To further investigate the mutual influence of CSC characteristics and metabolic alterations on PDEC behavior, siRNA mediated knockdown of CSC markers and metabolic enzymes was performed before coculture experiments. Expression of the CSC markers Nanog and Nestin as well as the clonogenic potential were higher in PDEC cocultured with HSC compared to coculture with HMF. Moreover, HSC cocultured PDEC were characterized by elevated expression of the succinate-dehydrogenase- $\mathrm{B}(\mathrm{SDHB})$, a key enzyme of the citric cycle and respiratory chain, while HMF cocultured cells exhibited higher expression of the glucose-6-phosphate-dehydrogenase (G6PD), a key enzyme of the pentose phosphate way. Knockdown of SDHB resulted in higher cell numbers, an increased clonogenic potential along with an increased expression of Nanog and Nestin in HSC cocultured PDEC. This was accompanied by a clear increase in G6PD expression. In HMF cocultured PDEC, suppression of G6PD resulted in an increased expression of SDHB and analogous effects with respect to elevation of cell numbers and expression of Nanog and Nestin. However, the clonogenic potential remained unaltered. Suppression of Nanog caused higher SDHB expression along with lower expression of G6PD in PDEC under both coculture conditions. Furthermore, knockdown of either CSC marker decreased cell numbers and the clonogenic potential of PDEC in which suppression of Nanog showed the strongest inhibitory effects on colony formation. Overall, these data suggest on the one hand a causal relation between elevated G6PD expression and a gain of CSC characteristics in PDEC within an inflamed liver microenvironment. On the other hand, these findings support the view that SDHB acts as tumor suppressor in a physiological liver milieu. Further studies are urgently needed to unravel the complex interplay between metabolic alterations and CSC properties and how this promotes liver metastasis of PDAC.

\section{PS35}

Expression of ATF3-splicing variants under control of Nrf2 contributes to the shaping of metabolic compartmentalization and symbiosis of pancreatic ductal adenocarcinoma cells

Lisa Dinges; Lisa Hessse; Nourhane Ammar; Prof. Dr. Alexander Arlt; Prof. Dr. Christoph Röcken; Prof. Dr. Susanne Sebens; Prof. Dr. Heiner Schäfer

Background: Pancreatic ductal adenocarcinoma (PDAC) is a highly heterogeneous disease, including different metabolic phenotypes. A hallmark of this heterogeneity is the metabolic compartmentalization/symbiosis adopting glycolytic "Warburg" and nonglycolytic "reverse Warburg" phenotypes. Recent studies revealed an association of the reverse Warburg phenotype with greater malignancy of cancer cells. Important drivers of such metabolic alterations might be the stress responsive transcription factors $\mathrm{Nrf}_{2}$ and $\mathrm{ATF}_{3}$, both exhibiting dichotomous effects in tumorigenesis. The present study investigated the role of $\mathrm{Nrf}_{2}$ and its impact on $\mathrm{ATF}_{3}$ splicing variants in the shaping of PDAC cell metabolism.

Methods: Isoform specific qPCR- and westernblot analysis to selectively detect ATF3, ?Zip or ?Zip2 expression. Generation of variant specific siRNA and cloning of ATF3 variants (full length, ?Zip, ?Zip2) to transfect pancreatic cell lines. Expression analysis of $\mathrm{MCT}_{1} / \mathrm{MCT}_{4}$ lactate $\mathrm{im}$ /exporters and LDHB by real time PCR and westernblot. 14C-Lactate and chemiluminescent glucose uptake-assays. MCT1-promoter luciferase assays and immunohistochemistry with human PDAC tissue for detection of ATF3 or ?Zip2.

Results: Panc1, $\mathrm{T}_{3} \mathrm{M}_{4}$, MiaPaca2 pancreatic cancer and nonmalignant $\mathrm{H} 6 \mathrm{C} 7$ pancreatic duct cells revealed differential expression of ATF3, ?Zip or ?Zip2 that was paralleled by differential MCT1 and $\mathrm{MCT}_{4}$ expression. Nrf2 activation strongly induced ?Zip2 and MCT1 expression in $\mathrm{H} 6 \mathrm{C} 7$ and Panc1 cells. Isotype specific knock down of ATF3 increased MCT1 expression, whereas knock down of ?Zip2 led to a reduced expression of $\mathrm{MCT}_{1}$. By contrast, $\mathrm{MCT}_{4}$ expression was increased by the knock-down of both ATF3 variants. When overexpressed in Panc1 
or MiaPaca2 cells, ATF3 led to a decreased and ?Zip2 to an increased $M C T_{1}$ expression. Luciferase assays with the MCT1 promoter confirmed the inducing effect by ?Zip2, which is due to the release from transcriptional repression by ATF3 via a CREB binding site. As shown by ${ }_{14 C}$-lactate uptake assay, MCT1 driven lactate incorporation in pancreatic duct cells is under control of the ATF3/ ?Zip2 expression ratio which is mainly shifted to predominant ?Zip2 expression by Nrf2. Conversely, glucose uptake linked to GLUT1 expression is positively and negatively affected by ATF3 and ?Zip2, respectively. Immunohistochemical analysis detected co-expression of MCT1, Nrf2 and ?Zip2 in PDAC tissues, whereas $\mathrm{ATF}_{3}$ is reciprocally expressed.

Discussion: The Nrf2/ATF3-?Zip2 axis has an essential role in metabolic symbiosis of PDAC cells by increasing lactate uptake through MCT1. Thereby, these PDAC cells adopt a reverse Warburg phenotype and are capable to share a metabolic flux with lactate-releasing/Warburg phenotype cells (e.g. cancer associated fibroblasts). Thus, interfering with this axis (e.g. by Nrf2 inhibitors like trigonelline) and/or shutting down metabolic symbiosis (e.g. by MCT1 inhibitors like AZD3965) will offer new options for cancer therapy.

\section{PS36 \\ Prädiktoren für einen ungünstigen Verlauf bei ischämischer Kolitis - Eine retrospektive Analyse von 46 internistischen Patienten \\ Dr. Jessica Rüddel'; Daniel Thomas-Rüddel'; Dr. Martin Bürger'; Priv.-Doz Carsten Schmidt'; Prof. Dr. Andreas Stallmach ${ }^{1}$ \\ ${ }^{1}$ Friedrich-Schiller-Universität, Universitätsklinikum Jena; ${ }^{2}$ Universitätskli- nikum Jena der Friedr. Schiller Universität}

Einleitung: Intestinale Ischämien sind häufiger als bisher angenommen und stellen häufig eine lebensgefährliche Komplikation oder Begleiterkrankung dar. Die ischämische Kolitis tritt häufig im postoperativen Verlauf, meist nach kardiochirurgischen Eingriffen, auf. Hiervon abzugrenzen ist die ischämische Kolitis, welche auch ohne erkennbaren Auslöser auftritt und meist bei älteren Patienten mit Komorbiditäten zu beobachten ist. Problematisch ist neben der nicht standardisierten Diagnostik und Therapie die hohe Mortalität mit bis zu 25\%. Vor diesem Hintergrund analysierten wir alle Patienten, welche zwischen 2009 und 2014 aufgrund einer ischämischen Kolitis im Universitätsklinikum Jena in der Klinik für Innere Medizin IV stationär aufgenommen wurden. Von besonderem Interesse waren mögliche Prädiktoren für einen ungünstigen Verlauf.

Ergebnisse: In den analysierten 5 Jahren wurden 46 Patienten aufgrund einer ischämischen Kolitis behandelt. Die Patienten waren zum Zeitpunkt der Diagnose im Schnitt 78 Jahre alt und signifikant häufiger weiblich $(76 \%)$ als männlich $(24 \%)(p=0,001)$. Die häufigsten Symptome waren rektale Blutabgänge (70\%), abdominelle Schmerzen (57\%) und Durchfälle (35\%), seltener mit abdomineller Abwehrspannung (13\%). 95\% der Patienten hatten eine bekannte arterielle Hypertonie, $40 \%$ eine arteriosklerotische Erkrankung. Das Befallsmuster war überwiegend linksseitig (Rektum 32\%, Sigma 70\%, C. descendens $43 \%$, C. transversum $17 \%$ ) und seltener im rechtsseitigen Kolon (15\%). Die Therapie erfolgte mittels Antikoagulation oder TAH (82\%), Antibiose (68\%) oder antiinflammatorisch mittels Mesalazin oder PrednisoIon (42\%). Eine chirurgische Therapie war bei $20 \%$ der Patient notwendig. Insgesamt bestand eine Krankenhausmortalität von 10\%. 18\% der Patienten wurden bis 2016 aufgrund eines Rezidivs im gleichen Krankenhaus behandelt. Operation und Tod wurden als kombinierter Endpunkt, das Auftreten eines Rezidivs als weiterer Endpunkt betrachtet. Als relevanter Prädiktor für eine operative Therapie oder Tod erwies sich die abdominelle Abwehrspannung $(p=0,009)$ und eine Leukozytose $(p=0,011)$ bei Aufnahme. Auch hatten die Patienten, welche nach initial internistischer Aufnahme einer operativen Therapie bedurften, eine signifikant höhere Mortalität $(p=0,009)$. Bezüglich des Auftreten eines Rezidives war die arterielle Hypotonie $(p=0,019)$ und ein erhöhter CRP-Wert $(p=0,04)$ bei Aufnahme signifikant und der rechtsseitige Befall im Trend prädiktiv $(p=0,057)$. Es zeigte sich kein Unterschied bezüglich der Endpunkte hinsichtlich einer Therapie mit Prednisolon.

Diskussion: In einer typischen internistischen Kohorte von 46 Patienten mit ischämischer Kolitis konnten als Prädiktoren für einen ungünstigen Verlauf (Rezidiv, OP oder Tod) die abdominelle Abwehrspannung, eine Leukozytose und erhöhtes $\mathrm{CrP}$ bei Aufnahme sowie der rechtsseitige Befall gezeigt werden.

\section{PS37}

4-PBA, ein synthetisches Chaperon, aktiviert den Transkriptionsfaktor ATF6 im murinen HBV-Modell

Dipl.-Bio. Katrin Maria Baier'; Dr. Yuri Churin; Franziska Schneider; Alica Köppel; Dagmar Leder; Annette Tschuschner; Dr. Martin Roderfeld; Prof. Dr. med. Elke Roeb

${ }^{1}$ UKGM GmbH/ Med. I

Einleitung: Das synthetische Chaperon 4-PBA (Phenylbuttersäure) bewirkt in verschiedenen Modellen für Protein-Aggregationskrankheiten die Auflösung zellschädigender Aggregate (Wiley 2011). Ein direkter, intrinsischer Mechanismus der Zellschädigung bei der HBV Infektion beruht auf der Akkumulation der HBV-Hüllproteine (HBs) im Endoplasmatischen Retikulum (ER) der Hepatozyten, was zu ER-Stress und Apoptose führen kann (Churin 2014). ATF6 ist ein ER-Stress regulierender Transkriptionsfaktor und führt zur erhöhten Bildung von Chaperonen. Das Ziel unserer Studie war die Untersuchung der Wirkung von 4-PBA auf die Aktivierung von ATF6 im murinen HBV-Modell.

Methoden: Eine Expressionsinduktion von HBs-Antigen mit Doxycyclin erfolgte in stabil transfizierten $\mathrm{HuH}_{7}$ und $\mathrm{NIH}_{3} \mathrm{~T}_{3}$ Zellen (HBs überexprimierende Zellen). Im Anschluss wurde eine in vitro Behandlung mit PBA durchgeführt und die Zelllysate immunhistochemisch, mittels Western Blot und qRT-PCR analysiert. In vivo wurden HBs-transgene Mäuse $\mathrm{C}_{57} \mathrm{BL} / 6 \mathrm{~J}-\mathrm{tg}(\mathrm{Alb} 1 \mathrm{HBV}$ )44Bri/J über einen Zeitraum von 1-8 Wochen kontinuierlich mit PBA (im Trinkwasser) behandelt. Das Tiermodell wurde mittels Immunhistochemie, mRNA-Array, qRT-PCR (u. a. Signal Transduction Pathway Finder), Proteome Profiler und Western Blot (u. a. Subcellular Fractionation) analysiert.

Ergebnisse: Immunhistochemische Färbungen stabil transfizierter $\mathrm{HuH}_{7}$ und $\mathrm{NIH}_{3} \mathrm{~T}_{3}$ Zellen verdeutlichen, dass nach PBA Zugabe die Lokalisation des Transgens beeinflusst wird, die intrazellulären Aggregate fein verteilt auftreten und teilweise aufgelöst werden. Im transgenen Mausmodell zeigte die Expression der HBV-Hüllproteine unter PBA-Gabe ebenfalls ein verändertes intrazelluläres Aggregationsmuster mit konsekutiver Feinverteilung des Transgens HBs. PBA verstärkt weiterhin die Expression von ATF6 und durch dessen nukleäre Translokation die Expression von Akut-Phase-Proteinen (Serum Amyloid, Lipocalin, Metallothionein). Schlussfolgerungen Die Zugabe des synthetischen Chaperons PBA bewirkt in vitro und in vivo eine Auflösung der HBV-Hüllproteinaggregate und eine damit assoziierte Verringerung von Zellstress. Die Akkumulation von falsch gefalteten Proteinen im ER führt zur proteolytischen Spaltung von ATF6. Der cytosolische Teil von ATF6 wird in den Zellkern transportiert und wirkt als Transkriptionsfaktor. Eine gezielte Immunmodulation mit PBA offenbart neue Perspektiven für die HBV-Therapie. Die Ergebnisse unserer Studie tragen zum besseren Verständnis der zellulären Pathophysiologie von HBV Oberflächenproteinen bei und zeigen potentielle Wege zur Entwicklung neuer Therapieverfahren auf. 


\section{PS38}

Pyoderma gangrenosum als paraneoplastisches Syndrom im Nierenzellkarzinom: Fallbericht und Literaturübersicht Dr. med. Ioana Duca'; Dr. med. Nicolae Alin Nicula;

Prof. Dr. med. Dan Lucian Dumitrascu

${ }^{1}$ II.Medizinische Klinik, Str.Clinicilor ${ }^{2-4}$, Cluj-Napoca, Rumaenien

Ziel: Paraneoplastische Pyoderma gangrenosum erscheint in ungefähr $7 \%$ der Fälle, insbesonders im hämatologischen Bereich; nur 3 Studien haben bis jetzt über den Zusammenhang mit Nierenzellkarzinom berichtet.

Material und Methoden: Wir stellen den Fall eines 6o.jährigen Patienten vor, bekannt mit Hypertonie, Vorhofflimmern, dilatativer Kardiomiopathie, Herzinsuffizienz, welcher wegen Dyspnoe, Fieber, langanhaltenden schmerzhaften therapierefraktaren Ulcera in der rechten Leiste eingewiesen wurde. Es wurden folgende Untersuchungen durchgeführt: klinischer Befund, Laborwerte, Ultraschall, CT, dermatologische Beratung, Punktion-Biopsie der Ulcera und Histologie.

Ergebnisse: Die ärztliche Untersuchung stellte Adipositas, erhöhten Blutdruck 170/90mm Hg, komplette Arrythmie, Dyspnoe, Ödeme, schmerzhafte Ulcera der rechten Leiste begrenzt von einem rötlichem Rand fest. Der Laborbefund zeigte ein hohes Entzündungssyndrom (BKS 70/h, CRP 16mg/dl), leicht erhöhtes Kreatinin (1.33mg/dl), Anämie $(\mathrm{Hb} 10.8 \mathrm{~g} / \mathrm{dl})$, Glukoseintoleranz, negative Serologie für Hepatitis B/C und HIV, negative Immunologie (ANA, $\lg A$, $\lg M, \lg G, C_{3}, C_{4}$ ), Leukoziturie, negative Urokultur und keine Hämaturie. Ultraschall wies ein vaskularisierter Tumor am oberen Nierenpol links mit zystischen Anteilen, und eine inhomogene Leberstruktur mit V.a. Lebermetastasen und Riedellappen auf. CT schilderte einen $7.3 \mathrm{~cm}$ grossen Nierentumor, welcher inhomogen sowohl vor wie auch nach Kontrastmittelgabe schien. Bakteriologische Untersuchung der Leistenulcera wies Pseudomonas aeruginosa und Candida spp auf, Antibiotika (Ciprofloxacin), und Antimykotika fuehrte aber nicht zu deren Heilung; somit wurde die Histologie, welche Pyoderma gangrenosum nachwies, erforderlich. Orale Kortikoidtherapie gefolgt von Tumorresektion führte erst nach 2 Monaten zur Heilung der Ulcera. Histologisch wurde der Nierentumor als Adenokarzinom eingeordnet.

Schlussfolgerung: Patienten mit atypischen antibiotikarefraktaren Hautulcera sollten sich immer einen Tumorscreening unterzogen werden, auch wenn es keinen klinischen Hinweis für ein organspezifisches Bild gibt.

\section{Personalisierte Pharmakotherapie 1}

\section{PS39 \\ Early Clinical Trial Unit (ECTU) and entire Clinical Trial (CT) activities in hematology\&oncology (H\&O) departments supported by the Clinical Cancer Research (CCR-) Group Freiburg and Comprehensive Cancer Center Freiburg (CCCF) \\ Irina Surlan; Dr. Ulrike Kohlweyer; Dr. Martin Köhler; \\ Prof. Dr. med. Justus Duyster; PD Dr. med. habil. Lena Illert; \\ Prof. Dr. med. Monika Engelhardt \\ University of Freiburg Medical Center, Hematology, Oncology \& Stem Cell transplantation; Comprehensive Cancer Center Freiburg (CCCF), Clinical \\ Trials Center (CTC), Germany}

Introduction: $\mathrm{CTS}$ in $\mathrm{H} \& \mathrm{O}$ are broadly performed due to enormous drug advances and abundant medical progress, making the organisation and logistics of CTs, most of which test novel agents within AMG-restrains, a daily challenge. Increasing numbers of early CTs have been drawn to newly established ECTUs and CCCs.
Methods: We report of our organization structure and support of CTs through our GCP-, ECTU- and CCCF-team.

Results: Our team is responsible for 1. cancer CTs conducted in $\mathrm{H} \& \mathrm{O}$, 2. documentation of patients (pts) within (and outside) CTs using an electronic tumor base documentation (Carat+) system and 3. chemotherapy (CTx) support and logistics. In close cooperation with PIs, our CT office coordinates all essential CT-tasks: from first contacts (with sponsors/CROs, ethic committees, BfArM, PEl, CTC), to the evaluation of CTs of interest, logistics and CRF-documentation. In order to estimate highly realistic pt recruitment numbers, we employ our Carat+ system: this includes demographics, cancer histologies, all diagnoses (including comorbidities), prior CTx-regimens, SAEs and progression and survival data. Another highly valuable CT-support tool is our protocol-study-and-review-board meeting that discusses newly proposed CTs with our Pls, e.g. pro and cons of CTs and feasibility. Relevant aspects, such as sufficient pt numbers and regulatory and financial aspects are also eagerly discussed therein. In order to enhance pt recruitment into CTs, we support various effective CT-recruitment strategies, e.g. educational programs, ProTrials, cross-linkage to CTx-management, intra-/internet and CT-search programs (e.g.QuickQueck). Entity-specific tumorboards are another useful tool to efficiently enrol pts into active CTs. Pts' response to our CCCF-ECTU has been exceptionally good: organization, study nurse and medical support and entire study assistance were judged as excellently performed. The increase in phase I early CTs has been accompanied with an increase in pt enrollment and impressive pt numbers being treated per month within this successful unit.

Conclusions: The ECTU supports our expertise in clinical research that is of utmost importance for new drug development. In times of increasingly dense daily physicians' schedules and with enormous CT-logistics today, our GCP- and CCCF-ECTU-structure is highly valuable for eager trial performance, our results possibly serving as an useful example for other ECTU- and CCCs.

\section{PS40}

Chemotherapy applied prior to hemodialysis in patients with pancreatic cancer and end stage renal disease

Dr. med. Alexander Hann; Evelyn Nosalski; Frieder Keller

Universitätsklinikum Ulm

Background: Pancreatic ductal adenocarcinoma (PDAC) is associated with a poor prognosis. New survival prolonging chemotherapy strategies are incorporated in daily routine. Although the incidence of renal insufficiency and end stage renal disease (ESRD) are increasing, most studies exclude such patients in the evaluation of chemotherapies. The purpose of this study is to provide guidance for the clinician to use chemotherapy in a patient with pancreatic cancer on hemodialysis (HD). Additionally a thorough review of the literature compares the traditional use of chemotherapy application after HD to the new concept of chemotherapy shortly prior to HD. This new concept mimics near to normal renal function for dialyzable substances.

Methods: We retrospectively identified all PubMed listed studies describing patients with ESRD receiving chemotherapy until October 2016. We selected traditional (gemcitabine, $5 \mathrm{FU}$, capecitabine, oxaliplatin, irinotecan, docetaxel) and novel (erlotinib, sunitinib, S-1, afatinib, checkpointinhibitors) chemotherapeutic agents used in the therapy of PDAC. Time of chemotherapy application relative to HD, toxicity and pharmacokinetic measurements were assed.

Results: We identified 59 studies describing 132 patients with ESRD receiving chemotherapy. The application directly before HD was successfully used for the dialyzable substances gemcitabine, $5 \mathrm{FU}$, capecitabine, oxaliplatin, irinotecan and S-1. Conclusion: Chemotherapy in 
patients with PDAC is feasible and can be improved due to the application of chemotherapeutic agents directly before HD.

\section{PS41 \\ Proprotein convertase subtilisin/kexin type 9 Antikörper (PCSK9-I) in der Behandlung von kardiovaskulären Hochrisikopatienten in der klinischen Praxis - Neue Daten zu Sicherheit und Effektivität \\ Dr. Tim Hollstein'; Thomas Grenkowitz $z^{2}$; Prof. Dr. Hubert Scharnagl ${ }^{3}$; Prof. Dr. med. Winfried März?; Prof. Dr. Elisabeth Steinhagen-Thiessen;; Dr. Ursula Kassner ${ }^{1}$ \\ ${ }^{1}$ Charité Universitätsmedizin Berlin; ${ }^{2}$ Charité - Universitätsmedizin Berlin; ${ }^{3}$ Medizinische Universitätsklinik Graz; ${ }^{4}$ SYNLAB Holding Deutschland GmbH}

Ziel: Vor einem Jahr wurden PCSK9-I zur Behandlung der therapierefraktären Hypercholesterinämie zugelassen. Ziel dieser Studie ist die Evaluierung von Sicherheit und Effektivität der PCSKg-I im klinischen Alltag, insbesondere bei Patientengruppen mit erhöhtem Lipoprotein(a) $[\mathrm{Lp}(\mathrm{a})]$ und Diabetikern.

Methoden: 290 Patienten mit hohem und sehr hohem kardiovaskulären Risiko gemäß der European Society of Cardiology (ESC) Leitlinien, die die LDL-Cholesterin Zielwerte unter maximal tolerierter lipidsenkender Therapie nicht erreichen oder statinintolerant sind, wurden zur Behandlung mit Alirocumab oder Evolocumab (150 mg bzw. 140 $\mathrm{mg}$ alle 2 Wochen) zugelassen. Klinisch indizierte Blutuntersuchungen wurden vor und einen Monat nach Behandlungsbeginn mit PCSK9-I durchgeführt.

Ergebnisse: Die Behandlung mit PCSKg-I senkte Gesamt- und LDL-Cholesterin um 38 bzw. 52 Prozent. Triglyceride wurden um 26 Prozent gesenkt, während HDL-Cholesterin um $14,5 \%$ anstieg. Die LDL-Cholesterin Senkung korrelierte mit dem Baseline-LDL-Cholesterin und der Lp(a) Reduktion bei Patienten mit erhöhtem Lp(a). Diabetiker mit erhöhtem $\mathrm{HbA}$ cc zeigten eine stärkere Reduktion der Triglyceride. Interessanterweise führte die Therapie zu einem leichten CRP-Anstieg um $1 \mathrm{mg} / \mathrm{l}$, weiterhin wurde eine minimale Senkung von Hämoglobin und Erythrozyten beobachtet.

Zusammenfassung: Der geringe CRP-Anstieg nach einmonatiger Therapie bei Patienten ohne bekannte Infektion ist bisher nicht kausa erklärt und bedarf weiterer Beobachtung. Diabetiker scheinen von einer erhöhten Triglycerid-Reduktion aufgrund verringerter VLDL und verringerter Triglyceride in LDL-Partikeln zu profitieren. Die PCSK9-I Behandlung scheint Hämoglobin und Erythrozyten minimal zu senken, was weiterer Beobachtung bedarf.

\section{PS42}

Treatment of stable angina with a fixed-dose combination of ivabradine and metoprolol: first data from clinical practice Dr. med. Dimitar Divchev'; Dr. Georg Stöckl

1 Universitätsklinikum Gießen und Marburg, Standort Marburg

Objectives: The symptomatic benefits of the selective heart rate reducing agent ivabradine, beta blockers and also the free combination of both in the treatment of stable angina pectoris have been proven in clinical trials. However, data is still lacking on use of a fixed-dose combination (FDC) therapy with these drugs. The current study aimed to evaluate the effectiveness of the first FDC formulation of ivabradine and the beta blocker metoprolol in clinical practice. The presented data are preliminary results from this study.

Methods: In this analysis from a large prospective, multicenter, non-interventional cohort study 610 patients with stable angina pectoris were included. Patients received a FDC of ivabradine and metoprolol bid for 4 months, in addition to existing cardiovascular standard therapy. Among other parameters, resting heart rate (HR), the number of angina attacks, short acting nitrate consumption, co-medication, severity of symptoms (by CCS score) and tolerability were documented. Adherence to therapy was assessed by use of a modified Morisky scale questionnaire. A descriptive statistical analysis of all data was performed.

Results: Mean age of the cohort was 66.3 years, $42 \%$ were $? 70$ years, $10 \% ? 80$ years, $63 \%$ male, $31 \%$ had a history of myocardial infarction, $61 \%$ of $\mathrm{PCl}$ and/or CABG, mean BMI was $28.5 .86 \%$ of all patients were already treated with a free combination of ivabradine (mean dose 9.9 $\mathrm{mg} / \mathrm{d}$ ) and beta-blockers (metoprolol $94 \%$, mean dose $92.6 \mathrm{mg} / \mathrm{d}$ ) before inclusion. Other concomitant standard medication consisted of e.g. aspirin $67 \%$, statins $71 \%$, ACEI/ARB $77 \%$, diuretics 33\%, long acting nitrates $6 \%$, calcium antagonists $16 \%$, molsidomine $5 \%$, ranolazine $5 \%$. Most prevalent comorbidities were hypertension (87\%), hyperlipidaemia (65\%), diabetes (34\%) and COPD/asthma (20\%). After 4 months, additional treatment with the FDC was associated with a significant reduction in mean HR by $9.8 \mathrm{bpm}$. Proportion of patients with ?1 angina attacks/week substantially decreased from $37 \%$ to $6 \%$, accompanied by a comparable decline in use of short-acting nitrates. Patients in CCS class 1 increased from $26 \%$ to $63 \%$, while proportion in CCS class 3 declined from 19\% to 6\%. " "Complete adherence "' (defined as absence of any adherence-related problems reported in questionnaire) was achieved in $58 \%$ of patients after 4 months, compared to $34 \%$ at baseline ( $p<0.001$ for all changes from baseline). Tolerability of the FDC was rated as very good/good in $74 \% / 25 \%$ of patients.

Conclusion: In this study cohort of stable angina patients in a real-life setting, showing already intense anti-anginal pre-treatment at baseline, an ivabradine/metoprolol FDC additionally reduced HR, angina symptoms and nitrate consumption. Exercise capacity was improved as reflected by a significant shift of patients to lower CCS classes. These positive effects may be associated with the marked improvement in treatment adherence that could be observed with the FDC formulation during the study period.

\section{PS43}

Organspezifische Immunsuppression ohne systemische Nebenwirkungen im Nierentransplantationsmodell in der Ratte Dominik Kentrup'; Katharina Schütte-Nütgen'; Helga Pawelski'; Hermann Pavenstädt'; Sven Hermann²; Michael Schäfers²; Gregor Larbig ${ }^{3}$; Armin Kübelbeck ${ }^{3}$; Stefan Reuter ${ }^{1}$

${ }^{1}$ Universitätsklinikum Münster; ${ }^{2}$ European Institute for Molecular Imaging - EIMI, Universitätsklinikum Münster; ${ }^{3}$ Merck KGaA

Zielsetzung: Bedingt durch die systemischen Wirkungen der verabreichten Medikamente, gehen immunsuppressive Therapien mit zum Teil schweren Nebenwirkungen einher. Ziel dieses Projektes war daher die Prüfung einer organspezifischen immunsuppressiven Therapie im allogenen Nierentransplantationsmodell in der Ratte. Verwendet wurde eine modifizierte Version von Prednisolon, welches, gekoppelt an ein Polypeptid, selektiv von der Niere aufgenommen wird, so dass systemische Nebenwirkungen vermieden werden können.

Methoden: Folgende Versuchsgruppen ( $\mathrm{N}=5-6$ pro Gruppe) wurden untersucht: Syngen (Lewis Brown Norway F1 auf Lewis Brown Norway F1) und allogen nierentransplantierte Tiere (Lewis Brown Norway F1 auf Lewis) ohne Immunsuppression, sowie allogen transplantierte Tiere mit immunsuppressiver Therapie in Form von konventionellem oder modifiziertem Prednisolon in zwei verschiedenen Dosierungen ( $4 \mathrm{mg} / \mathrm{kg} / 12 \mathrm{~h}$ oder $16 \mathrm{mg} / \mathrm{kg} / 12 \mathrm{~h}$, i.p). Für die Experimente wurden sowohl ein präventiver Ansatz (kontinuierliche Behandlung ab Transplantation), als auch ein therapeutischer Ansatz (Behandlungbeginn 4 Tage nach Transplantation nach Entstehung einer akuten Abstos- 
sungsreaktion). Die Beurteilung des Behandlungserfolges erfolgte mittels 18F-FDG Positronen-Emissions-Tomographie (PET) an Tag 4 nach Transplantation beim präventiven Ansatz, sowie an Tag 4, 5 und 7 nach Transplantation beim therapeutischen Ansatz, zuzüglich einer histologischen Befundung am letzten Versuchstag ( 4 bzw. 7). Messungen der Blutglukosespiegel wurden durchgeführt, um die systemische Wirkung der Therapie zu beurteilen.

Ergebnisse: Allogen transplantierte Tiere ohne Behandlung zeigten in beiden verfolgten Ansätzen deutliche Zeichen einer Transplantatabstoßung (erhöhte 18F-FDG-Akkumulation, ausgeprägte T-Zell-Infiltration im Transplantat), syngen transplantierte Tiere hingegen nicht. Konventionelles Prednisolon führte nur bei hoher Dosierung zu einer signifikanten Verminderung sowohl des PET Signals als auch der T-Zell-Infiltration in beiden Ansätzen, eine niedrige Dosierung zeigte keine Wirkung. Demgegenüber zeigten die mit modifiziertem Prednisolon behandelten Tiere bereits bei einer niedrigen Dosierung eine signifikante Besserung. Eine Dosissteigerung führte zu einer weiteren Reduktion sowohl des PET Signals, als auch der T-Zell Infiltration. Systemische Effekte in Form erhöhter Blutglukosewerte konnten zudem nur nach Gabe von herkömmlichem Prednisolon beobachtet werden.

Schlussfolgerung: Verglichem mit konventionellem Prednisolon zeigt die Behandlung mit modifiziertem, nierenspezifisch aufgenommenen Prednisolon eine wenigstens gleiche, in Teilen sogar bessere immunsuppressive Wirkung ohne systemische Nebenwirkungen. Eine organspezifische Immunsuppression ist, zumindest im Falle der Niere, möglich.

\section{PS45 \\ Individuelle Prednisolontherapie einer Schwangeren mit Colitis ulzerosa bei häuslicher Selbstmessung von Calprotectin \\ Dr. med. Moritz A. Weiher'; Dr. med. Daniel Mitas; PD Dr. Michael Selgrad; \\ Prof. Dr. med. Martina Müller \\ ${ }^{1}$ Klinikum der Universität Regensburg}

Fall: Aufgrund von erneuten blutigen Diarrhoen von bis zu 2omal pro Tag wurde eine 30-jährige Schwangere aus einem peripheren Krankenhaus zur weiteren Therapie einer bekannten Colitis ulzerosa an unser Haus überwiesen. Die Diagnose der Colitis wurde erstmals 2 Monate zuvor gestellt und es zeigte sich bereits ein steroidabhängiger Verlauf. Die Akuttherapie mit einer körpergewichtsadaptierten Dosis von 7omg Prednisolon zeigte ein gutes Ansprechen. In Rücksprache mit der Patientin wurde aufgrund der fortgeschrittenen Schwangerschaft in der 15. Gestationswoche, der Sorge der Mutter vor Nebenwirkungen einer immunsuppressiven Therapie sowie bei raschem und gutem Ansprechen auf Prednisolon eine Fortsetzung der Glukokortikoidtherapie besprochen. Um die Prednisolondosis so gering wie möglich zu halten und frühzeitig ein Therapieversagen zu entdecken erhielt die Patientin mehrere Testkits für eine häusliche Selbstmessung von Calprotectin aus dem Stuhl. Dabei kamen die QuantOnCal ${ }^{\circledast}$ Testkits der Firma Preventis, Benzheim, zur Anwendung, welche uns freundlicherweise vom Hersteller zur Verfügung gestellt wurden. Damit bestimmte die Patientin in einem regelmäßigen Intervall sowie bei dem subjektiven Gefühl einer Verschlechterung den Calprotectinwert selbst und schickte uns teilweise Proben für die Standard Laborbestimmung mittels ELISA ein. So konnte die Prednisolondosis, außer durch regelmäßige Vorstellung in unserer Ambulanz, auch telefonisch und durch die Patientin selbst angepasst werden. Die Patientin war während der Schwangerschaft in einem subjektiv guten Zustand und hat im November ein gesundes Kind zur Welt gebracht. Man kann retrospektiv eine direkte Korrelation zwischen dem Ansprechen auf die Therapie und dem Calprotectinwert erkennen. Es zeigt sich ein ungefährer Grenzwert von ca. 7,5-10mg Prednisolon pro Tag als minimale remissionserhaltende Dosis. Durch die leichte Verfügbarkeit und das rasche Ergebnis konnte die Thera- pie deutlich früher an die Bedürfnisse angepasst werden als durch die konventionelle ELISA Bestimmung im Labor.

Fazit: Moderne Testverfahren ermöglichen eine rasche und schnelle Therapieüberwachung auch von zu Hause. Viele Patienten sind sehr stark daran interessiert ihre Erkrankung zu überwachen und auch die Therapie auf ein Mindestmaß anzupassen. Besonders in der Schwangerschaft stellen chronische Erkrankungen die Patientinnen und behandelnden Ärzte vor die Schwierigkeit, den Schutz von Mutter und Kind zum einen, aber auch eine ausreichende Therapie zu gewährleisten. Gerade hier ist die Möglichkeit der häuslichen Selbstbestimmungen von Vorteil. Durch das raschere Ergebnis und auch die häufigeren und situationsangepassten Bestimmungen kann eine Therapie besser überwacht und gesteuert werden. Wir konnten in diesem Beispiel durch eine häusliche Selbstmessung von Calprotectin eine Optimierung der Prednisolontherapie zur Behandlung einer Colitis ulzerosa in der Schwangerschaft demonstrieren.

\section{Personalisierte Pharmakotherapie 2}

\section{PS46}

Pharmakogenetische Tests in der personalisierten Medizin:

Ein systematisches Review zur Kosteneffektivität von

pharmakogenetischen Tests bei genotyp-adjustierten Arzneimitteltherapien

Marika Plöthner; Dana Ribbentrop; Jan-Philipp Hartmann; Dr. Martin Frank

Hintergrund: In den letzten Jahren fand ein vermehrter Einsatz von zielgerichteten Therapien statt. Pharmakogenetische Tests (pT) stellen ein essentielles Instrument dar, um die Patientenbehandlung zu steuern und die Wirksamkeit von Medikamenten vor der Applikation zu testen. Dabei können pT sowohl Aussagen über die Resistenz von Arzneimitteln liefern als auch zur Bestimmung von genotyp-basierten Medikamentendosierungen herangezogen werden. In diesem Zusammenhang wird oft die Kosteneffektivität (KE) der pT zu diagnostischen Zwecken diskutiert. Ende 2015 waren in Deutschland bereits 47 Medikamente zur personalisierten Medizin zugelassen. Für 39 von diesen war dabei ein vorheriger pT verpflichtend und für acht empfohlen. Das Ziel der Studie war es, einen Überblick über die KE von pharmakogenetischen Arzneimitteltherapien zu geben und hierbei speziell Studien herauszustellen, welche die Medikamentenapplikation mit und ohne einen vorherigen Test untersuchen.

Methoden: Im November 2015 wurde eine systematische Literaturrecherche in 16 Datenbanken (u.a. Medline, Embase, Cochrane) durchgeführt. Die Suchergebnisse wurden auf den Zeitraum zwischen Januar 2000 bis November 2015 begrenzt. Weiterhin wurden die eingeschlossenen Studien einer Qualitätsbewertung unterzogen, wofür das validierte QHES-Instrument (Quality of Health Economic Studies) verwendet wurde.

Ergebnisse: In der Mehrheit der eingeschlossenen Studien stellt die Anwendung eines pT eine kosteneffektive bzw. kostensparende Behandlungsoption dar. In sieben Studien konnte u.a. aufgrund von Unsicherheiten und Begrenzungen auf spezifische Patientenpopulationen keine deutliche Aussage zur KE oder zu den Kosteneinsparungen getroffen werden. Insgesamt konnte eine hohe Qualität der verfügbaren Evidenz evaluiert werden.

Fazit: PT stellen eine Möglichkeit dar, um die KE von Pharmakotherapien zu verbessern. Die KE von zielgerichteten Therapien hängt von diversen Faktoren, u.a. wie den Kosten, der Prävalenz der Biomarker und der Sensitivität und Spezifität des Tests ab. Eine Vergleichbarkeit der KE-Studien von stratifizierten Medikamententherapien kann nur 
durch eine Definition von nationalen und internationalen Standards für Evaluationsstudien gewährleistet werden.

\section{PS47 \\ ENCORE: Charakterisierung einer Patientenpopulation mit schwerem Asthma mit und ohne eosinophile Entzündung sowie häufigen Exazerbationen anhand multipler PROs (IDEAL) Linda Nelsen; Robert Suruki; Frank Albers; Eric Bradford; Sarah Cockle; Necdet Gunsoy; Ji-Yeon Shin}

Einleitung: Patientenberichtete Outcomes (PRO) helfen die Auswirkungen einer Asthma-Erkrankung zu charakterisieren. Mepolizumab (Anti-IL5) ist eine effektive Therapie bei schwerem Asthma für Patienten mit einer eosinophilen Entzündung und häufigen Exazerbationen in der Vergangenheit. Diese post-hoc-Analyse der IDEAL-Studie vergleicht Patienten mit schwerem Asthma, die geeignet sind für eine Behandlung mit Mepolizumab, mit nicht geeigneten Patienten, unter Berücksichtigung von PROs zu Asthmakontrolle, Symptomen und Gesundheitszustand.

Methoden: IDEAL war eine Querschnitts-Studie in 6 Ländern und schloss Patienten ?12 Jahre mit schwerem Asthma (ATS-/ ERS-Guidelines) unter Behandlung mit hochdosiertem ICS und zusätzlich Controller(n) über ?12 Monate ein. Die Datenerhebung beinhaltete eine Blutprobe, eine Spirometrie und die Erhebung von PROs. Die Eignung für Mepolizumab wurde definiert als ? 2 Exazerbationen, die eine Behandlung mit systemischen Corticosteroiden, einen Notaufnahmebesuch oder eine Hospitalisierung in den letzten 12 Monaten erforderlich machten, und ein Blut-Eosinophilen-Wert ?150 Zellen/ $\mu \mathrm{l}$ bei Studieneinschluss oder ein historischer Messwert ?300/ $\mu \mathrm{l}$. Die Studienteilnehmer absolvierten den Asthma Control Questionnaire (ACQ-5; Skala 0-6), St. George's Respiratory Questionnaire (SGRQ; Skala 0-100), Asthma Quality of Life Questionnaire (AQLQ; Skala 1-7) und Asthma Symptom Utility Index (ASUI; Skala 0-1). Höhere Werte für den ACQ und SGRQ und niedrigere Werte im AQLQ und ASUI deuten auf eine schlechtere Gesundheit hin.

Ergebnisse: 748 Studienteilnehmer wurden in IDEAL eingeschlossen; 670 erfüllten die Analyse-Kriterien. Nach Ausschluss von derzeit mit Omalizumab behandelten Patienten wurden 502 (Durchschnittsalter 50,9 Jahre; 62\% weiblich) eingeschlossen; davon wurden 101 (20,1\%) und $401(79,9 \%)$ als geeignet beziehungsweise nicht geeignet für eine Mepolizumab-Therapie eingestuft. Mepolizumab-geeignete (MG) Patienten hatten eine schlechtere Asthmakontrolle (Mittel=2,64 $[S A=1,42] ; 77 \%$ mit unkontrolliertem Asthma) vs. Nicht-Mepolizumab-geeigneten (NMG) Patienten $(1,95[1,14] ; 64 \%$ mit unkontrolliertem Asthma). Die Werte im SGRQ, AQLQ und ASUI wiesen auf schlechtere Gesundheitsauswirkungen in der MG-Gruppe hin $(53,2[19,4] ; 4,1$ $[1,2] ; 0,55[0,25])$ vs. NMG-Patienten $(39,7[19,4] ; 4,7[1,3] ; 0,71[0,21])$. Die Differenzen zwischen beiden Patientengruppen überschritten die als minimal bedeutsamen Unterschied definierten Grenzen für alle PROs.

Schlussfolgerung: Unter den Patienten mit schwerem Asthma, die derzeit nur mit hochdosiertem ICS plus zusätzlich Controller (ohne Omalizumab) behandelt werden, erfuhren die für MG-Patienten erhebliche Gesundheitsauswirkungen und hatten eine schlechtere Asthmakontrolle, höhere Symptomlast und einen schlechteren Gesundheitszustand. Das deutet auf einen bedeutsamen bislang unbefriedigten medizinischen Bedarf unter diesen Patienten hin.

Funding: GSK (201722). Abstract zuvor präsentiert zum ATS 2016, A4330.

\section{PS48}

Adherence in patients with hypertensive crisis in the emergency department

Dr. med. Manuel Wallbach; Nadine Lach; Dr. Johanna Stock;

Dr. Henrik Hiller; Eirini Mavropoulou; Prof. Dr. Gerhard A. Müller;

Prof. Dr. med. Christoph Herrmann-Lingen; Dr. Myra-Lynn Chavanon;

Hartmud Neurath; Prof. Dr. Sabine Blaschke; Elena Lowin;

Prof. Dr. Michael J. Koziolek"

1 Universitätsmedizin Göttingen

Objective: Hypertensive crisis, an inappropriate elevation in blood pressure (BP), is a common clinical manifestation that requires immediate treatment. Estimates of the incidence of hypertensive crisis vary from $1 \%$ of all hypertensive patients over their lifetimes to $3 \%$ of all emergency department (ED) admissions. The aim of this study was to analyze adherence of patients with hypertensive crisis and to identify potential interfering co-medication or nutritive factors leading to BP elevation.

Methods: In a cross-sectional study 100 patients with hypertensive crisis (systolic BP>180 $\mathrm{mmHg}$ and/or diastolic BP>120 $\mathrm{mmHg}$ ), admitted to the ED of the University Medical Center Göttingen, were included into the study. Treatment adherence and potential interaction of co-medication and foods with antihypertensive medication that could be causal for BP elevation were analyzed by a systematic toxicological urine analysis using Gas Chromatography-Mass Spectrometry (GC-MS).

Results: From a total of 100 patients 86 were pharmacologically treated with $2.2 \pm 1.5$ antihypertensives. Due to the fact that emergency care medication was similar with regular antihypertensive regime in several patients and urine collection was performed more than $30 \mathrm{mi}-$ nutes after admission to the ED as well as limitations of the method, adherence analysis could not be performed in 24 patients. 26 patients $(42 \%)$ were designated to be adherent to antihypertensive medication. In 15 of 62 evaluated patients (24\%) complete nonadherence and in $21(34 \%)$ partial-nonadherence was found. Compared to adherent patients, nonadherent patients had a longer history of hypertension (10 vs. 15 years, $p=0.04$ ), took more antihypertensives ( $1.5 \pm 0.7$ vs. $2.8 \pm 1.1, p<0.01)$ and suffer from more comorbidities than adherent patients, but their medication schedule did not differ in the number of fixed-dose combinations. Of note, in 38 patients $(38 \%)$ non-steroidal inflammatory drugs (NSAIDs) and in 10 patients (10\%) antidepressants could be ascertained.

Conclusion: Though there are several reports using questionnaires assuming insufficient adherence, this is the first study providing adherence data from direct drug dosage analysis demonstrating the impact of nonadherence to antihypertensive medication in hypertensive crisis. Moreover, ingestion of co-medication or nutritive factors with the potential of BP elevation may additionally play part in pathogenesis. In order to prevent hypertensive crises and concomitant end-organ damage the present findings emphasize the importance for clinicians to pay more attention to the issue of adherence and co-medication.

\section{PS49}

Kasuistik: Anämie und Leukopenie einer jungen Multiple Sklerose Patientin

Dr. Laura Katharina Schenk'; Prof. Dr. Philipp Kümpers

${ }^{1}$ Universitätsklinik Münster

In unserer Notfallaufnahme stellte sich eine 22-jährige Patientin mit zunehmender Abgeschlagenheit und einem in ambulanten Kontrollen aufgefallenen Bizytopenie mit Leukopenie Hb-Abfall vor. Der behandelnde Hausarzt hatte anbetrachts dieser Konstellation einen V.a. 
Hämophagozytosesyndrom geäußert. Die Patientin berichtete über einen ca. 5 Wochen zuvor durchgemachten Atemwegsinfekt, der mit Cefpodoxim behandelt worden sei, und von welchem sie sich nicht erholt habe. Die Patient litt unter einer Multiplen Sklerose vom schubförmigen Verlaufstyp und befand sich seit zwei Jahren unter Therapie mit Fingolimod. In der klinischen Untersuchung präsentierte sich eine schlanke Patientin in stabilem Allgemeinzustand, der körperliche Untersuchungsbefund war ohne wegweisenden Befund, die Vitalparameter unauffällig. Fieber bestand nicht. Sonographisch ergab sich kein Anhalt für eine Serositis. Im Blutbild fielen Anämie $\mathrm{Hb}$ 8,6g/dl und Leukopenie 2,63Tsd/ $\mu$ l sowie im Differentialblutbildut eine absoluter Lymphopenie und ein Retikulozytenanteil von 11,2\% auf. Serumchemisch zeigte sich im Standardlabor eine leichte Hyperbilirubinämie mit vorrangige Erhöhung des indirekten Bilirubins i.S. und ein Haptoglobin $<5 \mathrm{mg} / \mathrm{dl}$, Ferritin i.S. war normwertig. In der Blutgruppenbestimmung zeigte sich ein positiver Coombs-Test. Unter dem Verdacht auf eine Autoimmunhämolytische Anämie wurde nach Versand von Material für Serologie und Erythrozytentestungen eine Steroidtherapie diskutiert. Nach Erhalt externer Vorbefunde stellte sich heraus, dass der $\mathrm{Hb}$ vom Minimum 7,19/dl kurz nach dem Infekt bereits wieder ansteigend war, sodass auf eine Therapie verzichtet wurde. Die serologischen Befunde zeigten Mycoplasma pneumoniaea IgG-AK pos. bei neg. IgM-AK. In Zusammenschau der erhobenen Befunde und des klinischen Verlaufs gehen wir von einer Coombs- positiven Hämolyse nach durchgemachter Mycoplasmeninfektion ausgehen. Differentialdiagnostisch wäre noch eine Medikamentenreaktion auf Cefpodoxim in Betracht zu ziehen, was bei Nachweis von anti-Mycoplasmen IgG jedoch unwahrscheinlich schien. Eine Coombs positive Hämolyse unter Fingolimodtherapie ist in der Literatur bisher nicht beschrieben. Hinweise auf ein Hämophyagozytosesyndrom ergaben sich bei normwertigem Ferritin, normaler Körpertemperatur und fehlender Serositis nicht, die Lymphopenie ist a.e. im Rahmen der Fingolimodtherapie zu werten. In den folgenden Blutbildkontrollen kam es zu einer Normalisierung des $\mathrm{Hb}$.

\section{PS50}

\section{Protonenpumpeninhibitoren erhöhen die Inzidenz einer spontan bakterieller Peritonitis bei Patienten mit dekompensierter Leberzirrhose und Aszites}

Tammo Lambert Tergast; Hans Laser; Prof. Dr. Michael Peter Manns; Prof. Dr. Markus Cornberg; Dr. Benjamin Maasoumy

Medizinische Hochschule Hannover

Hintergrund und Ziele: Die spontan bakterielle Peritonitis (SBP) ist eine häufige Komplikation bei Patienten mit dekompensierter Leberzirrhose und mit einer hohen Morbidität und Mortalität assoziiert. Protonenpumpeninhibitoren (PPIs) finden breite Verwendung bei Patienten mit Leberzirrhose. PPIs können durch die Erhöhung des $\mathrm{pH}$-Werts im Magen zu einer Veränderung im intestinalen Mikrobiom führen. Zurzeit ist der Einfluss von PPIs auf Inzidenz und Verlauf von SBPs Gegenstand kontroverser Diskussionen. Ziel dieser Studie war es, die Auswirkungen von PPIs auf Inzidenz und den kurzfristigen klinischen Verlauf einer SBP zu untersuchen.

Patienten und Methoden: 443 Patienten mit Leberzirrhose und Aszites, die eine Parazentese zwischen April 2013 und Juni 2016 an der Medizinischen Hochschule Hannover erhielten, wurden in diese Studie eingeschlossen. Alle Patienten wurden sorgfältig auf regelmäßige PPI-Einnahme geprüft. Sieben Patienten wurden ausgeschlossen, da bei diesen die PPI Nutzung nicht eindeutig ersichtlich war. Die verbleibenden 436 Patienten wurden für 28 Tage weiter verfolgt. Eine SBP, die mehr als 48 Stunden nach Hospitalisierung auftrat, wurde als nosokomiale SBP (nSBP) definiert.

Ergebnisse: Fast die Hälfte der Studienpopulation (48\%, $n=211 / 436)$ entwickelte entweder eine nSBP oder hatte bereits innerhalb der ers- ten 48 Stunden bzw. vor Krankenhausaufnahm erste Anzeichen einer SBP (ambulant erworbene SBP; aeSBP: $24 \%, n=50 / 211$ und nSBP: $76 \%$; $n=161 / 211)$. Patienten mit nSBP hatten eine höhere 28-Tages-Mortalität $(20 \%)$, verglichen mit den Patienten mit aeSBP $(9 \% ; p=0,05)$ und Patienten mit Aszites ohne SBP (11\%; $p=0,008)$. 77\% der Studienpopulation gaben eine regelmäßige Einnahme von PPIs an $(n=336 / 436)$. Während die Inzidenz einer SBP in der PPI Gruppe signifikant höher war als in der Gruppe ohne PPI ( $51 \%$ vs. $39 \%$; $p=0,04$; nSBP: $45 \%$ vs. $32 \%$; $\mathrm{p}=0,04$ ), war die 28-Tages-Mortalität nicht unterschiedlich (PPI 12\%, kein PPI 15\%; $p=0,38$ ). Der klinische Verlauf der SBP, in Bezug auf die Komplikationen akutes Nierenversagen und Mortalität, wurde durch eine PPI-Einnahme nicht beeinflusst (AKI: 70,2\% vs. 63,0\%; $p=0,25$ : Mortalität: $16,4 \%$ vs. $23,0 \% p=0,36$ ). Es gab keinen signifikanten Unterschied in Bezug auf die Inzidenz von Gram-positiven Bakterien zwischen den beiden Gruppen. Bei Patienten mit PPI Nutzung wurde allerdings eine numerisch höhere Inzidenz von Enterokokken-Spezies dokumentiert. Das Ansprechen auf eine empirische antibiotische Therapie blieb hiervon unbeeinflusst.

Schlussfolgerung: Die Einnahme von PPls erhöht das Risiko für die Entwicklung einer SBP bei Patienten mit Leberzirrhose und Aszites. PPIs sind aber nicht mit einer höheren 28-Tages- Mortalität assoziiert und haben keinen wesentlichen Einfluss auf den klinischen Verlauf einer SBP. Bei Patienten mit Leberzirrhose sollte die Gabe von PPIs genau geprüft werden.

\section{PS51 \\ Produktprobleme bei Companion diagnostics - Welche Tests sind betroffen? \\ PD Dr. med. habil. Rüdiger Siekmeier'; Dipl.-Bio. Jürgen Hannig'; Prof. Dr. med. Winfried März ${ }^{2}$ \\ ${ }^{1}$ Pharmazeutisches Institut der Universität Bonn; ${ }^{2}$ synlab Akademie Mannheim}

Einleitung: Seit über 15 Jahren regelt die Europäische Direktive 98/79/ EC zu In-vitro Diagnostika (IVD) Inverkehrbringen und Marktüberwachung von IVD. Companion Diagnostics (CD; Tests zur Zulassung und individuellen Anwendung von Arzneimitteln) sind von zunehmender Bedeutung, unterliegen aber in der derzeit in Überarbeitung befindlichen "alten" Direktive keinen besonderen regulatorischen Anforderungen. Diese Studie untersucht vom BfArM bei Produktproblemen für CD auf dessen Homepage (http://www.bfarm.de/DE/Medizinprodukte/riskinfo/kundeninfo/functions/kundeninfo-node.html) veröffentlichte korrektive Maßnahmen (Field safety corrective actions, FSCA) und Kundeninformationen (Field safety notes FSN).

Material und Methoden: Analysiert wurden 2005-2015 veröffentlichte FSCA und FSN. Die Definition erfolgte entsprechend einer Liste zugelassener CD der FDA (http://www.fda.gov/MedicalDevices/ProductsandMedicalProcedures/InVitroDiagnostics/ucm301431.htm).

Ergebnisse: Es fanden sich insgesamt 11 FSCA für 9 auf molekularbiologischen und 2 auf immunologischen Methoden beruhende CD. Nur 5 Assays fanden sich auf der Liste der von der FDA zugelassenen CD. Betroffen waren ausschließlich CD für Arzneimittel der Onkologie, namentlich zur Diagnostik von K-RAS (5 Fälle), Herceptin (4 Fälle), EGFR (1 Fall) und BRAF (1 Fall). In allen Fällen erfolgte ein Rückruf. Weitere Informationen wurden von den Herstellern durch die bei Rückrufen obligaten Kundeninformation zur Verfügung gestellt. Zusammenfassung: Trotz der geringen Fallzahl sind CD von großer Bedeutung, da auf deren Ergebnissen die individuelle Therapieentscheidung mit einzelnen Onkologika beruht, deren Einsatz mit erheblichen und ökonomisch relevanten Kosten einhergeht. Betroffen sind meist "ältere“, in breiter Anwendung stehende Marker, während "neuere“ Marker, da sie erst mit Zulassung der jeweiligen Arzneimittel eingeführt werden, seltener betroffen sind. Aufgrund unterschiedlicher Zulassungsver- 
fahren für IVD ("Approval" der FDA, "New approach" in Europa) und z. T. unterschiedlicher zugelassener Arzneimittel in den USA und Europa bestehen deutliche Unterschiede der auf dem Markt befindlichen Assays. Die derzeitige Neufassung der Europäischen IVD Direktive trägt der hohen Bedeutung von CD mit einer im Vergleich zur "alten“ Direktive 98/79/EC stringenteren Regulation (z. B. künftige Einbindung „Benannter Stellen“) vor erstmaligem Inverkehrbringen) Rechnung.

\section{PS52 \\ Optimierung der individuellen Medikation mittels Genotypisierung und Interaktionsprüfung Matthias Herforth \\ HMG Systems Engineering GmbH}

Im Praxisalltag sind Ärzte häufig mit Polypharmazie-Patienten konfrontiert, die schwer einstellbar sind, starke unerwünschte Arzneimittelwirkungen aufweisen oder auf die Behandlung nicht ansprechen. Die alleinige Prüfung auf Arzneimittelinteraktionen kann zwar das Risiko schwerer unerwünschter Arzneimittelwirkungen senken, aber Beschwerden oder Therapieversager, die auf die genetischen Merkmale des Patienten zurückzuführen sind, bleiben dabei unberücksichtigt. Es werden die ersten Ergebnisse eines Pilotprojektes vorgestellt, bei dem niedergelassene Ärzte personalisierte Informationen über mögliche Interaktionen zwischen Arznei-, Nahrungs- und Genussmitteln, als auch genetisch bedingte Auswirkungen auf die Metabolisierung von Arzneimitteln für den individuellen Patienten zur Verfügung gestellt bekommen. Das Projekt richtet sich hauptsächlich an schwer einstellbare multimorbide Patienten, die täglich mehr als fünf verschiedene Medikamente einnehmen. Es kommt die PGXperts Plattform zum Einsatz, die Anamneseinformationen des Patienten sowie die Genotypisierungsergebnisse für ausgewählte SNPs (Single Nucleotide Poylmorphisms) der eingeschickten Blutprobe automatisch auswertet und außerdem mögliche Wechselwirkungen zwischen allen derzeitig eingenommenen Arznei-, Nahrungs- und Genussmitteln untersucht. Als präventive Maßnahme werden auch Medikamente aufgeführt, die für potentielle zukünftige Erkrankungen aufgrund der genetischen Merkmale des Patienten klinische Konsequenzen haben könnten, prädiktive Aussagen über Erkrankungsrisiken werden nicht getroffen. Alle Ergebnisse werden in dem leicht verständlichen PerforM-Bericht (Personalisierte Information zur Medikation) zusammengefasst und erlauben dem Arzt die individuelle Pharmakotherapie zu optimieren ohne seine Therapiehoheit einzuschränken. Es wurde ein kompletter Service konzipiert, der problemlos in den Routineablauf des Arztes integriert werden kann. Der Arzt hat die Möglichkeit die Anamnese über die integrierte Webschnittstelle zu erfassen. Die anschließende Genotypisierung erfolgt mit einem maßgeschneiderten Array zur Analyse von bis zu 128 ausgewählten SNPs pro Patient. Die Genotypisierungsergebnisse werden basierend auf Algorithmen in Phänotypen übersetzt und mit bekannten Effekten auf die Pharmakokinetik des eingenommenen Medikaments als auch mögliche klinische Konsequenzen korreliert. Hierfür wurde eine pharmakogenetische Literaturdatenbank nach standardisierten Kriterien geschaffen. Darüber hinaus werden alle bekannten Interaktionen auf Basis der ABDA ${ }^{-}$-Datenbank ermittelt. Das Projekt wird wissenschaftlich vom Institut für experimentelle und klinische Pharmakologie des UKSH, Kiel und der Wilhelm Löhe Hochschule, Fürth begleitet.

\section{Präventivmedizin}

PS53

Duale Plättchenhemmung mit Clopidogrel, Prasugrel und Ticagrelor bei Patienten mit akutem Koronarsyndrom im klinischen Alltag

Safoura Sheikh Rezaei; Georg Heinze; Angelika Geroldinger; Berthold Reichardt; Michael Wolzt

Hintergrund: Die antithrombotische Therapie spielt eine zentrale Rolle bei Patienten mit akutem Koronarsyndrom (ACS). Die optimale Behandlungsdauer mit $\mathrm{P}_{2} \mathrm{Y}_{12}$ Inhibitoren wird in der Literatur hinsichtlich der Prävention eines Events, definiert durch Re-ACS oder Todesfall, kontrovers diskutiert. Das Ziel dieser epidemiologischen Studie war daher den therapeutische Nutzen sowie die Ereignisrate im klinischen Alltag bei Patienten mit einer ACS-Diagnose unter einer dualen Therapie mit Acetysalicylsäure und Clopidogrel, Prasugrel oder Ticagrelor zu untersuchen.

Methode: Für die statistischen Auswertungen wurden 72.676 Patientendaten mit einer ACS-Erstentlassungsdiagnose, codiert durch ICD-system, aus den 13 österreichischen Sozialversicherungsträgern in dem Zeitraum von 2009 bis 2014 analysiert.

Resultate: 32.830 Patientendaten mit einer Erstverordnung für einen $\mathrm{P}_{2} \mathrm{Y}_{12}$ Inhibitor innerhalb der ersten 30 Tagen nach dem Index-ACS wurden analysiert. Die Anzahl der Patienten, die mit Clopidogrel, Prasugrel und Ticagrelor behandelt wurden, betrug 18.640 (5,8\%), 6.683 $(20,4 \%)$ und 7.507 (22,9\%). Die Überlebensanalyse innerhalb eines medianen Beobachtungszeitraumes von 24,9 Monaten ergab 4.975 Events. Die kumulative Inzidenz nach 2 Jahren für Re-ACS oder Tod betrug $18,7 \%$ bei Patienten mit Clopidogrel, $8,7 \%$ bei Patienten mit Prasugrel und $12,0 \%$ bei Patienten mit Ticagrelor.

Conclusio: Die mittlere Anwendungsdauer der P2Y12-Inhibitoren bei ACS Patienten entspricht den europäschen Richtlinien. Prasugrel und Ticagrelor wurden nach Markteinführung vermehrt eingesetzt und zeigten im Vergleich zu Clopidogrel niedrigere Ereignisrate von Re-ACS- oder Todesfällen. Clopidogrel wird überwiegend bei älteren Patienten mit mehr Komorbitäten verschrieben. Für die Prävention von Re-ACS oder Todesfall in Patienten mit einem ACS empfiehlt es sich daher eine Behandlungsdauer mit einem $\mathrm{P}_{2} \mathrm{Y}_{12}$ Inhibitor von 12 Monaten.

\section{PS54}

Einfluss von Depression auf die physische Aktivität bei Patienten mit systemischem Lupus erythematodes

L. Lipovskiy; Prof. Dr. C. G. O. Baerwald'; PD Dr. O. Siefert ${ }^{1}$

${ }^{1}$ Universitätsklinikum Leipzig

Einleitung: Muskuloskelettale Beschwerden, eine verminderte Lebensqualität sowie psychische Beeinträchtigung sind charakteristisch für einen systemischen Lupus erythematodes (SLE). Regelmäßige Bewegung und physische Aktivität ist ein empfohlener Therapiebestandteil bei Patienten mit chronischen rheumatischen Erkrankungen.

Ziel: Untersuchung der Einfluss von körperlichen und psychischen Beschwerden auf die physische Aktivität bei Patienten mit SLE. Methoden Untersucht wurden 85 SLE-Patienten, $92 \%$ weiblich, Alter 24 bis 69 Jahre, mittlerer SLEDAI Score 4,95 $\pm 2,8$. Für die Erfassung der gesamten physischen Aktivität anhand der Patientenangaben wurde ein Fragebogen (International Physical Activity Questionnaire, IPAQLF) eingesetzt, daraus das metabolische Äquivalent (MET)-Minuten pro Woche (Min/Wo) berechnet. Die Einschätzung des psychischen Status erfolgte mittels des Beck-Depressionsinventars (BDI-II), des Sta- 
te-Trait-Angstinventars (STAI) und des Perceived Stress Questionnaire (PSQ). Zusätzlich wurden Schmerzscores (VAS, PainDETECT), Fragebogen zur Lebensqualität (short-form health survey, SF-36; Health Assesment Questionarie, HAQ-DI) und Gründe den Sport nicht zu betreiben erhoben. Ergebnisse Laut der WHO-Empfehlungen zu körperlicher Aktivität für gesunde Erwachsene waren 10,6 \% der SLE-Patienten physisch inaktiv $(525,2 \pm 277,3$ MET-Min/Wo), 31,8 \% hatten eine moderate körperliche Aktivität (2448 \pm 932,4 MET-Min/Woche), weitere 57,6 $\%$ Patienten waren körperlich aktiv (9906,5 \pm 4702,5 MET-Min/Woche). Körperliche Inaktivität war mit einer stärkeren Depressivität assoziiert (OR 4,5; 95\% Cl:1.2 - 17.2, p=0,04). Patienten mit moderater und schwerer Depressivität hatten auch höhere aktuelle (STAI-S, $p<0,01$ ) und habituelle (STAI-T, $p<0,01$ ) Angst sowie eine subjektive Wahrnehmung von Stress (PSQ, $p<0,03$ ) im Vergleich zu Patienten mit geringer ausgeprägter Depressivität. Darüber hinaus war eine höhere physische Aktivität mit erhöhter " "Vitalität" ${ }^{\prime \prime \prime}(\mathrm{p}<0,03)$ und " "körperliche Rollenfunktion "' $(p<0,05)$ des SF-36 assoziiert. Interessanterweise konnte kein statistisch signifikanter Zusammenhang mit der Krankheitsaktivität (SLEDAl) gezeigt werden. Die aktuelle Schmerzempfindung war signifikant höher in der Gruppe der inaktiven SLE-Patienten im Vergleich zu den Patienten mit höherer körperlicher Aktivität (VAS 83,3 \pm $29 \mathrm{~mm}$ vs. $24.7 \pm 16 \mathrm{~mm}, \mathrm{p}<0,01$ ). Außerdem zeigten diese Patienten eine Einschränkung in alltäglichen Funktionsbereichen ( $\mathrm{HAQ} 1,21 \pm$ $0,56$ vs. $0,47 \pm 0,16, p<0,05)$. Nach den Patientenangaben waren «Lupus-Schub» $(42,5 \%)$, «Müdigkeit» $(27,6 \%)$ und «Gelenkbeschwerden» $(15,7 \%)$ die häufigsten Ursachen für eine geringe physische Aktivität.

Schlussfolgerung: Bei vielen SLE- Patienten besteht eine mangelnde physische Aktivität, weswegen Programme zur Optimierung der physischen Aktivitäten im Alltag entwickelt und die Patienten entsprechend geschult werden sollten.

\section{PS55}

Fischers Fritze fischt frische Fische - hoffentlich! Fallvorstellung: Anaphylaktische Symptome bei Scombrotoxinvergiftung

Dr. Daniela Kampmeyer; Dr. Sebastian Wolfrum

Universitätsklinikum Schleswig-Holstein Campus Lübeck

Hintergrund: Etwa 1\% aller Patienten einer Notaufnahme werden aufgrund von allergischen/anaphylaktischen Reaktionen vorstellig. Bei Erwachsenen werden allergische Reaktionen meist durch Insektengifte, Medikamente oder seltener durch Nahrungsmittel ausgelöst. Treten anaphylaktische Symptome bei einer Gruppe von Erwachsenen ohne vorbekannte Allergien auf, so sollten Lebensmittelintoxikationen als Differentialdiagnose in Betracht gezogen werden.

Kasuistik: Im Juli 2016 stellten sich vier Patienten mit Übelkeit, breiigem Stuhlgang und Hautausschlag und Flush-Symptomatik in der Notaufnahme des UKSH Campus Lübeck vor. Drei der Betroffenen hatten in einem Restaurant Thunfischsteaks gegessen, eine Person hatte Thunfisch in einem anderen Restaurant verzehrt. Lebensmittelallergien waren bei keinem der Betroffenen bekannt. Die körperlichen Untersuchung ergab eine Urtikaria insbesondere im Oberkörperbereich. Laborchemisch ergaben sich keine Auffälligkeiten. Zwei von vier Patienten erhielten $\mathrm{H}_{1}$ - und $\mathrm{H}_{2}$-Blocker sowie Prednisolon. Es war eine rasche Besserung der Symptome zu verzeichnen. Bei den anderen beiden Patienten war es bereits zu einer regredienten Symptomatik gekommen. Alle Patienten konnten nach einer mehrstündigen Überwachung symptomfrei entlassen werden Pathophysiologie: In Thunfisch kann es - ähnlich wie bei anderen Fischen der Scombridae und Scomberesocidae Familie, z.B. Makrele, Hering und Sardelle - zu einer Anreicherung von Scombrotoxin kommen. Die genaue Zusammensetzung dieses Giftes ist unklar, jedoch besteht es zu einem Großteil aus Histamin. Dieses entsteht durch eine bakterielle Zersetzung von Histidin im Muskelgewebe der oben genannten Fische. Auch durch Erhitzen wird das angereicherte Histamin, das für das Auftreten von anaphylaktischen Symptome verantwortlich gemacht wird, nicht zerstört. Gelegentlich kommt es im Rahmen einer Scombrotoxinvergiftung zum Blutdruckabfall, im Extremfall zu einem Koronarspasmus und malignen Herzrhythmusstörungen.

Prävention und Epidemiologie: Verlässliche epidemiologische Daten sind nur unzureichend vorhanden. Häufig sind die Symptome der Vergiftung mild, sodass eine Notfallversorgung nicht notwendig ist. Zudem ist zur Diagnosestellung der Nachweis erhöhter Histaminkonzentrationen im Fisch notwendig. In der EU besteht eine Pflicht zur Bestimmung der Histaminkonzentration bei Fischen der Scombridae und Scomberesocidae Familien. Trotzdem kam es z.B. 2005 in England und Wales zu einer Häufung von Scombrotoxinvergiftungen mit 46 Betroffenen.

Schlussfolgerung: Da keine Lebensmittelallergien bei den Betroffenen bekannt waren und die Symptome bei allen gleichermaßen einsetzten, war das Vorliegen einer Typ-1-Allergie unwahrscheinlich. Weil es sich um Gäste aus zwei verschiedenen Restaurants handelte, ist eine Unterbrechung der Kühlkette vor Verteilung in die Restaurants anzunehmen. Das Gesundheitsamt wurde eingeschaltet - nachträglich konnte eine erhöhte Histaminkonzentration im Fisch nachgewiesen werden.

\section{PS56}

Überraschender Befund eines Sinus Venosus Defektes bei einer 44-jährigen Patientin

Ilona Hasper'; Dr. Gerd-Walter Keller; Dr. Herbert Lorenz

${ }^{1}$ Asklepios Südpfalzklinik Germersheim

Einleitung: In Deutschland findet man angeborene Herzfehler bei etwa $1 \%$ aller Kinder, wovon $17 \%$ Vorhofseptumdefekte ausmachen. Davon sind etwa 6 \% der Fälle Sinus-Venosus Defekte. 1, 2

Kasuistik: Eine 44-jährige, aus Nigeria stammende Frau, welche seit 8 Jahren in Deutschland lebt, wurde aufgenommen mit Herzrasen und Thoraxschmerzen, außerdem zunehmender Belastungsdyspnoe und Palpitationen. Eine arterielle Hypertonie war mit ACE-Hemmer und einem Calciumantagonisten behandelt.

Untersuchungsbefund bei Aufnahme: Der Blutdruck bei Aufnahme betrug $145 / 80 \mathrm{~mm} \mathrm{Hg}$. Im EKG fand sich eine Sinustachycardie, HF 113/min Rechtsschenkelblock bei einem Linkslagetyp. Die Sauerstoffsättigung betrug $100 \%$. Es war ein raues $2 / 6$ Systolikum bei gespaltenem 2. Herzton zu auskultieren.

Weitere Befunde: Im Röntgenbild fiel eine Kardiomegalie auf. In der Langzeit-EKG- Registrierung wurde eine kurze Phase paroxysmalen Vorhoffflatterns aufgezeichnet. Echokardiographisch zeigte sich eine deutliche Dilatation der rechten Herzhöhlen bei guter linksventrikulärer Funktion. Der pA systolisch betrug ca $60 \mathrm{~mm} \mathrm{Hg}$. Im TEE zeigte sich nach Kontrastmittelgabe ein Übertritt auf Vorhofebene mit Links-Rechts-Shunt im Bereich des proximalen Septums in der Nähe einer Pulmonalvene. Wir stellten so die Diagnose eines Sinus-venosus Defektes.

Therapie Und Verlauf: Nach Koronarangiographie und Kardio-MRT erfolgte am Klinikum der Stadt Ludwigshafen ein minimalinvasiver Eingriff mit Verschluß eines hochsitzenden ASD von $5 \times 2,5 \mathrm{~cm}$ mittels Pericardpatch bei gleichzeitiger Korrektur der Fehlmündung zweier Lungenvenen. In der postoperativen Verlaufskontrolle war die Dilatation der rechten Herzhöhlen echokardigraphisch deutlich regredient bei Abnahme des Pulmonaldruckes. 
Schlussfolgerrung: In Deutschland werden angeborene Herzfehler meist im Rahmen der Vorsorgeuntersuchungen entdeckt, teils sogar schon pränatal. Prinzipiell besteht bei Sinus-Venosus-Defekten immer die Indikation zum interventionellen Verschluß. Eine transthorakale Echokardiogaphie im Rahmen einer kardiologischen Diagnostik ist schon richtungsweisend. Zur genaueren Differenzierung sollte jedoch bei Rechtsherzbelastung ein TEE mit Kontrastmittelgabe erfolgen, besonders zur Diagnose eines Sinus venosus Defektes. 3 Auch bei Patienten im höheren Lebensalter mit Rechtsherzbelastungszeichen muß an ein angeborenes Vitium gedacht werde, vor allem bei nicht in Deutschland geborenen und aufgewachsenen Menschen. Erwachsene mit Sinus-Venosus-Defekt profitieren in jedem Lebensalter von einem Verschluß des Defektes.1

Quellen: 1. ESC Guidelines for the management of grown-up congenital heart disease (new version 2010) 2. Zeus T, Nickenig G. Persistierendes Foramen ovale/Vorfofseptumdefekt: Diagnostik und Therapie, Dtsch Med Wochenschr 2012;137: 194-197 3. Martin S, Shapiro E, Mukherjee M. Atrial Septal Defects - Clinical Manifestations, Echo Assessment, and Intervention. Clin.Med. Insights Cardiol. 2014; 8(Suppl1); 93-98

\section{PS57}

Walnüsse als Teil der täglichen Ernährung senken Blutlipide, unabhängig davon, ob sie als Snack oder Hauptmalzeit konsumiert werden

Charlotte Bamberger'; Andreas Rossmeier'; Dr. Katharina Lechner'; Dr. Liya Wu'; Dr. Elisa Waldmann'; Dr. Renée Stark²; Julia Altenhofer'; Kerstin Henze'; Prof. Dr. med. Klaus G Parhofer ${ }^{1}$

${ }^{1}$ Klinikum der Universität München, Campus Großhadern; ${ }^{2}$ Helmholtz Zentrum München, Deutsches Forschungszentrum für Gesundheit und Umwelt (GmbH)

Hintergrund: Aktuelle Studien zeigen einen Zusammenhang zwischen regelmäßigem Walnusskonsum und einer Verbesserung des Lipidprofils. Da noch ungeklärt ist, ob der Zeitpunkt des Nussverzehrs ausschlaggebend und ob dies auf einen gleichzeitigen Austausch von Makronährstoffen zurückzuführen ist, wurde der lipidsenkende Effekt von Walnüssen auf Nüchternlipide auf Basis einer isokalorischen Ernährung untersucht.

Methoden: In einer randomisierten, kontrollierten, prospektiven Cross-Over-Studie wurden 194 gesunde Männer und postmenopausale Frauen > 50 Jahre (134 Frauen und 60 Männer, 63 \pm 7 Jahre, BMI $25.1 \pm 4.0 \mathrm{~kg} / \mathrm{m}^{2}$ ) eingeschlossen. Nach einer 4-wöchigen nussfreien Periode wurden die Probanden 2 Interventionsgruppen zugeteilt. Die erste Gruppe ( $\mathrm{N}=96)$ erhielt zunächst über 8 Wochen eine Walnuss-angereicherte Ernährung (43 $\mathrm{g}$ Walnüsse/d) und dann nach einer 4-wöchigen Auswaschphase eine nussfreie Kontrollernährung Bei der anderen Gruppe $(n=98)$ erfolgte zunächst die Kontrolldiät und dann die Walnussdiät. Zu Beginn der Walnussphase wurden die Probanden randomisiert, in welcher Form sie die Walnüsse verzehren sollten, ob als Teil der Hauptmalzeiten ( $\mathrm{N}=99)$ oder als Zwischenmalzeit (Snack) ( $\mathrm{N}=95)$. Zusätzlich erfolgte eine Einteilung dahingehend wie sie den Energiegehalt der Walnüsse (ca. $300 \mathrm{kcal}$ ) einsparen sollten. Eine Gruppe sollte Kohlenhydrate $(\mathrm{N}=62)$, eine zweite Fett $(\mathrm{N}=65)$ und eine dritte beides $(\mathrm{N}=67)$ einsparen. Untersucht wurden Gesamtcholesterin (TC), LDL-Cholesterin (LDL-C), HDL-Cholesterin (HDL-C), non-HDL-Cholesterin (non-HDL-C), Triglyzeride (TG), ApolipoproteinB (ApoB) und Lipoprotein(a) (Lp(a)).

Ergebnisse: Verglichen mit der Kontrolle konnte unter Walnussverzehr eine signifikante Reduktion des TC (Walnuss vs Kontrolle (mg/ $\mathrm{dl}):-8,1 \pm 24,1$ vs $-1,0 \pm 22,6 ; p=0,0025)$, non-HDL-C $(-9,1 \pm 20,7$ vs $-1,3 \pm 19,2$; $p=<0,0001)$, LDL-C $(-7,2 \pm 20,3$ vs $-1,8 \pm 17,9 ; p=0,0035)$, ApoB $(-6,6 \pm 14,0$ vs $-0,6 \pm 12,5 ; p=<0,0001)$ und der TG $(-4,5 \pm 31,3$ vs $+4,0 \pm 42,8 ; p=0,0162)$ beobachtet werden. Ob die Probanden die Walnüsse als Zwischenmalzeit oder zu den Hauptmalzeiten verzehrten, zeigte keinen signifikanten Unterschied. Auch die Art der kalorischen Einsparung in den einzelnen Interventionsgruppen wies keine signifikanten Abweichungen auf.

Schlussfolgerung: Ein täglicher Verzehr von $43 \mathrm{~g}$ Walnüssen über 8 Wochen führte zu einer signifikanten Reduktion von TC, non-HDL-C, LDL-C und ApoB. Zu welchem Zeitpunkt die Walnüsse verzehrt wurden beeinflusste diesen Effekt nicht.

\section{PS58}

Akute Hepatitis-E-Infektion als Ursache einer cholestatischen Hepatitis bei einem Colitis-ulcerosa-Patienten unter Remicade-Therapie

Prof. Dr. Peter Konturek; Prof. Dr. med. Igor Harsch;

Dr. med. Laura Minnigerode; Dr. med. Stephan Heymann;

Dr. med. Thomas Hess; Dr. med. Karl-Heinz Berghäuser

Thüringen Klinik Saalfeld, Akad. Lehrkrankenhaus Univ. Jena

Einleitung: Hepatitis E ist eine virale Lebererkrankung, die durch das Hepatitis-E-Virus verursacht wird. Während in den sogenannten Endemie-Gebieten in Südostasien der Genotyp 1 und 2 typisch sind, treten in Europa sporadische Fälle des Genotyps 3 und 4 auf. Die Infektion wird in erster Linie durch den Verzehr von unzureichend gegartem Fleisch übertragen. Bei immunsupprimierten Patienten kann eine akute Hepatitis $E$ in eine chronische Infektion übergehen. Im folgenden Fall berichten wir über eine 5ojährige Patientin mit Pancolitis ulcerosa (ED: 2011). Auf Grund eines schweren akuten Schubs im Juni 2016 wurde bei der Patientin die Therapie mit einem Anti-TNF-?-Inhibitor (Remicade) eingeleitet. Unter dieser Therapie, die in der Dosis 5 mg pro kg alle 4 Wochen intravenös appliziert wurde, kam es rasch zur klinischen Besserung und zu einem signifikanten Rückgang des Calprotectin-Wertes. 5 Monate nach Beginn der Therapie wurde die Patientin mit Zeichen einer schweren cholestatischen Hepatitis in unserer Klinik stationär aufgenommen. Als Ursache wurde eine Hepatitis-E-Infektion (Genotyp 3 F) diagnostiziert. Nach der Pausierung der Anti-TNF-?-Therapie kam es spontan, innerhalb von 5 Wochen zur Normalisierung der erhöhten Transaminasen. Es bestand kein Anhalt für eine Chronifizierung der Infektion, so dass eine antivirale Therapie mit Ribavirin nicht indiziert war.

Schlussfolgerung: Bei Patienten unter Remicade-Therapie mit cholestatischer Hepatitis muss differentialdiagnostisch an die Möglichkeit einer Hepatitis-E-Infektion gedacht werden.

\section{PS59}

The CRP value at the time of implantation of permanent hemodialysis catheter as risk factor for the development of a catheter-related complication

Fani Delistefani; Dr. Manuel Wallbach; Prof. Dr. Gerhard Anton Müller; Prof. Dr. Michael J. Koziolek

Background: The prevalence of permanent hemodialysis catheters as hemodialysis access among patients with end-stage renal disease is increasing. Related complications, such as infection, have unfavorable effects on morbidity, mortality and costs. Therefore, identification of risk factors leading to those complications is required.

Aims and objective: The aim of this study was to investigate whether elevated C-reactive protein (CRP) value at the time of permanent hemodialysis catheter implantation is predictive for the development of a catheter-related complication and especially infection. 
Methods: This retrospective study examined 151 permanent hemodialysis catheters implanted in 130 patients between January 2004 and December 2013. The following data were collected at the time of catheter implantation: CRP value, history of catheter related infection, microbiological results, immunosuppression and diabetes mellitus. The next primary outcomes were recorded over the 3 months following the implantation: catheter-related infection (local or systemic), days of hospital stay and/or death. Catheter removal or revision, hospitalization and use of antibiotics were as secondary outcomes identified.

Results: Median value of CRP at the time of implantation was 24.7 $\mathrm{mg} / \mathrm{l}$ (IQR 9.4-38.3 mg/l) (normal value range 0-5 mg/l). We identified a total of $29(19.3 \%)$ infections, $23(15.3 \%)$ of them were systemic and $6(4.0 \%)$ local. The development of an infection was independent of the CRP value $(p=0.45)$ at the time of implantation as well as the presence of diabetes mellitus $(p=0.23)$ or immunosuppression $(p=0.75)$. Univariate analysis revealed that infection was more frequent in patients with previous history of germ carriage $(p<0.001)$ and in patients with history of catheter related infection $(p<0.05)$. Catheter removal or revision $(p<0.001)$, hospitalization $(p<0.001)$ and use of antibiotics $(p<0.001)$ were also more often observed in patients with previous history of germ carriage. Hospital stay was extended in patients without history of catheter-related infections [8 (IQR 4-14) vs. 3.5 (IQR 2-6) days, $\mathrm{p}<0.001]$. However, $70.2 \%$ of these patients had an acute kidney injury, so that the hemodialysis treatment had to be first initiated.

Conclusions: Our study demonstrated that the individual history of germ carriage and catheter-related infections, but not the CRP value at the time of dialysis catheter implantation take influence on the development of catheter related complication and especially infection within 3 months after implantation.

\section{PS60 \\ HDL Efflux Kapazität und kardiovaskuläre Ereignisse bei Menschen mit chronischer Nierenerkrankung Lucie Bauer'; Sabine Kern²; Kyrill S. Rogacev Rogacev³ Insa E. Emrich?; Adam Zawada?; Prof. Dr. med. Danilo Fliser?; Akos Heinemann²; Gunnar H. Heine?; Gunther Marsche ${ }^{2}$ \\ ${ }^{1}$ Klinik für Innere Medizin IV; ${ }^{2}$ Medizinische Universität Graz; ${ }^{3}$ Universitäts- klinikum Schleswig-Holstein; ${ }^{4}$ Universitätsklinikum des Saarlandes}

Fragestellung: Bei chronisch nierenkranken Menschen besteht im Gegensatz zur Allgemeinbevölkerung kein Zusammenhang zwischen hohen HDL-Cholesterin (HDL-C) Plasmaspiegeln und einem verringerten Auftreten von kardiovaskulären Ereignissen. Eine Erklärung hierfür ist, dass Urämietoxine zu einer veränderten Funktionalität des HDL-C mit verminderter Cholesterin Efflux Kapazität (CEC) führen. In rezenten Querschnittsanalysen in der Allgemeinbevölkerung und bei zumeist nierengesunden kardiovaskulären Risikopatienten konnte gezeigt werden, dass eine enge Korrelation zwischen CEC und kardiovaskulären Ereignissen besteht, die unabhängig vom $\mathrm{HDL}-\mathrm{C}$ ist. In der CARE FOR HOMe Kohorte soll nun überprüft werden, inwieweit sich der CEC als Prädiktor für kardiovaskuläre Ereignisse auch bei chronischen nierenkranken Patienten eignet.

Methodik: In der prospektiven CARE FOR HOMe Studie wurden 525 Patienten im Stadium der chronischen Nierenerkrankung $\mathrm{G}_{2}-\mathrm{G}_{4}$ (estimated glomerular filtration rate [eGFR] $15-89 \mathrm{ml} / \mathrm{min} / 1.73 \mathrm{~m}^{2}$ ) untersucht. Der primäre Endpunkt setzte sich aus nicht-fatalen atherosklerotischen Ereignissen und Tod jedweder Genese zusammen (CVE-D). Als sekundärer Endpunkt wurden nicht-fatale und fatale kardiovaskuläre Ereignisse unter Ausschluss nicht-kardiovaskulären Todesfälle gewertet (CVE). CEC wurde mittels Aufnahmekapazität apoB-armen Serums von radioaktiv markiertem Cholesterin aus J774 Makrophagen bestimmt.
Ergebnisse: In der durchgeführten Korrelationsanalyse war CEC signifikant mit HDL-C $(r=0,497 \mathrm{p}<0,001)$ und Apoliporotein A-I $(r=$ $0,563 p<0,001)$ assoziiert. CEC war bei Frauen $(12,6 \pm 2,3 \%)$ höher als bei Männer $(12,0 \pm 2,4 \% ; p=0,002)$ und niedriger bei Patienten mit Diabetes mellitus $(11,9 \pm 2,4 \%)$ als bei Patienten ohne $(12,5 \pm 2,3 \%$; $p$ $=0,011)$, unterschied sich jedoch nicht bei Patienten mit $(12,0 \pm 2,5 \%)$ oder ohne $(12,4 \pm 2,3 \% ; p=0,122)$ kardiovaskuläre Vorerkrankung bei Studieneinschluss. 142 Patienten erlitten in einer Follow-up Zeit von 4,6 \pm 2,0 Jahre den primären Endpunkt; 114 Patienten erlitten den sekundären Endpunkt. Die Patienten mit Ereignis waren älter, hatten häufiger Diabetes mellitus und eine niedrigere eGFR. Die CEC-Quartile waren weder in der durchgeführte Kaplan-Meier Analyse (log-rank $p=0,267$ [CVE-D]; $p=0,100$ [CVE]) noch in der multivariaten Co-Regressionsanalyse ein Prädiktor für kardiovaskuläre Ereignisse. HDL-C war in einer univariaten Analyse mit kardiovaskulären Ereignissen assoziiert, in einer multivariaten Analyse jedoch nicht.

Diskussion: Überraschenderweise ist der CEC kein prognostischer Marker für kardiovaskuläre Ereignisse bei Patienten mit chronischer Nierenerkrankung. Unsere Ergebnisse wurden in einer nachfolgenden Analyse der 4D Kohorte bestätigt. Zukünftig sollte untersucht werden, ob andere antiinflammatorische oder vasoprotektive Eigenschaften des HDL-C mit kardiovaskulären Ereignissen assoziiert sind.

\section{PS61 \\ Der Flüchtling als Patient eine besondere Herausforderung in Diagnostik und Therape spezieller Erkrankungsbilder, am Beispiel der Tuberkulose}

Dr. Annette Kurrle

Agaplesion Ev. Diakoniekrankenhaus Ingelheim

Anamnese: Erste Vorstellung der 26-jährigen aus Somalia stammenden Patientin mit diffusen Abdominalbeschwerden. Diagnostik: Sono, CT Abdomen abdominelle und iliakale Lymphknotenvergrößerungen. Laparoskopische Abklärung mit Exzision von Lymphknoten ergab den Befund einer unspezifischen Lymphadenopathie unklarer Ätiologie. Stationäre Aufnahme vier Monate später mit diffusen progredienten Abdominalbeschwerden, zunehmender AZ-Verschlechterung, Fieber, Gewichtsverlust von $2 \mathrm{~kg}$ in drei Monaten, zunehmend Nachtschweiß. Eigenanamnese auf Grund der sprachlichen Barrieren nicht suffizient möglich. Fremdanamnestische Angaben wurden mit Hilfe von Übersetzern erhoben, soweit eruierbar keine weiteren Vorerkrankungen.

Körperliche Untersuchung: Patientin in deutlich red. AZ und EZ, RR 120/8ommHg, Hf:131/min ,AF: $14 / \mathrm{min}$,Temperatur rectal $38,5^{\circ} \mathrm{C}$. Cardiopulmonaler Status regelrecht, Haut: trocken, Schleimhäute blass, keine dermatologischen Auffälligkeiten. Lymphknotenstatus: periphere Lymphknoten nicht vergrößert palpabel. Abdomen: gespannt, Aszites, keine Resistenzen , diffuser abdomineller Druckschmerz, Darmgeräusche regelrecht. Neurologischer Status unauffällig. Labor: Leukozyten 10700, mikrozytäre, hypochrome Anämie ( $\mathrm{Hb}$ 9,8g/dl ), Thrombozyten 549000, Ca: 2,ommol/l, CRP 147 mg/l (<0,5), LDH 267 U/I, schwere Hypoalbuminämie, $(28,6)$, weitere Eiweißfraktionen in der Elektrophorese deutlich erhöht. g-Gt und Ap leicht erhöht. ANA, ANCA im Normbereich, Quantiferontest negativ. Blutkulturen: steril

Weitere Untersuchungen: Sono Abdomen: Aszites, Splenomegalie, V.a. Lymphknotenkonglomerate iliakal. Pleurasono: kein Pleuraerguss. Echo: unauffällige Klappen, kein Perikarderguss. MRT Abdomen:Zeichen knotiger Peritonitis mit disseminierender peritonealer, mesenterialer Ausdehnung mit granulomatösen Entzündungszeichen. Vergleich zur Voruntersuchung vor 4 Monaten: deutliche Befundverschlechterung. Rö-Thorax: unauffällig keine Hilusvergrößerung, keine Rundherde. Aszitespunktion: kein Erreger 
Verlauf und Therapie: Bei rezidivierenden Fieberschüben Antibiose mit Ceftriaxon $2 \mathrm{~g}$. Nach MRT-Befund mit peritonealen Veränderungen, Befundprogredienz, der klinischen Symptomatik mit zunehmender AZ-Verschlechterung, Verlegung der Patientin in infektiologische Abteilung bei V.a extrapulmonale Tuberkulose, V.a. Peritonelatuberkulose. Dort erweiterte Diagnostik, unauffällige Kulturen für Mykobakterium, erneute Probelaparotomie Diagnose einer Peritonealtuberkulose gesichert werden.

Schlussfolgerung: Peritonealtuberkulose ist eine seltene extrapulmonale Tuberkulosemanifestation mit einer hohen Inzidenz insbesondere in Afrika, die im Rahmen von HIV-Erkrankungen, aber auch als eigenständiges Erkrankungsbild auftritt. Diagnoseerhebung gestaltet sich bei unspezifischem Symptomkomplex aus Abdominalbeschwerden mit Aszitesbildung, unspezifischen Laborveränderungen schwierig, insbesondere bei neg. kulturellem Erregerbefund. Erst mit erneuter Probelaparatomie war Sicherung der Diagnose möglich.

\section{PS62}

\section{Interleukin-13-Knockout reduziert die humorale}

Gallensäurekonzentration und Leberschädigung in Abcb4-Knockout Mäusen

Nora Helmrich; Luisa Hahn; Dr. Diran Herebian;

Prof. Dr. med. Ertan Mayatepek; Dr. Yuri Churin; Annette Tschuschner; PD Dr. Martin Roderfeld; Prof. Dr. med. Elke Roeb

'Schwerpunkt Gastroenterologie, Zentrum für Innere Medizin, Justus-Liebig Universität Gießen ${ }^{2}$ Department of General Pediatrics, Neonatology and Pediatric Cardiology, Medical Faculty, Heinrich-Heine-University, Düsseldorf, Germany

Einleitung: In Zellkultur und Tierversuchen wurde gezeigt, dass IL-13 die biliäre Epithelzell- Barriere-Funktion schwächt. IL-13 ist als Th2 spezifisches Zytokin an der hepatischen Fibrogenese beteiligt. Mit dem vorliegenden Projekt soll der potentielle therapeutische Nutzen einer IL-13-Depletion im Abcb4-Knockout Maus Modell analysiert werden.

Methoden: Durch Kreuzen von Abcb4-/- und IL-13-/- Mäusen wurden Doppel-Knockout-Hybride, auf dem Fibrose-suszeptiblen Stamm BALB/C gezüchtet. Weibliche Tiere in den Altersstufen 8,26 und 52 Wochen wurden untersucht $(n=10)$. Die Leberintegrität wurde mittels histologischer- und serologischer Tests analysiert. Auf molekularer und zellbiologischer Ebene wurden Serum und Lebergewebe mit Hilfe von Western Blot, Gallensäureanalyse, Hydroxyprolinwertbestimmung, Immunhistochemie und qRT-PCR untersucht.

Ergebnisse: Wir haben im Abcb4-Knockout Maus Modell für Cholestase nachgewiesen, dass ein Ausschalten von IL-13 eine ca. 12-fache Verringerung der Gallensäurekonzentration im Serum zur Folge hat Darüber hinaus wurde eine Verbesserung der Leberintegrität (ALT) eine verminderte hepatozytäre Apoptose, und im Langzeitverlauf eine verminderte Fibrose beobachtet. Gallengangsarchitektur und Zell-Zell-Kontakte sind in Abcb4-/-/IL-13-/- Doppel-Knockout-Mäusen deutlich weniger gestört als in Abcb4-/-Mäusen.

Diskussion: Ein IL-13 knockout verbessert die Barrierefunktion von Gallengangsepithelien, mit konsekutiv niedrigeren humoralen Gallensäurekonzentrationen von Abcb4-/-/IL-13-/- Mäusen. Die vorliegende Studie erweitert das molekularbiologische Verständnis des durch IL-13 beeinflußten Gallensäuremetabolismus und der damit verbundenen hepatischen Cholestase. Die Modulation des IL-13-Signalweges könnte einen neuen therapeutischen Ansatz für cholestatische Erkrankungen darstellen.

\section{PS63}

Lebensbedrohliche Sinusvenenthrombose als seltene Komplikation eines M. Crohn bei einem 20-jährigen Patienten

Ruth Mair'; Cagla Nur Demirci; Vlad Pavel; Franz-Xaver Dechant;

Prof. Dr. Martina Müller; Dr. Stephan Schmid

' Uniklinikum Regensburg

Hintergrund: M. Crohn zählt zu den chronisch entzündlichen Darmerkrankungen (CED) und betrifft v.a. jüngere Patienten. Bekannt sind sowohl intestinale als auch extraintestinale Manifestationen. In Zusammenhang mit CED wird auch eine Hyperkoagulabilität beschrieben. Unter den thromboembolischen Komplikationen treten v.a. tiefe Beinvenenthrombosen und Lungenembolien auf. Das Auftreten einer Sinusvenenthrombose (SVT) ist selten. Fall Ein 20-jähriger Patient stellte sich mit seit 14 Tagen bestehenden holocephalen Kopfschmerzen vor, die sich in den letzten $24 \mathrm{~h}$ aggravierten und im Stehen stärker waren. 2 Jahre zuvor wurde ein M. Crohn diagnostiziert. Die Behandlung erfolgte mit Salofalk und steroidhaltingen Klistieren. Ein manifester Eisenmangel war bekannt.

Diagnostik: Klinisch und neurologisch zeigten sich bis auf einen endgradigen Nackenbeugeschmerz keine Auffälligkeiten. Die Vitalparameter waren unauffällig. Laborchemisch zeigte sich lediglich ein leicht erhöhter D-Dimer Wert. Die durchgeführte MR-Bildgebung des Schädels ergab die Diagnose einer SVT des linken S. sigmoideus sowie des rechten S. transversus. In den weiterführenden Untersuchungen ergab sich kein eindeutiger H.a. eine cerebrale Entzündung. Das Thrombophiliescreening war unauffällig. Von Seiten des M. Crohn ergab sich endoskopisch eine floride Entzündung.

Verlauf: Umgehend erfolgte unter intensivmedizinischer Überwachung eine Antikoagulation mittels Heparin, worunter sich die Symptomatik rasch besserte. Nach Umstellung auf eine orale Antikoagulation trat eine $\mathrm{Hb}$-wirksame untere Gl-Blutung auf, die gut beherrscht werden konnte. Unter Therapie zeigte sich die floride Entzündung im kurzfristigen Verlauf rückläufig. Weitere Blutungen traten nicht mehr auf. Im Verlauf klagte der Patient nicht mehr über Kopfschmerzen. Neurologische Symptome zeigten sich nicht. In der Kontrollbildgebung war der Thrombus kaum mehr nachweisbar. Der Patient befindet sich in hervorragendem Zustand und kann sein Studium mittlerweile uneingeschränkt fortsetzen.

Schlussfolgerungen: CED ist ein unabhängiger Risikofaktor für das Auftreten von Thromboembolien. Grund dafür ist u.a. eine Hyperkoagulabilität. Thromboembolien treten v.a. während akuter Krankheitsschübe auf. Bei hospitalisierten Patienten wird deshalb eine Thromboseprophylaxe, auch bei blutigen Diarrhoen empfohlen. Andere Risikofaktoren sollten minimiert werden. Bei Notwendigkeit einer längerfristigen Antikoagulation sollte die remissionserhaltende Therapie intensiviert werden, um ein zusätzliches Blutungsrisiko zu vermindern. Die SVT ist eine seltene, aber schwerwiegende thromboembolische Komplikation bei CED. Es sind eher junge männliche Patienten betroffen. Häufigstes Symptom sind Kopfschmerzen. Eisenmangel scheint ein Risikofaktor zu sein. Bei dieser Symptomkonstellation ist es wichtig, an die SVT zu denken, da es sich um eine gefährliche Komplikation handelt. Unter adäquater Therapie ist jedoch ein hervorragendes Outcome zu erzielen wie der obige Fall zeigt. 


\section{Schnittstelle Kardiologie / Adipositas / Diabetes 1}

\section{PS64}

T1 and T2 Mapping for Evaluation of Myocardial Involvement in Patients with ANCA-associated Vasculitides

Dr. Simon Greulich'; Dr. Agnes Mayr; Daniel Kitterer; Jörg Latus; Jörg Henes; Niko Braun; Philipp Kaesemann; Alexandru Patrascu; Prof. Dr. med. Mark Dominik Alscher; Prof. Dr. med. Udo Sechtem; Heiko Mahrholdt

${ }^{1}$ Robert-Bosch-Krankenhaus

Background: Myocardial involvement in AAV patients might be silent, presenting with no or nonspecific symptoms, normal ECG, and preserved left-ventricular ejection fraction (LV-EF). Since up to $50 \%$ of deaths in these patients may be due to myocardial involvement, a reliable diagnostic tool is warranted. In contrast to LGE-CMR, which has its strengths in detecting focal inflammatory or fibrotic processes, recent mapping techniques are able to detect even subtle, diffuse inflammatory or fibrotic processes. Our study sought to investigate ANCA (antineutrophil cytoplasmic antibody)-associated vasculitides (AAV) patients for myocardial involvement by a cardiovascular magnetic resonance (CMR) protocol, including late gadolinium enhancement (LGE) and mapping sequences.

Methods: Thirty-seven AAV patients were prospectively enrolled and underwent CMR imaging. Twenty healthy volunteers served as controls.

Results: Mean LV-EF was 64\%; LGE prevalence of the AAV patients was $43 \%$. AAV patients had higher median native $\mathrm{T}_{1}$ (988 vs. $952 \mathrm{~ms}$, $\mathrm{p}<0.001$ ), lower post-contrast $\mathrm{T}_{1}$ (488 vs. $524 \mathrm{~ms}, \mathrm{p}=0.03$ ), expanded extracellular volume (ECV) ( 27.5 vs. $24.5 \%, \mathrm{p}<0.001)$, and higher $\mathrm{T} 2$ ( 53 vs. $49 \mathrm{~ms}, \mathrm{p}<0.001$ ) compared to controls, with most parameters independent of the LGE status. Native $T_{1}$ and $T_{2}$ in AAV patients showed the highest prevalence of abnormally increased values beyond the $95 \%$ percentile of controls. Conclusion: AAV patients demonstrated increased $T_{1}, E C V$, and $T_{2}$ values, with native $T_{1}$ and $T_{2}$ showing the highest prevalence of values beyond the $95 \%$ percentile of normal. Since these findings seem to be independent of LGE, mapping techniques may provide complementary information to LGE-CMR in the assessment of myocardial involvement in patients with AAV.

\section{PS65 \\ Einfluss von Adipokinen auf die Differenzierung der Spongiosa-abgeleiteten mesenchymalen Stammzellen von Arthrosepatienten}

Lali Tsiklauri'; Janina Werner; Dr. Klaus Frommer; Rosel Engel;

Prof. Dr. med. Stefan Rehart; Sabine Wenisch;

Prof. Dr. med. Ulf Müller-Ladner; Prof. Dr. Elena Neumann

${ }^{1}$ Justus-Liebig-Universität Gießen; Kerckhoff-Klinik GmbH

Der altersbedingte Knochenverlust korreliert mit einer erhöhten Fettinfiltration im Knochenmark. Daher findet sich das osteoporotisch veränderte Hartgewebe direkt an der Grenze zu den Adipozyten des Knochenmarks. Das Fettgewebe ist metabolisch hochaktiv. Es sezerniert unter anderem Adipokine (zB Visfatin, Resistin und Leptin), die möglicherweise die Differenzierung im Knochenmark lokalisierten mesenchymalen Stammzellen (MSC) beeinflussen und zum fortschreitenden Knochenverlust an den Knochen-Knochenmark-Grenzflächen in Rahmen der Osteoporose (OP) beitragen können. Daher wurde die Expression der Adipokine im Knochen osteoporotischer und nicht-osteoporotischer Spender mit Arthrose sowie ihre Effekte auf die osteogene und adipogene Differenzierung von MSC untersucht. Nach der Isolierung von RNA aus Spongiosa humaner Hüftköpfe sowie aus differenzierten MSC wurden Genexpressionsanalysen mittels Real-Time PCR durchgeführt. Die Differenzierung von MSC erfolgte über 21 Tagen mit bzw. ohne Zugabe von Adipokinen (Visfatin, Resistin, Leptin). Die Sekretion von Entzündungsmediatoren wurde mittels ELISA gemessen. Alizarin Red S Färbung diente zum Nachweis von mineralisierter Matrix. Für Spongiosa-Transfer Experimente wurde die Spongiosa unter sterilen Bedingungen aufgereinigt (zellfrei) und die MSC zur Differenzierung in die Spongiosa gegeben. Das Expressionslevel von Visfatin und Leptin war signifikant im osteoporotischen Knochen ( $n=14)$ erhöht, während Resistin deutlich herunterreguliert war. Visfatin führte zu einer signifikanten Erhöhung von IL-6, IL-8 und MCP-1 während der adipogenen sowie osteogenen Differenzierung. Eine verstärkte Mineralisierung durch Visfatin konnte in Rahmen der Osteogenese nachgewiesen werden, wobei die Kollagen Typ 1 Expression durch Visfatin signifikant herabgesetzt war (zB Tag 21: 4,6-fach). Im Gegensatz zu Resistin und Leptin bewirkte Visfatin eine signifikante Reduktion von $\mathrm{MMP}_{13}$ ( $\mathrm{zB}$ Tag 21: 3,2-fach), MMP2 (zB Tag 21: 2,4-fach), TIMP1 (zB Tag 21: 3,2-fach), TIMP2 (zB Tag 21: 4,3-fach) und RunX2 (zB Tag 21: 5,8-fach) in osteogen differenzierten Zellen. Während der adipogenen Differenzierung führte Visfatin zu einem signifikanten Expressionsanstieg von MMP13 (zB Tag 21: 104 \pm 88$)$. Zwar war die Visfatin-induzierte MMP13-Expression während der Adipogenese auf gereinigten autologen Spongiosa-Fragmenten nachweisbar, dieser Effekt fiel aber deutlich geringer aus als unter Standard-Zellkulturbedingungen. Die Ergebnisse weisen darauf hin, dass Visfatin die Differenzierung der MSC beeinflusst: Visfatin verstärkt die Mineralisierung, reduziert aber die Expression von Kollagen Typ 1. Dies könnte zu einem erhöhten Fragilitätsrisiko führen. Durch die Reduktion der RunX2-Expression verringert Visfatin das osteogene Differenzierungspotential. Die Visfatin-induzierte Dysregulation der MMP- und TIMP-Expression sowie von Entzündungsmediatoren trägt möglicherweise zu einem gestörten Knochenumbau an den Knochen-Knochenmark-Grenzflächen bei der OP bei.

\section{PS66}

A rare cause for alveolar hemorrhage and acute kidney failure

Dr. Milenka Cabrera; Dr. Christian Löffler; Priv.-Doz Urs Benck;

Prof. Dr. Bernhard Krämer

Universitätsmedizin Mannheim

We present a case of a 42-year-old female with no medical history who was admitted to our department with hemoptysis, fever, rapidly progressive renal failure, lower limb edema and severe hypertension $(240 / 100 \mathrm{mmHg}$ ). These symptoms had been occurring progressively within the last 7 days prior to admission. Urinalysis revealed microhematuria and a nephrotic-range proteinuria. Chest radiographs and computed tomography showed bilateral infiltration suggestive of alveolar hemorrhage. The kidneys presented with hyperechogenic parenchyma and were of normal size in renal sonography. Bronchoscopy with bronchoalveolar lavage confirmed alveolar hemorrhage and ruled out an infection. Steroid pulse therapy, plasmapheresis and cyclophosphamide were started because of a strong suspicion of immune mediated pulmonary renal syndrome as seen in ANCA associated vasculitis or Goodpasture's syndrome. Due to severe fluid overload and refractory hypertension despite antihypertensive treatment hemodialysis was initiated. Renal biopsy findings showed no evidence of active necrotizing glomerulonephritis or crescent formation, however, a severe lumen constricting arteriosclerosis, severe thrombotic microangiopathy and mild tubular damage. Testing for proteinase 3 and myeloperoxidase specific ANCA (PR3-/MPO-ANCA), antinuclear autoantibodies (ANA), anti-GBM antibodies and anti-phospholipid antibodies were negative. C-reactive protein levels and erythrocyte sedimentation rate were not suggestive of an inflammatory disease. We therefore considered malignant hypertension to be the underlying diagnosis in this case. Pathophysiologically, hypertensive vascular stress can cause acute endothelial damage leading to alveolar hemorrhage and renal failure. To date, only very few cases are published regarding this rare manifestation (pulmonary alveolar 
hemorrhage) of malignant hypertension. Plasmapheresis and immunosuppressive treatment were discontinued immediately. In the further course pulmonary hemorrhage completely dissolved under treatment with antihypertensive agents. The renal function, however, did not recover. Four months after discharge the patient continued to be dialysis dependent, her blood pressure was normal and chest radiographs showed no pathological findings. Although the clinical characteristics, course and outcome of malignant hypertension are well defined, we present an unusual manifestation with pulmonary alveolar hemorrhage and acute kidney failure mimicking an immune mediated pulmonary renal syndrome. Once the diagnosis of diffuse alveolar hemorrhage is established malignant hypertension should be taken into account as a differential diagnosis in the proper clinical setting.

\section{PS67 \\ Evaluation of Myocardial Fibrosis in Non-ischemic Dilated Cardiomyopathy and its Prognostic Value Dr. Elham Kayvanpour'; Dr. Farbod Sedaghat-Hamedani; Daniel Li; Firat Tugrul; Prof. Niels Grabe; Bernd Lahrmann; Lutz Frankenstein; Christian Stock; Esther Herpel; Sebastian Buss; Prof. Dr. Hugo A. Katus; Benjamin Meder \\ ${ }^{1}$ Medizinische Universitätsklinik Heidelberg}

Introduction: Myocardial fibrosis and extracellular matrix expansion occur during pathological remodeling of the heart and is associated with worse outcome of affected patients. On a molecular level, novel circulating biomarkers aim to assess cardiac fibrotic remodeling. Imaging tools, such as cardiac MRI implement Gadolinium contrast agent or T1 mapping to detect larger fibrotic formations. More sufficiently, LV endomyocardial biopsies (LV-EMB) are used to assess cardiac fibrosis, to identify the individual causes (e.g. viral replication), and to aid differential diagnosis. In this study we evaluated the prognostic values of the mentioned three methods in a large DCM cohort with a follow up period of up to 10 years.

Methods: We included a total number of 542 patients who had undergone left ventricular EMB (LV-EMB) due to suspected DCM. Left ventricular collagen volume fraction (LV-CVF) was quantified using automated image processing of high-resolution scans of LV-EMB. 13 major circulating fibrosis biomarkers were measured using Human Magnetic Luminex Screening Assays. The extent of LGE was estimated as a percentage of LV-mass using the software IntelliSpace Portal with application of the "“Full-Width-Half-Maximum" (FWHM) protocol. Univariate as well as multivariate analyses between histopathological and clinical findings of patients were carried out. End-points were all-cause mortality and heart failure associated events: a composite of cardiovascular death, cardiopulmonary resuscitation, heart transplantation, sustained ventricular tachycardia, and adequate ICD treatment. ROC analyses were performed to evaluate the accuracy of different circulating fibrosis biomarkers in outcome prediction compared to LV-CVF.

Results: Using machine learning techniques, we established an automated method for quantification of LV-CVF in patients' LV-EMBs. We could show that LV-EMB is a safe procedure with a total complication rate of 2.3 in 100 patients in our center with no case of procedure-related death. Kaplan Meier survival curves showed that the extent of LV-CVF is an independent predictor of all-cause mortality $(p=0.009)$ and heart failure associated events $(p<0.0001)$. In a subgroup of 150 patients with available CMRI at time point of LV-EMB taking, no significant correlation could be observed between the absence and presence of mid-wall fibrosis or the extent of LGE. ROC analyses showed that histopathological evaluation of LV-CVF offers higher specificity and sensitivity in outcome prediction compared to circulating fibrosis biomarkers.
Conclusions: Our results suggest that $\mathrm{EMB}$ is a reliable and precise tool to characterize DCM tissue properties and strengthen the role of LV-EMB in evaluation of clinical course and outcome of patients with cardiomyopathy.

Key words: dilated cardiomyopathy, pathological remodeling, left ventricular endomyocardial biopsy

\section{PS68}

Improvement of Impaired Diastolic Left Ventricular Function after Diet-induced Weight Reduction in Severe Obesity

Sevda Karimian; Prof. Dr. med. Jürgen Stein; Dr. Boris Bauer;

Dr. med. Claudius Teupe

DGD Clinics Sachsenhausen

Background/Objectives: Obesity is independently associated with left ventricular diastolic dysfunction and altered cardiac morphology. Morbidity and mortality in patients with diastolic dysfunction are similar to values observed in patients with systolic heart failure. We hypothesised that dysfunctional cardiac response in obese individuals is reversible after weight loss. Thus, we studied the effect of dietary weight reduction on left ventricular diastolic function as well as on cardiac structure, using transthoracic echocardiography and tissue Doppler imaging.

Subjects/Methods: Thirty-two subjects with obesity underwent a 12-week low calorie fasting phase of a formula diet (OPTIFAST ${ }^{\oplus}{ }^{52}$ ). Echocardiographic tissue Doppler indices of diastolic function and measurements of cardiac size were obtained prior to and after the fasting phase. Results: A 12-week diet significantly reduced body mass index, from $40.3 \pm 6.6$ to $33.2 \pm 6.1 \mathrm{~kg} / \mathrm{m}^{2}(p<0.01)$. Weight loss was associated with a significant reduction in blood pressure and heart rate. Echocardiography revealed diastolic dysfunction in subjects with obesity, which was improved by dieting. After weight loss, transmitral Doppler echocardiography showed a significant reduction in A-wave velocity, from $65.8 \pm 19.2 \mathrm{~cm} / \mathrm{s}$ to $57.0 \pm 16.8 \mathrm{~cm} / \mathrm{s}$, and an increase in E/A-ratio, from $1.2 \pm 0.4$ to $1.4 \pm 0.5$ ( $p<0.01)$. Tissue Doppler imaging displayed a significantly lower a'-wave velocity $(10.3 \pm 2.3 \mathrm{~cm} / \mathrm{s}$ and 8.9 $\pm 1.7 \mathrm{~cm} / \mathrm{s} ; \mathrm{p}<0.01)$. Left atrial and ventricular dimensions were normal and remained unchanged after dieting.

Conclusion: Obesity is associated with diastolic dysfunction. A 12week low calorie diet with successful weight loss can reduce blood pressure and heart rate and partially normalise diastolic dysfunction.

\section{PS69}

TOPP-ABI - Tissue Optical Perfusion Pressure-Ankle Brachial Index: A new and improved method for diagnosis of PAOD Prof. Dr. med. Georg Horstick'; Laura Messner ${ }^{2}$; Dr. med. Bruno Betsch; Dr. med. Christian Georg ${ }^{3}$; Prof. Dr. med. Christine Espinola-Klein? ${ }^{1}$ Praxis für Gefäß- und Herz-Medizin, Neustadt a.d. Weinstrasse; ${ }^{2}$ Universitätsmedizin der Johannes Gutenberg-Universität - Zentrum für Kardiologie - Kardiologie I; ${ }^{3}$ Radiologie Neustadt; ${ }^{4}$ Universitätsmedizin der Johannes Gutenberg-Universität Mainz - Zentrum für Kardiologie - Kardiologie I

Einleitung: The routine diagnostic for classification of PAOD is the measurement of the $\mathrm{ABI}$ either by doppler pressure or automated oscillometric determination (AOD). AOD shortens time of analysis and reduces measuring errors. In the past interventions of the foot arteries increased with improvement of the techniques and therefore it is of utmost interest to measure tissue perfusion pressure at the level of the toe. Performing TBI (toe brachial index) a second examination after the routine $A B I$ measurement is needed. However, TBI is not always credible and toe pressure measurement (TPM) would afford a third examination inducing ischemia reperfusion pathology affecting 
the results.. Therefore, we added optical infrared sensors on the first toe of each foot measuring the pulsation of the arteries in the tissue for determination of TOPP simultaneous to the AOD of the ABI.

Methods: AngE system was used to determine ABI with AOD between ranges of 160 to $40 \mathrm{mmHg}$. TOPP at the first toe was documented simultaneously in $10 \mathrm{mmHg}$ increments while deflating the pressure cuffs. To compare TOPP versus the Doppler signal and TPM 20 healthy people were examined with the method of optical pulsation (TOPP) detected at the measuring point of the Doppler probe at the ADP and compared to the TOPP and TPM at the toe. For clinical examination 100 patients with PAOD Fontaine class Ila, Ilb and III were examined by TOPP-ABI at rest and after defined walking exercise, extensive ultrasound examination including ankle peak systolic velocity (APSV) and either MRA or CTA. 20 patients w/wo PAOD and normal TOPP-ABI and ultrasound examination without MRA or CTA served as controls. Additionally, puls wave analysis was performed to measure puls wave index (PWI) and central puls wave velocity (CPWV).

Results: TOPP in healthy subjects is 30 to $60 \mathrm{mmHg}$ higher versus TMP. The first optical pulsation at the measuring point of the Doppler pressure at the ADP correlates with the TOPP at the toe. There was no correlation to the PWV. TOPP correlates to the free of pain walking distance and the severity of PAOD in the MRA/CTA. TOPP presented a very good correlation to the APSV, $\mathrm{ABI}$ and PWI.

Conclusion: TOPP-ABI improves automated oszillometric $A B I$ determination by measurement of the systolic tissue optical perfusion pressure at the far end of microperfusion at the toe simultaneously in one examination. This standardized technique prevents human measurement errors and ischemia reperfusion effects by multiple measurements and provides new insights into foot artery hemodynamics on top of routine $A B I$ measurements.

\section{PS70 \\ Kardiomyopathie mit reduzierter EF als Risikofaktor für die Entstehung einer Sekundär sklerosierenden Cholangitis - Präsentation von 3 Fällen \\ Dr. Felix Müller \\ Klinikum Fulda gAG}

Einleitung: Die sekundär sklerosierende Cholangitis bei kritisch kranken Patienten (secondary sclerosing cholangitis in critically ill patients (SSC-CIP)) ist charakterisiert durch eine progrediente Cholestase und führt mit unterschiedlicher Latenz zu einer biliären Zirrhose und Leberversagen. Ischämische Läsionen der Gallenwege, z.B. im Rahmen eines prolongierten hämodynamischen Schocks, stellen eine plausible Erklärung der Entstehung dar. Da nur ein kleiner Teil dieser Patienten eine sekundär sklerosierende Cholangitis entwickelt, scheinen weitere Trigger notwendig. Vorliegend präsentieren wir 3 Patienten mit Sekundär Sklerosierender Cholangitis mit sehr unterschiedlichem Verlauf, die eine bekannte oder neu diagnostizierte Kardiomyopathie mit reduzierter Ejektionsfraktion aufwiesen.

Fall 1: 71-jähriger Patient mit bekannter ischämischer Kardiomyopathie und Z.n. ACVB-Operation, der posttraumatisch eine fulminante Lungenembolie entwickelte.3 Monate später stellte er sich erneut mit dem Bild einer Cholangitis vor. In der Bildgebung mittels ERCP und MRCP bestand das Bild einer Sekundär sklerosierenden Cholangitis mit biliary cast.

Fall 2: Es stellte sich eine 88-jährige, mobile Patientin bei uns zur Abklärung einer cholestatischen Hepatopatie vor. Bei ischämischer Kardiomyopathie erfolgte bereits 6 Jahre zuvor eine ACVB-Operation mit Rekonstruktion der Mitralklappe. Postoperativ kam es zu wiederholt zu hypotonenen und asystolen Phasen. Im Verlauf bestanden erhöhte
Cholestasewerte bei asymptomatischem Verlauf. Bei akutem Anstieg der Werte im Rahmen einer Darm-Operation zeigte sich in der ERCP das typische Bild einer Sekundär Sklerosierenden Cholangitis.

Fall 3: Eine 47-jährige Patientin mit schwerem Asthma bronchiale wurde bei Exazerbation auf der Intensivstation intubiert. Nachfolgend invasive Beatmung sowie 1-malige Phase eines Druckabfalls mit Reanimationsbehandlung. Echokardiographisch bestand eine deutlich reduzierte Ejektionsfraktion von ca. $25 \%$ unklarer Genese. Knapp 2 Wochen nach Aufnahme entwickelte die Patientin eine Cholangiosepsis. Im Verlauf kam es zu rapide steigenden Cholestasewerten, in der ERCP bestand das Bild einer Sekundär Sklerosierenden Cholangitis mit biliary cast. Bei fulminantem Verlauf verstarb die Patientin an Komplikationen eines Leberversagens 10 Wochen nach Aufnahme.

Diskussion: Die Fälle zeigen den äußerst unterschiedlichen Verlauf einer Sekundär Sklerosierenden Cholangitis (SSC-CIP), vom langjährig asymptomatischen Verlauf bis hin zum akuten Leberversagen. Bei Patienten mit reduzierter Ejektionsfraktion besteht ein reduziertes Herzminutenvolumen/Herzindex. Bei schwerer, intensivmedizinisch therapierter Erkrankung besteht bei diesen Patienten ein erhöhtes Risiko für eine prolongierte Gewebsminderperfusion. Somit könnte eine Kardiomyopathie mit reduzierter Ejektionsfraktion einen Triggerfaktor für die Entstehung einer Sekundär Sklerosierenden Cholangitis darstellen.

\section{Schnittstelle Kardiologie / Adipositas / Diabetes 2}

\section{PS71}

Die Rolle des extrazellulären Kalziums

\section{bei Adipositas-induzierter Inflammation}

Dr. med. Stephan Thrum; Manuela Rossol; Matthias Blüher; Prof. Dr. Ulf Wagner

${ }^{1}$ Universitätsklinikum Leipzig

Adipositas und Autoimmunerkrankungen gehen mit einer Migration von Abwehrzellen sowie einer chronischen Inflammation einher, v.a. durch die Aktivierung von Makrophagen. Patienten mit Autoimmunerkrankungen, die gleichzeitig unter einer Adipositas leiden, zeigen einen schwereren Verlauf der Grunderkrankung. Extrazelluläres Kalzium (exCa2+) fungiert im Bereich der Inflammation als Warnsignal und Verstärker jener durch die Aktivierung des NLRP3 Inflammasoms. Jedoch ist die Rolle von exCa2+ bei der Adipositas-induzierten Inflammation bisher nicht untersucht worden. Daher werden hier sowohl Proben von Fettgewebe und entzündlich-rheumatisch verändertem Synoviagewebe als auch aus Monozyten differenzierte Makrophagen (MDM) von Patienten untersucht, die unter einer Adipositas und/oder rheumatoiden Arthritis (RA) leiden. Für in vitro-Untersuchungen wurde hierzu ein Modell der MDM-Reifung unter autologen Serum entwickelt, um Probenden-spezifische Makrophagen-Eigenschaften zu untersuchen. Dabei zeigt sich unter inflammatorischen Bedingungen eine vermehrte IL-1?-Sekretion der Makrophagen in Abhängigkeit von der zugegebenen Kalziumkonzentration und dem untersuchten Probanden. Eine Expression des bei Monozyten für diesen Mechanismus wesentlichen G-Protein-gekoppelten, calciumsensitiven Rezeptors (CaSR) konnte in Westernblot nachgewiesen werden, wohingegen GPRC6A eine untergeordnete Rolle spielt. Im Verlauf soll ein neu entwickeltes Kokultur-Modell von autolog gereiften MDM und menschlichen Adipozyten genutzt werden, um die Abhängigkeit der inflammatorischen Reaktion von der Zell-Zell-Interaktion im Fettgewebe zu untersuchen. Mittels einer Fettgewebskultur kann die Reaktion von Gewebsmakrophagen in einem physiologischen Umfeld untersucht werden. In Gewebeproben sollen die Konzentration des exCa2+ sowie die CaSR-Expression im Gewebe bestimmt werden, mit Fokus auf 
Areale mit Nekrose und Inflammation. Aufbauend auf prospektiv rekrutierte RA-Patienten mit oder ohne Adipositas werden wir den Einfluss von exCa2+ auf die MDM-Subpopulationen sowie Phänotyp und die Klinik der Erkrankungen analysieren. Auf diesem Weg lässt sich die in vitro vorhandene, inflammatorische Wirkung von exCa2+ auf Makrophagen auch auf Gewebe übertragen und dessen Rolle in der Pathogenese der genannten Erkrankungen prüfen. So können mögliche neue prognostische Marker aufgezeigt werden, welche in der Folge helfen, therapeutische Ansätze der Inflammation-vermittelten Komorbidität und Morbidität bei adipösen Patienten mit Autoimmunerkrankungen zu identifizieren.

\section{PS72 \\ Neprilysin als Prädiktor kardialer Ereignisse bei chronisch nierenkranken Patienten}

Kathrin Untersteller'; Linda Feuer; Dr. Insa Emrich; Prof. Jean-Marie Launay; Prof. Alexandre Mebazaa; Prof. Alain Cohen-Solal; Dr. Nicolas Vodovar; Dr. Matthieu Legrand; Prof. Dr. med. Danilo Fliser; Prof. Dr. Gunnar Heine ${ }^{1}$ Universitätsklinikum des Saarlandes

Hintergrund: Die aktuellen Leitlinien der European Society of Cardiology betrachten das Hormon Neprilysin (NEP) als zentralen Angriffspunkt in der Therapie der systolischen Herzinsuffizienz. In der Allgemeinbevölkerung konnte bei Menschen mit Herzinsuffizienz gezeigt werden, dass erhöhte Plasma NEP-Konzentrationen mit einem schlechteren ereignisfreien Überleben assoziiert waren. Es ist unklar, ob dies auch für Menschen mit chronischer Nierenerkrankung (CKD) zutrifft, welche eine besonders hohe Inzidenz kardialer Erkrankungen haben. Daher haben wir die Plasma NEP-Konzentration und die Plasma-NEP-Aktivität als Prädiktoren für das kardiale Überleben von CKD-Patienten untersucht.

Methoden: Wir haben bei 544 CKD-Patienten (geschätzte [estimated] glomeruläre Filtrationsrate [eGFR] $15-89 \mathrm{ml} / \mathrm{min} / 1,73 \mathrm{~m}^{2}$ ) aus der fortlaufenden CARE FOR HOMe Studie die Plasma-NEP-Konzentration und -Aktivität sowie BNP im Blut gemessen. Die Patienten wurden über 4,4 2,1 Jahre hinsichtlich des Auftretens zweier primärer Endpunkte nachverfolgt: (1) Kardiale Dekompensation (HF) und (2) Kardiale Dekompensation/Tod (HF/D). Wir erstellten Korrelationsanalysen und verglichen mittels Kaplan-Meier-Kurven das ereignisfreie Überleben in Abhängigkeit von NEP-Konzentration und -Aktivität. Ergebnisse: In univariabler Korrelationsanalyse waren die NEP-Konzentration und die NEP-Aktivität weder miteinander $(r=0,007)$ noch mit eGFR (eGFR und NEP-Konzentration: $\mathrm{r}=0,036$; eGFR und NEP-Aktivität: $r=0,090$ ) oder BNP (BNP und NEP-Konzentration: $r=0,010$; BNP und NEP-Aktivität: $r=-0,127)$ assoziiert. 59 Patienten erlitten während der Nachbeobachtungszeit eine kardiale Dekompensation, während 121 Patienten den zweiten primären Endpunkt HF/D erreichten. In univariabler Kaplan-Meier-Analyse waren nach Stratifizierung der CKD-Patienten in Tertile weder eine hohe NEP-Konzentration noch eine hohe NEP-Aktivität mit einer erhöhten Wahrscheinlichkeit für eine kardiale Dekompensation assoziiert; vielmehr war eine niedrige NEP-Aktivität ein signifikanter Prädiktor für den zweiten primären Endpunkt HF/D (log-rank Test: NEP-Konzentration HF/D: $p=0,563$; HF: $p=0,477$; NEP-Aktivität HF/D: $p=0,047 ; H F: p=0,093)$. Nach Adjustierung für die eGFR war die NEP-Aktivität ebenfalls nicht mehr signifikant mit dem vermehrten Auftreten von HF/D assoziiert, während BNP weiterhin ein starker unabhängiger Prädiktor für das ereignisfreie Überleben blieb.

Schlussfolgerung: Wir konnten weder eine hohe NEP-Konzentration noch eine hohe NEP-Aktivität als klare Prädiktoren für ein schlechteres Überleben bei chronisch nierenkranken Menschen identifizieren. Die positiven Resultate aus Kohorten der weitgehend nierengesunden Allgemeinbevölkerung, welche NEP als kardialen Prädiktionsmarker definierten, können daher nicht unkritisch auf CKD-Patienten übertragen werden. Somit bleibt die prognostische Implikation von NEP bei CKD-Patienten bislang unklar und bedarf weiterer Untersuchungen.

\section{PS73}

Association of established predictors of NT-proBNP varies significantly across its quantiles in older people: The ActifE Study

Dr. med. Dr. phil. Dhayana Dallmeier'; Dr. Ulrike Braisch'; Prof. Dr. Wolfgang Koenig ${ }^{2}$; Prof. Dr. Dietrich Rothenbacher ${ }^{3}$; Prof. Dr. Michael D. Denkinger ${ }^{1}$

${ }^{1}$ Agaplesion Bethesda Klinik Ulm / Geriatrisches Zentrum Ulm/Alb-Donau;

${ }^{2}$ Deutsches Herzzentrum München; ${ }^{3}$ Universität Ulm

Background: N-Terminal pro Brain Natriuretic Peptide (NT-proBNP) is released from the ventricular myocardium in the settings of volume and/or pressure overload, and is recognized as a good marker for myocardial hemodynamic stress. We evaluated the association of established predictors of NT-proBNP across its quantiles in older people.

Methods: NT-proBNP levels were measured in 1506 community-dwelling adults ?65 years, participants at the longitudinal cohort study Activity and Function in the Elderly in Ulm study - ActiFE UIm. Quantile regression evaluated the association of age, sex, body mass index (BMI), smoking, cystatin C based glomerular filtration rate (GFR), hemoglobin, testosterone, C-reactive protein (CRP), history of hypertension, diabetes, myocardial infarction, heart failure, and stroke with NT-proBNP levels measured at baseline at the $0.10,0.25,0.50,0.75$ and 0.90 quantiles.

Results: In our study population ( $n=1456$, mean age 75.5 years, $56.9 \%$ male) NT-proBNP was not normally distributed with a median level of 153.0 ng/L [interquartile range Q1 81.9, Q3 318.0]. Simple quantile regressions showed a significant association for age, sex, GFR, hemoglobin, testosterone, CRP, history of hypertension, myocardial infarction, heart failure and stroke. In the multivariable analysis we observed a steady significant increment of the $B$-estimates across the $0.10,0.25$, $0.50,0.75$, 0.90 quantiles for following variables: age ( $(2.2,3.5,5.7,11.8$, 17.9), CRP ( $(1.3,3.4,6.9,8.2,14.2)$, hypertension ( $(3.0,18.7,34.7,63.7,80.7)$, myocardial infarction ( $(49.2,96.4,213.5,290.7,684.8$ ), and heart failure ( $332.8,49.7,106.9,418.5,704.7)$. In addition increments of BMI ( $B-1.3$, $-3.0,-5.1,-5.7,-12.9)$, GFR ( $(-0.5,-1.0,-1.8,-3.9,-9.8)$ and hemoglobin ( $\beta$ $-7.7,-8.9,-19.4,-32.4,-30.1)$ were significantly associated with lower levels of NT-proBNP across the quantiles. No statistically significant association could be detected for sex, testosterone, smoking, history of diabetes and of stroke.

Conclusion: Our data showed not only that age, BMI, GFR, hemoglobin, CRP, history of hypertension, myocardial infarction and stroke are associated with the baseline levels of NT-proBNP after further adjustment for sex, smoking, testosterone, and history of diabetes, but also that their level of contribution varies considerably across the $0.10,0.25$, $0.50,0.75$ and 0.90 quantiles, with the higher $ß$-estimates observed by history of myocardial infarction, heart failure, and hypertension. These results emphasize the need to deepen our understanding about the pathophysiology of NT-proBNP, so that the assumption of a linear relationship between these established predictors and NT-proBNP in older people should be treated with the utmost caution. Next steps should analyze the effects of these predictors across quantiles of NT-proBNP changes over time, allowing the identification of those factors with the most beneficial or rather detrimental effect. 


\section{PS74}

\section{Arrhythmogenic late sodium current is substantially caused by neuronal Nav1.8 channel in the human failing heart}

Steffen Pabel'; Dr. Nataliya Dybkova; Dr. Shakil Ahmad; Dr. Nico Hartmann; Petros Tirilomis; Dr. Thomas Fischer; Prof. Dr. Norbert Frey;

Prof. Dr. Gerd Hasenfuß; Prof. Dr. med. Samuel Tobias Sossalla

${ }^{1}$ Georg-August Universität Göttingen

The late sodium current (INaL) is a persistent $\mathrm{Na}$ influx throughout the action potential, which is enhanced in heart failure (HF). INaL deteriorates altered cellular electrophysiology resulting in an increased susceptibility for arrhythmias. Although it has been shown that phosphorylation of voltage-gated sodium channels (Nav) due to CaMKII? increases INaL in HF, the detailed underlying mechanisms of an enhanced arrhythmogenic INaL remain unclear. This study aims to investigate the role of the neuronal $\mathrm{Na}$ channel NaV1.8 (Scn1oa) for INaL generation in HF. For electrophysiological and molecular experiments we studied left ventricular samples from patients with end-stage HF $(n=23)$ and healthy control myocardium $(N F, n=10)$. We could show for the first time an isoform $\mathrm{Na}$ channel switch in $\mathrm{HF}$ consisting of increased expression levels of $\mathrm{NaV}_{1.8}$ in HF compared to control. On the contrary, the predominant cardiac isoform NaV1.5 was downregulated. Interestingly, co-immunoprecipitation showed a significant increase in association of Nav1.8 with CaMKII? in HF compared to NF. In isolated human cardiomyocytes from patients with end-stage $\mathrm{HF}$, patch clamp experiments revealed that inhibition of $\mathrm{NaV}_{1} .8$ with the novel specific blockers A-803467 (30 nmol/L) and PF-01247324 (1 $\mu \mathrm{mol} / \mathrm{L}$ ) significantly decreased INaL and thereby shortened the pathologically prolonged action potential duration. The frequency of proarrhythmic diastolic calcium sparks and, most importantly, the incidence of cellular arrhythmias (delayed afterdepolarizations and spontaneous action potentials) were potently reduced in the presence of either A-803467 or PF-01247324 in human HF cardiomyocytes. To validate these pharmacological findings derived from human preparations, we additionally investigated mice lacking Nav1.8 (SCN10A-/-) vs. wild-type (WT). Interestingly, we found a decreased INaL, a slightly abbreviated action potential duration and less arrhythmias in isoproterenol-stimulated cardiomyocytes from SCN10A-/- compared to WT. Moreover, in WT NaV1.8 inhibition resulted in significantly reduced INaL, action potential duration, frequency of arrhythmogenic calcium sparks and diminished incidence of cellular arrhythmias. These effects were absent in SCN10A-/-. In conclusion, this study demonstrates for the first time (1) a differential regulation of $\mathrm{NaV}_{1.8}$ and $\mathrm{NaV}_{1} .5$ in human $\mathrm{HF}$ and its (2) proarrhythmogenic consequences, which can (3) directly be counteracted by specific compounds. Thus, inhibition of NaV1.8 constitutes a completely novel and selective therapeutic approach, which merits further investigation.

\section{PS75}

\section{Herzinsuffizienz ist mit einer Depletion des intestinalen} Mikrobioms assoziiert

Thorben Winkler'; PD Dr. Mark Lüdde'; Dr. Femke-Anouska Heinsen²; Malte C. Rühlemann ${ }^{2}$; Dr. med. Martina E. Spehlmann'; Amer Bajrovic'; Prof. Dr. Wolfgang Lieb ${ }^{3}$; Prof. Dr. Andre Franke'; Prof. Dr. Stephan J. Ott'; Prof. Dr. Norbert Frey ${ }^{1}$

${ }^{1}$ Universitätsklinikum Schleswig-Holstein, Campus Kiel; ${ }^{2}$ Institut für Klinische Molekularbiologie, Universität Kiel; ${ }^{3}$ Institut für Epidemiologie, Universität Kiel

Fragestellung: Die Mortalität der chronischen Herzinsuffizienz (HI) bleibt trotz aktueller Behandlungsansätze hoch. Neue Therapiekonzepte werden daher dringend benötigt. Jüngste Forschungsergebnisse legen einen Einfluss des intestinalen bakteriellen Mikrobioms auf die Inzidenz und den klinischen Verlauf der Herzinsuffizienz nahe. Diese Studie sollte klären, ob charakteristische Veränderungen im intestinalen Mikrobiom von HI-Patienten nachgewiesen werden können.

Methoden und Ergebnisse: Die intestinalen Mikrobiome von 20 Patientinnen und Patienten mit Herzinsuffizienz und reduzierter linksventrikulärer Ejektionsfraktion aufgrund von ischämischer oder dilatativer Kardiomyopathie wurden mittels Hochdurchsatz-Sequenzierung der bakteriellen $16 \mathrm{~S}$ rRNA Gene untersucht. Die erstellten mikrobiellen Profile wurden mit denen passender gesunder Kontrollen, die den Fällen bestmöglich in Bezug auf Alter, Geschlecht, Body Mass Index (BMI) und Nikotinkonsum entsprachen, verglichen $(n=20)$. Der Shannon-Index, ein Maß intraindividueller alpha-Diversität basierend auf der Verteilung einzelner taxonomischer Einheiten (OTUs), zeigte bei HI-Patienten eine nominell signifikant verminderte Diversität gegenüber den Kontrollen $(P$ nom. $=0.01)$. Weitere Tests auf Basis des bakteriellen Gattungsvorkommens zeigten ebenfalls eine tendenziell verminderte alpha-Diversität bei HI-Patienten. Die Analyse verschiedener beta-Diversitätsmaße (interindividuelle Diversität) erbrachte eine signifikante Separation von HI-Patienten und Kontrollen (z.B. weighted UniFrac: $\mathrm{P}=0.004)$. Dabei zeigte sich bei HI-Patienten gegenüber den Kontrollen ein signifikant vermindertes Vorkommen der intestinalen Kerngruppen Coriobacteriaceae, Erysipelotrichaceae und Ruminococcaceae auf Familienebene. Auf Gattungsebene waren Blautia und Collinsella sowie zwei Mitglieder der Familien Erysipelotrichaceae und Ruminococcaceae bei HI-Patienten signifikant erniedrigt.

Schlussfolgerungen: HI-Patienten zeigen eine signifikant verminderte Diversität des bakteriellen intestinalen Mikrobioms sowie das verminderte Vorkommen intestinaler Kerngruppen. Unsere Untersuchungen deuten auf ein verändertes intestinales Mikrobiom als möglichen Faktor in der Pathogenese und Progression der Herzinsuffizienz hin.

\section{PS76}

The true rate of drug related cough of the angiotensin converting enzyme inhibitors in placebo controlled trials in cardiovascular medicine: A meta-analysis

Davor Vukadinovic'; Dr. Daniel Lavall; Aleksandra Nikolovska Vukadinovic; Prof. Dr. Stefan Wagenpfeil; Prof. Dr. Michael Böhm

Universitätsklinikum Homburg

Background: Angiotensin converting enzyme inhibitors (ACEi) represents a cornerstone therapy for patients with arterial hypertension, after myocardial infarction and with heart failure. Physicians often stop cardiovascular medications due to presumed adverse effects(AE). In a considerable number of patients, the same adverse effects occur also on placebo(Pla). This study aimed to investigate the rate of true drug(Dr) related cough in randomized, placebo controlled clinical trials, which are cited in European Guidelines of Cardiology for arterial hypertension, coronary heart disease and heart failure.

Methods: We performed a meta-analysis including randomized, placebo controlled trials reporting cough as an adverse effect. Studies with a minimum of 100 individuals in each group (drug and placebo arm) and a minimum follow-up of one month were included. We evaluated the drug-related effects (ACEi attributable fraction), that are attributable to the use (exposure) of $A C E i$, according to the equation: True DrAE (\%) = (DrAE(\%) - PlaAE(\%)) / DrAE(\%).

Results: Cough Num. of studies Risk Ratio, Cl 95\% Drug AE Placebo AE True Drug AE ACEi 19 2.60 (2.03-3.33) 12.41 \% 7.29 \% 41\% Arterial hypertension 66.49 (4.00-10.53) $2.82 \% 0.42 \% 85 \%$ Coronary heart diseases 102.67 (1.84-3.88 12.53 \% 7 \% 44\% Chronic heart failure 31.53 (1.11-2.11) $24.38 \% 18 \% 26 \%$ 
Conclusion: In randomized trials more than half of cough events (59\%) were not related to the ACEi treatments. These rates differ between underlying conditions with highest rates of true drug-related in arterial hypertension and lowest in heart failure. Therefore, other causes of cough e.g. congestion in chronic heart failure, should be comprehensively evaluated in patients on ACEi.

\section{PS77}

Short-term cognitive function following coronary artery bypass grafting in patients at high risk of cerebrovascular events preliminary results from a prospective study

Sarah Hosang; Dr. med. Claudius Bartels; Dr. med. Michael Hansen; Mike Herr; PD Dr. med. habil. Thomas Schilling; Dr. med. Hassina Baraki; Prof. Dr. med. Ingo Kutschka; Dr. med. Johannes Hadem

Otto-von-Guericke-Universität Magdeburg

Objectives: Short-term cognitive impairment following coronary artery bypass grafting (CABG) has been attributed to older age and several other risk factors associated with cerebrovascular disease. In an ongoing prospective study, we aim to characterize perioperative alterations in cognitive function as well as the management implications of regional cerebral-tissue oxygenation $\left(\mathrm{rSO}_{2}\right)$ monitoring in patients at high risk of cerebrovascular events. Patients with symptomatic carotid stenosis and/or previous cerebral infarctions were excluded.

Methods: Clinical parameters were collected prospectively at our institution (DRKS-ID: DRKSoooo8853). Several cognitive domains were examined on the day preceding CABG and again on postoperative day 5-7, using the Montreal Cognitive Assessment (MoCA), Trail-making Test (TMT A/B), Regensburg Word Fluency Test (RWT), and the Boston Naming Test (BNT). Patients were randomly assigned to a control vs. an intervention group. The latter received intraoperative frontal $\mathrm{rSO}_{2}$ monitoring as well as appropriate measures to optimize $\mathrm{rSO}_{2}$ in case of desaturations. Results are presented as medians (interquartile ranges). Baseline and postoperative test results were compared by Wilcoxon test.

Results: Ten patients at high risk of cerebrovascular events were included. Two out of 10 patients (20\%) were female, and median age was 68 $(62-77)$ years. Four patients $(40 \%)$ received additional mitral valve surgery. Length of cardiopulmonary bypass was 135 (100-191) min. Nine of 10 patients showed chronic kidney disease (CKD), and all patients suffered from diabetes and/or CKD. Six patients $(60 \%)$ had a reduced left ventricular ejection fraction $(<50 \%)$. Sequential neuropsychological testing revealed a modest short-term cognitive decline in 2 out of 5 assessments: MoCA 21 (17-26) vs. 22 (14-24, $p=0.122)$, TMT A 37 (31-44) vs. 39 (28-47 sec, $p=0.721)$, TMT B 99 (88-117) vs. 112 (100-152 sec, $p=0.012)$, RWT 69 (59-83) vs. 63 (49-72, $p=0.066)$, BNT 29 (24-29) vs. 29 (26-30, $p=$ 0.038 ). The $\mathrm{rSO}_{2}$ area under the curve (desaturations below $50 \%$ * time [min], mean value of left and right frontal channel) in those 5 patients randomly assigned to the $\mathrm{rSO}_{2}$ monitoring group was 1 (o-18) $\mathrm{min} \%$. Patients were discharged from hospital after 9.5 (8-10) days.

Conclusion: These preliminary results suggest that patients at high risk of a cerebrovascular event experience a modest short-term cognitive impairment following cardiac surgery, but recover soon thereafter. This ongoing study aims to characterize perioperative changes in cerebrovascular disease and to clarify the potential benefits of intraoperative cerebral $\mathrm{rSO}_{2}$ monitoring.

\section{PS78}

Ausgedehnte systemisch embolisierende Staphylococcus aureus-Aortenklappenendokarditis durch peripheren Dialyseshunt

Dr. Armin Frille'; Dr. Lorenz Weidhase'; Gudrun Borte ${ }^{2}$; Prof. Dr. Andreas Hagendorff'; Prof. Dr. Dietrich Pfeiffer'; Prof. Dr. Sirak Petros ${ }^{1}$

${ }^{1}$ Universitätsklinikum Leipzig; ${ }^{2}$ Universitätsklinikum Leipzig| Klinik und Poliklinik für Diagnostische und Interventionelle Radiologie

Hintergrund: Infektionen peripherer Dialyseshunts stellen ein hohes Risiko für die Entwicklung einer Endokarditis mit septischen Embolien dar und schlagen sich in einer erhöhten Mortalität nieder.

Kasuistik: Eine 61-jährige Patientin wurde mit Fieber $\left(39,4^{\circ} \mathrm{C}\right)$, Schüttelfrost, Luftnot und Verwirrtheit vorgestellt. Bekannt waren bisher eine koronare Zwei-Gefäß-Herzerkrankung (KHK), eine arterielle Hypertonie, eine chronisch obstruktive Lungenerkrankung und eine Langzeitdialysepflichtigkeit über einen peripheren arteriovenösen (AV-) Shunt infolge einer diabetischen Nephropathie. Aktuell fielen umschriebene druckschmerzhafte, rötliche, nicht erhabene Hautveränderungen an den Handflächen beider Hände und strichartige subunguale Einblutungen auf. Laborchemisch imponierten eine Leukozytose von 19/nl und ein C-reaktives Protein von $340 \mathrm{mg} / \mathrm{l}$. Bei initialem Verdacht auf eine pneumogene Sepsis erfolgte eine kalkulierte Antibiose mit Piperacillin/Tazobactam. Mehrere Blutkulturen wurden abgenommen und aufgrund der oben ausgeführten klinischen Zeichen eine transösophageale Echokardiographie zum Ausschluss einer Endokarditis veranlasst. Blutkulturen waren mehrmals positiv für Staphylococcus aureus ohne Resistenz, sodass die antimikrobielle Therapie auf Flucloxacillin umgestellt wurde. In der transösophagealen Echokardiographie stellten sich flottierende Strukturen (17 $\mathrm{mm}$ ) an der rechts- und akoronaren Tasche der Aortenklappe im Sinne einer floriden Aortenklappenendokarditis dar. In der Ausbreitungsdiagnostik zeigte die CT multiple eingeschmolzene Läsionen pulmonal beidseits, hepatisch, lienal, rechts renal und im rechten Posteriorstromgebiet als Hinweis für zerebrale thrombembolische Infarkte. Bei bestehender KHK zeigte die aktuelle Koronarangiographie Instent-Restenosen in den Stromgebieten der Rami interventricularis anterior und circumflexus. Bei großer emboliefähiger, infektiöser Vegetation erfolgte eine komplikationslose Implantation einer biologischen Aortenklappenprothese (Carpentier-Edwards Perimount-Aortic, Gr. 21) sowie eine koronaren Bypassoperation.

Schlussfolgerung: Der Kasus veranschaulicht das eindrucksvolle Ausmaß einer systemisch gestreuten bakteriellen Aortenklappenendokarditis mit zerebraler, pulmonaler, hepatischer, lienaler, renaler und kutaner Beteiligung. Infektiöse Eintrittspforte war am ehesten der zur ambulanten Hämodialyse verwendete periphere AV-Shunt, der initial zum Befall von Lunge und Aortenklappe führte. Die Vigilanzminderung ließ sich durch die zahlreichen zerebralen Einschmelzungen erklären. Zudem imponierten Osler-Knötchen an den Händen und subunguale Splinter-Hämorrhagien. Die Akuttherapie umfasste die zielgerichtete, antimikrobielle Therapie des Erregers in ausreichender Dauer und eine Beseitigung der Erregerquelle. Bei Risikopatienten ist es nach wie vor entscheidend, an eine Endokarditis zu denken und die erforderlichen diagnostischen und therapeutischen Maßnahmen so früh wie möglich durchzuführen. 


\section{Versorgungsforschung 1}

\section{PS79}

Zwei Jahre im Leben eines COPD Patienten: Ergebnisse der nicht-interventionellen DACCORD Studie Prof. Dr. Heinrich Worth'; Prof. Dr. Roland Buhl2 ; Prof. Dr. Carl-Peter Crieé3; Dr. med. Peter Kardos?; Dr. Claudia Mailänder?; Dr. Nadine Lossi?; Prof. Dr. Claus Vogelmeier?

${ }^{1}$ Facharztforum Fürth; ${ }^{2}$ Universitätsmedizin Mainz; ${ }^{3}$ Evangelisches Krankenhaus Göttingen?Weende; ${ }^{4}$ Zentrum für Pneumologie, Allergie, Somnologie an der Klinik Maingau; ${ }^{5}$ Novartis Pharma GmbH; ${ }^{6}$ Universitätsklinikum Marburg

Einleitung: Erkenntnisse aus randomisierten kontrollierten klinischen Studien spielen eine wichtige Rolle in der Entwicklung neuer Therapieoptionen, lassen sich aber häufig nur bedingt auf die Behandlung von COPD Patienten in der täglichen Praxis übertragen. Im Rahmen der nicht-interventionellen, prospektiven DACCORD Beobachtungsstudie wurden Exazerbationen von COPD Patienten aller Schweregrade über 2 Jahre erfasst und deren COPD Schweregrad nach GOLD 2011 jährlich bestimmt.

Methoden: In die DACCORD Studie wurden COPD Patienten eingeschlossen, bei denen der Beginn oder eine Änderung der COPD Erhaltungstherapie erfolgten. Von 3137 Patienten lagen 2-Jahresdaten vor. Exazerbationen wurden in den 6 Monaten vor Einschluss und alle 3 Monate während der Teilnahme an der Studie erhoben. Für die vier COPD- Schweregrade nach GOLD 2011 wurden Exazerbationen, Lungenfunktion und Symptome (COPD Assessment Test; CAT) zu Beginn (BL), nach 1 und 2 Jahren evaluiert.

Ergebnisse: In dieser Kohorte war der Phänotyp des Nicht-Exazerbierers stabil (Exazerbationsrate 0,26 im Jahr 1 vs. 0,25 im Jahr 2) - 69,4\% der Patienten ohne Exazerbationen in den 6 Monaten vor Studieneinschluss, berichteten auch keine Exazerbation in der gesamten Nachbeobachtungszeit von 2 Jahren. Patienten mit Exazerbationsanamnese hingegen (? 1 Exazerbation vor Einschluss) wiesen eine Exazerbationsrate von 0,770 im Jahr 1 bzw. 0,663 im Jahr 2 auf. Häufig-Exazerbierer (?2 Exazerbationen in den 6 Monaten vor Studieneinschluss) hingegen zeigten mit 1,34 Exazerbationen im Jahr 1 und 0,99 Exazerbationen im Jahr 2 die höchsten Exazerbationsraten. Bei Studieneintritt wurden 44.6\% der Patienten den GOLD-Gruppe D zugeordnet, ein Drittel allein aufgrund Ihrer Exazerbationshistorie. Die Einteilung der Patienten in die COPD Schweregrade nach GOLD 2011 ist für den Verlauf der Studie in Tabelle 1 dargestellt.

Tabelle 1: COPD Schweregrad nach GOLD 2011 an: Baseline, Jahr 1, Jahr 2 (Anzahl Patienten in \%) A: 6.1\%, 8.8\%, 10.7\% B: 44.6\%, 54.6\%, 52.8\% C: $3.2 \%, 2.8 \%, 2.7 \%$ D: $46.1 \%, 31.1 \%, 32.1 \%$

Schlussfolgerungen: Exazerbationen sind in der täglichen Praxis eher selten. Der Phänotyp des nicht-exazerbierenden COPD Patienten erscheint stabil. Das Risiko eine Exazerbation zu erleiden steigt mit dem Vorliegen von Exazerbationen in der Vorgeschichte.

\section{PS80 \\ Preliminary results of a prospective multi-center registry of Lower Esophageal Sphincter Stimulation for GERD: The LESS-GERD Registry \\ Prof. Dr. med. Joachim Labenz'; Dr. Henning G. Schulz; Dr. Andreas Leodolter; Dr. Ralf Weise \\ 1 Diakonie Klinikum GmbH, Jung-Stilling-Krankenhaus}

Introduction: Safety and effectiveness of electrical stimulation of the lower esophageal sphincter (LESES) using the EndoStim ${ }^{\circledast}$ LES Stimu- lation System (Nijmegen, The Netherlands) was demonstrated in clinical trials . Limited data is available on outcomes in clinical practice.

Aims \& Methods: An ongoing, prospective international multicenter web-based registry in patients with disruptive GERD symptoms, treated with LES-ES is collecting data at baseline and at routine followups for 5 years. Demographics, adverse events, GERD symptoms, GERD health related quality of life scores (GERD-HRQL), structured GI symptom questionnaires for extraesophageal symptoms, use of proton pump inhibitors (PPIs) and physiological data (esophageal pH / manometry) are collected when available.

Results: Data was available from 147 patients at baseline enrolled at 11 sites, 87 patients with 6 months postop follow up and 50 patients with 12 months follow up. 89\% (77/87) of patients showed an improvement in their GERD-HRQL score on LES-ES at 6 month and 92\% (46/50) showed an improvement at 12 month compared to baseline. The median composite GERD-HRQL score improved from 23 preoperatively to 8 at 6 month $(p=0.007)$ and from 21 to 6 at 12 month $(p=0.03)$. At baseline, $49 \%$ of patients (29/59) complained of bothersome heartburn symptoms affecting sleep. Out of those, 79\% (23/29) where improved by month 6 and $98 \%$ (40/41) where improved by month 12 . At baseline, $48 \%$ and $20 \%$ of subjects reported moderate or severe regurgitation, respectively which decreased to $20 \%$ and $7 \%$ at 6 months $(n=54)$ and $12 \%$ and $0 \%$ at 12 months $(n=26)$. All patients were on daily prescription of PPI at baseline. At $6 \mathrm{~m}$ post op $75 \%$ (75/99) were completely off

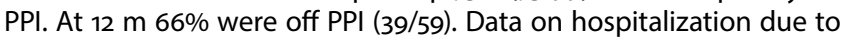
GERD was available from 107 patients at baseline and 54 patients reaching $12 \mathrm{~m}$ off therapy. Annualized hospitalization rates due to GERD pre EndoStim was 1.1 days/year and 0.13 days/year at 12 months. Safety data was adjudicated by an independent DSMB. Four serious adverse events in three patients were reported. One myocardial infarction related sudden death at 11 month postop, not related to device or procedure was reported. One event of lead erosion into the esophagus was detected during routine endoscopy. The device was removed during laparoscopic fundoplication procedure. Two events of gastroparesis, possibly related to the device requiring hospitalization were reported in one patient.

Conclusion: Electrical stimulation of the LES is safe and effective in clinical practice in treating GERD patients with disruptive GERD symptoms despite PPI. Improvement in symptom scores appears to be progressive over 12 months. LES stimulation results in significant improvement in GERD outcomes and reduced GERD-related healthcare resource burden. LES-ES should be considered a viable treatment option for treating GERD patients with disruptive GERD symptoms despite maximal medical management.

\section{PS81}

Alginat als add-on bei GERD mit unzureichendem PPI-Effekt: Die LOPA III Studie

Prof. Dr. med. Joachim Labenz'; Matthias Müller; Dr. Daniela Borkenstein Diakonie Klinikum GmbH, Jung-Stilling-Krankenhaus

Hintergrund: Die gastroösophageale Refluxkrankheit (GERD) ist sehr häufig. Protonenpumpeninhibitoren (PPI) sind die Mittel der ersten Wahl für alle Manifestationen der GERD. Nationale und internationale Studien haben aber gezeigt, dass viele Patienten trotz PPI Symptome beklagen. In vorangegangenen Studien in der hausärztlichen Versorgung konnte gezeigt werden, dass $46 \%$ der chronischen GERD-Patienten mit PPI-Dauertherapie an mindestens 2 Tagen pro Woche weiterhin Symptome aufweisen und jeder 5. Patient mit der Behandlung unzufrieden ist. Eine mögliche Ursache für Durchbruchsymptome unter PPI ist die " "Acid Pocket "“" , die erfolgreich mit einem Alginat behandelt werden kann. Es ist unbekannt, ob eine Bedarfstherapie mit einem Alginat die Patientenzufriedenheit mit der Therapie verbessert. 
Patienten und Methode: In einer prospektiven, multizentrischen Observationsstudie in hausärztlichen Praxen $(n=13)$ werden Patienten mit der Diagnose einer chronischen GERD und einer PPI-Dauertherapie seit mindestens 1 Jahr, die mit der Behandlung unzufrieden sind (Score 1 oder 2 auf einer fünfstufigen Likert-Skala) zusätzlich zu ihrer laufenden PPI-Therapie mit einem Alginat (Gaviscon dual) nach Bedarf über 14 Tage behandelt. Die Patienten dokumentieren den Alginat-Bedarf und bewerten am Ende der Therapie erneut ihre Symptome und die Zufriedenheit mit der Therapie. Primäres Studienziel ist die Beurteilung der Patientenzufriedenheit auf der fünfteiligen Likert-Skala.

Ergebnisse: In die noch laufende Studie wurden bisher 85 Patienten aufgenommen und behandelt. Es handelt sich um 37 Männer und 48 Frauen mit einem medianen Alter von 56 Jahren (Bereich 18-85 Jahre). Die Ergebnisse von 82 Patienten konnten ausgewertet werden. Insgesamt gaben 54 der 82 Patienten (66\%) eine Verbesserung ihrer Therapiezufriedenheit um mindestens 1 Punkt auf der fünfteiligen Likert-Skala an (1 Punkt: $n=24,2$ Punkte: $n=20,3$ Punkte: $n=9$, 4 Punkte: $n=1)$. Neunzehn Patienten schätzten ihre Zufriedenheit unverändert ein. Schwerwiegende unerwünschte Ereignisse wurden nicht erfasst.

Zusammenfassung: Eine " "Add-on" ${ }^{\prime \prime \prime}$-Therapie mit einem Alginat ist eine einfache, sichere und wirksame Option zur Behandlung persistierender Refluxsymptome unter PPI bei chronischen Refluxpatienten, die mit einer alleinigen PPI-Therapie unzufrieden sind.

\section{PS82}

Wirkung von Vedolizumab auf Patienten-relevante Endpunkte bei Colitis ulcerosa: Ergebnisse eines deutschlandweiten Registers Christian Langbein'; Prof. Dr. med. Raja Atreya ${ }^{2}$; Dr. Tony Bruns ${ }^{3}$; Prof. Dr. med. Axel Dignass?; Dr. Katrin Ende?; Prof. Dr. Jochen Hampe?; Prof. Dr. Franz Hartmann?; Prof. Dr. Markus F. Neurath²; Dr. Jochen Maul?; Priv.-Doz Jan C. Preiß?; Dr. Renate Schmelz?; Prof. Dr. Britta Siegmund'?; Priv.-Doz Carsten Schmidt ${ }^{3}$; Dr. Hermann Schulze?; Priv.-Doz Niels Teich"1; Prof. Ulrike von Arnim ${ }^{12}$; Prof. Dr. med. Daniel C. Baumgart ${ }^{13}$;

Prof. Dr. med. Andreas Stallmach ${ }^{3}$

${ }^{1}$ Universitätstäklinikum Jena; ${ }^{2}$ Friedrich-Alexander Universität Erlangen-Nürnberg; ${ }^{3}$ Universitätsklinikum Jena; ${ }^{4}$ Agaplesion Markus Krankenhaus; ${ }^{5}$ HELIOS Krankenhaus Erfurt; ${ }^{6}$ Universitätsklinikum Dresden; ${ }^{7}$ Gastroenterologie am Bayerischen Platz; ${ }^{8}$ Charité Medical School Berlin; ${ }^{9}$ TU Dresden, Universitätsklinikum Dresden; ${ }^{10}$ Charité - Campus Benjamin Franklin; ${ }^{11}$ Internistische Gemeinschaftspraxis für Verdauungs- und Stoffwechselkrankheiten; ${ }^{12}$ Klinikum der Otto-von-Guericke-Universität Magdeburg; ${ }^{13}$ Charité - Universitätsmedizin Berlin, Campus Virchow-Klinikum

Einleitung: Für die Beurteilung der therapeutischen Effektivität bei Patienten mit chronisch-entzündlichen Erkrankungen werden patientenbezogene Endpunkte immer wichtiger. So ist das Sistieren rektaler Blutbeimengungen bei Patienten mit Colitis ulcerosa (CU) ein international zunehmend akzeptierter Endpunkt in klinischen Studien und ein in der klinischen Praxis anzustrebendes Therapieziel. Wir untersuchten deshalb die Effektivität von Vedolizumab (VDZ), einem seit Mai 2014 zugelassenem monoklonalen Antikörper gegen alpha4-beta7-Integrine, bei Patienten mit CU.

Methodik: Aus 9 deutschen Behandlungszentren wurden 60 Patienten mit aktiver CU, die über 1 Jahr prospektiv zu Woche o, 2, 6, 14, 30 und 54 in einer Registerstudie dokumentiert, ausgewertet. Primärer Endpunkt war das Sistieren der rektalen Blutbeimengungen bei den täglichen Stuhlentleerungen in den vorangegangenen 7 Tagen vor der erneuten i.v. Gabe von 300 mg Vedolizumab. Parallel dazu wurde die Gesamtlebensqualität durch eine visuelle Analogskala (VAS; o-100) erfasst. Therapieabbrüche oder fehlende Daten wurden mit der Non-responder-Imputation (NRI) berücksichtigt.
Ergebnisse: $81,7 \%$ der Patienten waren mit Biologika (TNF-Antikörpern) vorbehandelt; $40 \%$ mussten in den letzten 12 Monaten vor der Behandlung mit VDZ hospitalisiert werden und $53,4 \%$ erhielten in Woche o Steroide (Median $20 \mathrm{mg}$, 0-60 mg/Tag). Zu Woche 54 berichteten $20 / 60$ Patienten $(33,3 \%)$ über ein Sisitieren der Hämatochezie; bei $17 / 60(28,3 \%)$ wurde dieser Endpunkt ohne begleitende Steroidmedikation erreicht. Zu Woche 54 nahmen 5 Patienten zusätzlich im Median $10 \mathrm{mg}$ Prednison/Tag ein. Folgende Ergebnisse zeigten sich zu Woche (W) \#: RB o / RB o und keine Steroide: W2: 25\%/10\%; W6: $36,7 \%$ / 23,3\%; W14: 43,3\% / 26,7\%; W30:36,7\% / 30,0; W54:33,3\% / 28,3\%. Die über die VAS erfasste Lebensqualität der Patienten stieg von " $47,5^{\prime \prime}$ (Woche o) auf "70,0" (Woche 14), "72,5" (Woche 30) und wiederum "70" (Woche 54) an.

Diskussion: Die Behandlung mit Vedolizumab führt bei Patienten mit schwer verlaufender Colitis ulcerosa und akuter Entzündungsaktivität in ca. 1/3 der Fälle zu einem raschen Sistieren der rektalen Blutbeimengungen. Dieses geht mit einer einer signifikant höheren Lebensqualität der Patienten einher. Langfristig kann dieser wichtige Endpunkt jedoch nur bei ca. 30\% der Patienten ohne begleitende Steroidmedikation erreicht werden.

\section{PS83}

Prevalenz von angeborenen Antithrombin Defiziten höher als erwartet

Dr. Ronald Fischer'; Dominik Eisenburger; Dr. Kathrin Heidinger; Prof. Bettina Kemkes-Matthes

${ }^{1}$ Universitätsklinikum Gießen und Marburg GmbH

Einleitung: Antithrombin (AT) ist ein wichtiger physiologische Inhibitor der Blutgerinnung. Die Prävalenz des hereditären AT-Mangels wird in der Bevölkerung mit 0,02 bis 0,2 \%, in selektierten Thrombosekollektiven mit bis zu $4 \%$ angegeben. Bei einigen Patienten mit thrombembolischen Ereignissen, die eine therapeutische Therapie mit niedermolekularen Heparinen erhielten, konnten wir eine niedrigere anti Faktor Xa aktivität nachgewiesen werden, als zu erwarten wäre. Bei diesen Patienten wurde eine Antithromin Genanalyse veranlasst. Trotz normaler Antithrombin Aktivität konnten Antithrombin Mutationen nachgewiesen werden. Daher könnte die Prävalenz von Antithrombin Mutationen höher sein, als angenommen.

Methoden / Patienten: Eine Antithrombin Genanalyse wurde bei 150 Patienten mit thrombembolischen Ereignissen ohne nachgewiesene schwere Thrombophilie (Faktor V-Leiden Mutation, Prothrombin Mutation, Protein C Mangel, Protein S Mangel) veranlasst. Die AT-Aktivität war normal auf einen Thrombin basierenden Test (Berichrome AT III (A) ${ }^{\circledR}$ von SIEMENS). Die Kontrollgruppe bestand aus 150 zur Blutspende zugelassenen Personen ohne thrombembolische Ereignisse. Auch dort wurde eine Antithrombin Genanalyse veranlasst.

Ergebnis: In der Patientengruppe fanden wir 6 Mutationen, trotz einer normal gemessenen Antithrombin Aktivität. Patient 1: Lungenembolie mit 21 Jahren. Antithrombin $72 \%$ Heterozygous missense mutation c.1248A>T ("Cambridge II"). Patient 2: tiefe Venenthrombose mit 24 Jahren. Antithrombin $106 \%$ Heterozygous missense mutation in exon 2 c.127A $>C$. Patient 3: Beckenvenenthrombose 39. Antithrombin $89 \%$ Heterozygous missense mutation in exon 2 c.89T>A. Patient 4: tiefe Venenthrombose mit 35Jahren. Antithrombin $88 \%$ of n.. Heterozygous missense mutation in exon 2 c.236G $>$ A. Patient 5: Lungenembolie mit 24 Jahren. Antithrombin $85 \%$ Heterozygous missense mutation in exon 7 C.1236G >T ("Cambridge II). Patient 6: mit 35 Jahren. Antithrombin $96 \%$ Heterozygous missense mutation in exon 2 c.89T>A. In der Kontrollgruppe wurde keine missense Mutation dedektiert. 
Fazit: 1. Die Prävalenz eines heriditären Antithrombin Mangels könnte höher sein als bisher angenommen. 2. Mit einem auf Thrombin basierenden funktionellen Antithrombin Test, lassen sich nicht alle Antithrombin Defekte erfassen.

\section{PS84}

Verbesserung des Impfstatus von Lebertransplantierten durch eine schriftliche Information an Hausärzte

Anna Herwig'; Dr. med. Dorothea Dehnen'; Prof. Dr. med. Kerstin Herzer²; Prof. Dr. med. Birgitta Weltermann ${ }^{1}$

${ }^{1}$ Institut für Allgemeinmedizin, Universitätsklinikum Essen, Universität Duisburg-Essen; ${ }^{2}$ Klinik für Allgemein-, Viszeral- und Gefäßchirurgie sowie Klinik für Gastroenterologie und Hepatologie des Universitätsklinikums Essen

Hintergrund: Bei Patienten nach Organtransplantation (SOT) besteht ein erhöhtes Infektionsrisiko. Bspw. ist die jährl. Inzidenz von Pneumokokkeninfektionen unter SOT 12,8-mal höher als in der Allgemeinbevölkerung; die höchste Rate haben Lebertransplantierte (354/100.000 Lebertransplantierte/Jahr) [1]. Impfungen können solche lebensbedrohlichen Krankheiten verhindern. In einer Querschnittsstudie zeigten wir, dass nur 0,3 \% der Lebertransplantierten unseres universitären Zentrums einen vollständigen Impfstatus nach STIKO hatten [2]. In dieser Studie untersuchten wir die Wirkung einer schriftlichen Information an Hausärzte.

Fragestellung: In welchem Umfang verbesserte eine Lebertransplantations-spezifische Impfinformation, die gemeinsam mit dem Arztbrief einer universitären Lebertransplantationsnachsorgeambulanz versendet wurde, die Prävalenz von Standard- und Indikationsimpfungen dieser Hochrisikopatienten?

Methodik: Die Hausärzte aller Lebertransplantierten, die an der o. g. Studie zum Impfstatus teilgenommen hatten, erhielten im Zeitraum vom 1.1.2014 bis 31.12.2015 eine schriftliche Impfinformation. Im Jahr 2016 wurden die Impfpässe dieser Patienten bzgl. Impfungen der vergangenen beiden Jahre durchgesehen. Die Prävalenzraten der Baseline- und der FollowUp-Studie für die Indikationsimpfungen (Pneumokokken, Hepatitis A/B, Influenza) und die Standardimpfungen (Tetanus, Diphtherie, Poliomyelitis, Pertussis) wurden miteinander verglichen.

Ergebnisse: Von 444 in die Studie eingeschlossenen Patienten waren $57,9 \%$ männlich; das durchschnittli-che Alter betrug 53,8 Jahre, das Alter bei Transplantation 46,1 Jahre. Die Prävalenz für Impfungen vor der Intervention waren: Pneumokokken 60,4 \%, Hepatitis A 23,4\%, Hepatitis B $41 \%$, Influenza 26,6 \%, Tetanus 41,5\%, Diphtherie 32,1\%, Poliomyelitis $15,1 \%$, Pertussis $39,4 \%$. Im Interventions-zeitraum wurden $40,5 \%$ der LT mindestens einmal geimpft. Dabei verbesserten sich die Prävalenzen der folgenden Impfungen: Pneumokokken $+7,8$ $\%$, Hepatitis A $+7 \%$, Hepatitis B $+6,5 \%$, Tetanus $+3,5 \%$, Diphtherie $+3,3$ $\%$, Poliomyelitis $+1,3 \%$, Pertussis $+8,6 \%$. Einzig die saisonale Impfrate für Influenza verringerte sich um - 5,4\%.

Diskussion: Über $40 \%$ unserer Lebertransplantierten wurden im Beobachtungszeitraum geimpft, was als Erfolg zu bewerten ist. Doch ist kritisch zu sehen, dass bspw. trotz des hohen Risikos für eine Pneumokokkeninfektion über $30 \%$ der Patienten unverändert nicht gegen Pneumokokken geimpft sind. Derzeit entwickeln wir eine intensivierte Intervention mit einem personalisierten Anschreiben für Ärzte und Patienten.

[1] Kumar D, Humar A, Plevneshi A et al: Invasive pneumococcal disease in solid organ transplant recipients - 10-year prospective population surveillance. Am J Transplant, 2007; 7(5): 1209-14.
[2] Weltermann B, Herwig A, Dehnen D, Herzer K: Vaccination Status of Pneumococcal and Other Vaccines in 444 Liver Transplant Patients Compared to a Representative Population Sample. Ann Transplant 2016; 21:200-207.

\section{PS85}

Best-practices für die Impfstofflagerung: ein Review von nationalen Empfehlungen und Leitlinien verschiedener Länder (Keep Cool)

Anika Thielmann; Prof. Dr. Birgitta Weltermann

Institut für Allgemeinmedizin, Universitätsklinikum Essen, Universität Duisburg-Essen

Hintergrund: Die korrekte Lagerung und Kühlung von Impfstoffen ist eine Voraussetzung für die optimale Wirksamkeit und Verträglichkeit von Impfungen. In früheren Studien zeigten wir diesbezüglich Defizite in Hausarztpraxen: $16 \%$ der Praxen hatten Erfahrungen mit Kühlkettenunterbrechungen, $49 \%$ führten kein Temperatur-Logbuch und $21 \%$ nutzten keinen separaten Kühlschrank für Impfstoffe. Eine Temperaturüberwachung in 21 Praxiskühlschränken ergab, dass diese in $10,2 \%$ der Messzeit außerhalb des Zielbereichs $\left(2^{\circ} \mathrm{C}\right.$ bis $8^{\circ} \mathrm{C}$ ) lagen; einzelne Kühlschränke wichen bis zu 66,3\% der Messzeit vom Zielbereich ab. Derzeit gibt es keine detaillierten Empfehlungen zur Impfstofflagerung in Deutschland. Für die Entwicklung einer Web-basierten Fortbildung zur Impfstofflagerung wurden best-practices basierend auf nationalen Empfehlungen und Leitlinien verschiedener Länder formuliert.

Methodik: Als erster Schritt erfolgte ein Review US-amerikanischer, britischer, schottischer, kanadischer und australischer Leitlinien und Empfehlungen zum Impfmanagement mit Schwerpunkt auf der Lagerung von Impfstoffen. Die extrahierten Inhalte wurden in wörtlicher Zitierung tabellarisiert gegenübergestellt. Zwei Reviewer exzerpierten die für das deutsche Setting relevanten, angemessenen und anwendbaren Kernempfehlungen. Unterschiedliche Einschätzungen wurden diskutiert. Abschließend wurden die Kernempfehlungen strukturiert aufbereitet.

Ergebnisse: Die exzerpierten Kernempfehlungen betreffen die Bereiche (1) Impfstofftemperatur (Temperaturempfindlichkeit von Impfstoffen, Thermometermodelle, Thermometerhandhabung), (2) Kühlschrank (Kühlschrankmodelle, Temperaturzonen, Temperaturschwankungen), (3) Lagerung (separater Kühlschrank, Bestellmenge und -kriterien, Ordnungssysteme), (4) tägliches Monitoring (Temperaturkontrolle, Logbuchdokumentation, Inventarliste) und (5) Zuständigkeiten (Arzt, MFA). Pro Bereich wurden 5 Kernempfehlungen formuliert.

Diskussion: Basierend auf den Ergebnissen wird jetzt eine Web-basierte Fortbildung für Ärzte und Medizinische Fachangestellte zur langfristigen Verbesserung der Impfstofflagerung in Hausarztpraxen entwickelt. 


\section{Versorgungsforschung 2}

\section{PS86}

Analyse der stationären Versorgungsqualität von Patienten mit spontan bakterieller Peritonitis zwischen 2007 und 2016

Aleksandra Markendudis'; Martina Mai'; Dr. Philipp Reuken²; Prof. Dr. med. Andreas Stallmach ${ }^{2}$; Dr. Tony Bruns ${ }^{2}$

${ }^{1}$ Universitätklinikum Jena; ${ }^{2}$ Universitätsklinikum Jena

Hintergrund: Die spontan bakterielle Peritonitis (SBP) ist eine häufige und schwerwiegende Komplikation der dekompensierten Leberzirrhose. Die 2011 publizierte deutsche S3-Leitlinie empfiehlt eine zeitgerechte, risikoadaptierte empirische Antibiotika-Therapie, die Albuminsubstitution zur Prävention des Nierenversagens und die Kontrolle des Therapieansprechens durch eine Aszites-Re-Punktion.

Methoden: Retrospektive Analyse von hospitalisierten Patienten mit einer ersten Episode einer SBP in den Zeiträumen $04 / 2007$ bis 12/2011 (Zeitraum 1) und 01/2012 bis 01/2016 (Zeitraum 2) in unserem Zentrum.

Ergebnisse: 173 Patienten mit einer Nachbeobachtung von mehr als 72 Stunden wurden identifiziert. Patienten, die im Zeitraum 2 behandelt wurden, erhielten signifikant häufiger Piperacillin/Tazobactam $(32 \%$ vs. $13 \%)$ und seltener Cephalosporine der 3 . Generation ( $35 \%$ vs. $57 \%)$ zur empirischen Therapie der SBP $(\mathrm{P}=0.009)$ und wurden häufiger innerhalb von 72 Stunden diagnostisch re-punktiert ( $55 \%$ vs. $21 \%$; $\mathrm{P}=0.00001$ ) als im Zeitraum 1. Eine Eskalation der empirischen antibiotischen Therapie war bei 32\% der Patienten im Zeitraum 2 und $40 \%$ der Patienten im Zeitraum 1 erforderlich $(P=$ n.s.) und trat häufiger bei Patienten mit hohem CRP zur Diagnose $(P=0.004)$ und bei Kultur-positiver SBP $(P=0.045)$ ein. Das kumulative 28 -Tage-Überleben nach SBP verbesserte sich signifikant von $64 \pm 6 \%$ im ersten Zeitraum auf $78 \pm 4 \%$ im zweiten Zeitraum $(\mathrm{P}=0.047)$. Univariate Prädiktoren für Tod oder Lebertransplantation innerhalb von 28 Tagen nach Diagnosestellung waren Organdysfunktion (Bilirubin, INR, Kreatinin) und Inflammation (Leukozyten bei Diagnose und bei Re-Punktion), ein geringerer Abfall der Neutrophilenkonzentration im Aszites und die Notwendigkeit der antibiotischen Therapieeskalation innerhalb von 14 Tagen.

Schlussfolgerungen: Die stationäre Versorgungsqualität von Patienten mit SBP hat sich innerhalb der letzten 10 Jahre signifikant verbessert.

\section{PS87 \\ Immunhistochemische Expression von Diaminoxidase (DAO) am oberen Gastrointestinaltrakt (GIT) bei Gastrointestinal vermittelten Allergien (GMA) \\ Thomas Vasilakis'; Prof. Dr. med. Martin Raithel ${ }^{2}$; Dr. med. Anna Katharina Kluger $^{2}$; Dr. Alexander Missbichler ${ }^{3}$; Antje Carstensen”; Jürgen Kressel'; Gabriele Frost?; PD Dr. Alexander Hagel'; Prof. Dr. med. Ralf Joachim Rieker ${ }^{1}$ ${ }^{1}$ Universitätsklinikum Erlangen; ${ }^{2}$ Waldkrankenhaus St. Marien Erlangen; ${ }^{3}$ SCIOTEC Diagnostic Technologies GmbH; ${ }^{4}$ FROST Diagnostika GmbH}

Einleitung: Diaminoxidase (DAO) kann Histamin, der Hauptmediator bei allergischen Reaktionen, mittels oxidativer Desaminierung deaktivieren. Es wurde bereits nachgewiesen, dass die Aktivität der DAO im Colon bei Gastrointestinal vermittelter Allergie (GMA) erniedrigt ist. Ziele dieser Studie waren daher die Erforschung der topographischen Lokalisation von DAO am oberen GIT sowie der Vergleich der immunhistochemischen Expression von DAO am oberen GIT zwischen Personen mit und ohne GMA.

Methodik: Es handelt sich um eine retrospektive Studie mit 21 Patienten mit nachgewiesener GMA und 17 nahrungstoleranten Kontrollpatienten. Gewebeproben aus Ösophagus, Cardia, Corpus, Antrum und Duodenum wurden für DAO-Immunhistochemie gefärbt. Die Expression der DAO wurde semiquantitativ basierend auf der Fär- bungsintensität der DAO (FI-DAO) analysiert: o = kein Signal, $1=$ geringe, $2=$ mittlere und $3=$ starke Intensität (Rotfärbung). Diese Analyse erfolgte longitudinal vom Ösophagus bis zum Duodenum und vertikal vom Oberflächenepithel bis zur Submukosa ( 5 Schichten). Zwei Messungen erfolgten zu zwei unterschiedlichen Zeitpunkten vom gleichen Untersucher.

Ergebnisse: DAO fand sich in allen 5 Schichten, allerdings quantitativ unterschiedlich ausgeprägt. Am stärksten fand sich DAO in den Papillen der Lamina propria im Ösophagus sowie in der subepithelialen Lamina propria (unmittelbar unter der epithelialen Basalmembran) in den anderen Segmenten. Diese Verteilung war in beiden Gruppen gleich. Insgesamt war die FI-DAO in allen Segmenten von beiden Gruppen gering. Im Duodenum war aber die FI-DAO bei der GMA-Gruppe statistisch signifikant niedriger im Vergleich zur Kontrollgruppe (Median: 0,77 geg. 1,13; $p=0,036$ ). Die DAO-Aktivität im Plasma korrelierte mäßig mit der FI-DAO nur im Duodenum bei Patienten mit GMA $(r=0,5 p=0,068)$

Schlussfolgerung: Die topographische Lokalisation von DAO im Gewebe verstärkt die Annahme, dass die DAO extrazellulär wirkt und aus der Mukosa resorbierte biogene Amine vor ihrem Eintritt ins Gewebe deaktivieren soll. Die FI-DAO im Duodenum war bei Patienten mit GMA im Vergleich zur Kontrollgruppe signifikant reduziert. Dieses Ergebnis zeigt, dass bei GMA Histamin-vermittelte Symptome lokal am ehesten im Duodenum entstehen, was häufige Symptome von Dyspepsie und Reizdarm bei diesem Patientenkollektiv erklären kann. In Anbetracht von den oben genannten Ergebnissen könnte die DAO-Färbung im Duodenum als Ergänzung zur konventionellen Histologie zur Abklärung von Histamin-induzierten Erkrankungen beitragen.

\section{PS88}

\section{Screening auf obstruktive Ventilationsstörungen} bei HIV-infizierten Patienten

Carina Breitenmoser; Prof. Dr. med. Reinhold Ernst Schmidt; Prof. Dr. med. Georg M.N. Behrens; Dr. med. Alexandra Jablonka Medizinische Hochschule Hannover

Hintergrund: Die Prävalenz der chronisch obstruktiven Lungenerkrankung (COPD) nimmt weltweit zu. Erhöhte Prävalenzen der COPD bei HIV-infizierten Patienten werfen die Frage nach dem Nutzen eines systematischen Screenings in diesem Kollektiv auf.

Ziel: Untersuchung der Lungenfunktion in einer Kohorte aus HIV-infizierten Patienten zur Evaluation des Potenzials eines Screenings.

Methodik: Im Rahmen von Routinebesuchen in einer immunologischen Ambulanz in Hannover wurden bei 139 erwachsenen HIV-positiven Patienten im Oktober 2016 Lungenfunktionsuntersuchungen durchgeführt. Bei auffälligen Messparametern erfolgte zusätzlich eine postbronchodilatatorische Spirometrie. Von 139 durchgeführten Spirometrien standen $76 \%(n=106)$ bei suffizienter Qualität für die Analyse zur Verfügung.

Ergebnisse: Das mittlere Alter der Patienten betrug 50 \pm 11 Jahre. 79,3\% waren männlich, ein Patient transgender. Die Patienten stammten aus 15 verschiedenen Ländern, darunter waren $87 \%$ kaukasischer, $8 \%$ afrikanischer und $5 \%$ asiatischer Herkunft. Im Mittel waren die Patienten $1,76 \pm 0,09 \mathrm{~m}$ groß und wogen $79,1 \pm 14,8 \mathrm{~kg}$. Die mittlere forcierte Vitalkapazität (FVC) belief sich auf 4,39 $\pm 0,94 \mathrm{l}$, was im Mittel 96\% des errechneten Sollwerts entspricht. Die mittlere Einsekundenkapazität (FEV1) lag bei 3,23 $\pm 0,78$ I (89\% Soll). Für den Tiffeneau-Index (FEV $1 /$ FVC) ergab sich im Mittel ein Wert von 0,74 $\pm 0,1$ (93\% Soll). 27\% ( $n=29)$ der gültigen Spirometrien zeigten abnorme Ventilationsparameter. In 22\% (23/106) der Fälle lag eine obstruktive Ventilationsstörung vor, während $6 \%(n=6)$ restriktiv verändert waren. $17,7 \%$ der obstruktiven 
Ventilationsstörungen waren reversibel. Der mittlere Tiffeneau-Index der Patienten mit Bronchialobstruktion nach Reversibilitätstestung betrug $0,57 \pm 0,09$ ( $73 \%$ des Sollwerts). Nach Verabreichung eines Bronchodilatators belief sich die mittlere FEV 1 der Patienten mit obstruktiver Ventilationsstörung auf 2,39 \pm 0,5 I (64\% Soll).

Schlussfolgerung: Der hohe Anteil an Patienten mit einer obstruktiven Ventilationsstörung bestätigt eine erhöhte COPD Prävalenz. Besonders alarmierend ist die niedrige Einsekundenkapazität der $\mathrm{Pa}$ tienten mit Bronchialobstruktion, die einer mittelschweren Obstruktion nach GOLD entspricht. Bei HIV-infizierten Patienten scheint ein Screening auf Atemwegserkrankungen mittels Spirometrie lohnenswert, um gezielte Maßnahmen zur Primär- sowie Sekundärprävention ergreifen zu können.

Die Arbeit wurde unterstützt durch die „Else-Kröner-Fresenius Stiftung" im Rahmen des KlinStrucMed.

\section{PS89 \\ Produktprobleme bei implantierbaren Arzneimittelpumpen und deren Zubehör - Analyse der 2005 - 2016 vom BfArM veröffentlichten Kundeninformationen \\ PD Dr. med. habil. Rüdiger Siekmeier'; Dipl.-Bio. Jürgen Hannig'; Prof. Dr. med. Kurt Rasche ${ }^{2}$ \\ ${ }^{1}$ Pharmazeutisches Institut der Universität Bonn; ${ }^{2}$ Bergisches Lungenzent- rum, HELIOS Klinikum Wuppertal}

Inverkehrbringen und Marktüberwachung von Medizinprodukten sind in Europa durch Europäische Direktiven geregelt. Bei Vorkommnissen und korrektiven Maßnahmen (Field Safety Corrective Actions, FSCA) müssen Hersteller die Behörden (Competent Authority, CA; in D: BfArM) informieren und diesen und Kunden entsprechende Kundeninformationen (Field Safety Notices (FSN) zur Verfügung stellen. Diese Arbeit analysiert FSCA zu implantierbaren Arzneimittelpumpen und deren Zubehör, die zwischen Anfang 2005 und September 2016 vom BfArM auf der Homepage publiziert wurden. Nach Ausschluß von Follow-up Meldungen fanden sich 37 FSCA/FSN von denen 27 Pumpen und Steuergeräte und 10 Katheter betrafen. Anwendungsgebiete der meisten Devices waren die intrathekale Schmerztherapie (z. B. Morphin bei Tumorpatienten) und die intrathekale Spastiktherapie (z. B. Baclofen bei Paraplegikern), während andere Indikationen (z. B. Prostazyklin bei pulmonaler Hypertonie) zahlenmäßig von untergeordneter Bedeutung waren. Typische Produktprobleme bei Pumpen waren Materialfehler (z. B. Korrosion, Sensor- oder Motorversagen), Softwarefehler (z. B. Fehler der Kommunikation zwischen Pumpe und externem Steuergerät, fehlerhafte Flußberechnung) und Handhabungsprobleme (z. B. Verabreichung des Medikamentes in die Pumpentasche), die mit dem Risiko einer Über- oder Unterdosierung (teils auch beides bei Vorliegen des gleichen Produktfehlers) mit entsprechenden klinischen Symptomen führten, wobei Todesfälle jedoch nur selten berichtet wurden. Produktprobleme bei Kathetern waren typischerweise Material- oder Produktionsfehler mit Auftreten von Verstopfungen (Risiko einer Unterdosierung), Infektionen oder Blutungen. Zur Risikoreduktion erfolgten seitens der Hersteller bei Pumpen meist eine Kundeninformation zur Vermeidung des Produktproblems (z. B. zusätzliche Kontrollen, spezielle Handhabungsanleitungen) und in wenigen Fällen eine Empfehlung zur Explantation, vorübergehender Vertriebsstopp und Devicemodifikation. Bei Kathetern erfolgte hingegen typischerweise die Durchführung eines Rückrufes (mit obligater Kundeninformation) sowie Kundeninformationen zur Vermeidung des Produktproblems (z. B. spezielle Handhabungsanleitungen). Zusammenfassend zeigt sich, daß implantierbare Arzneimittelpumpen und deren Zubehör eine wichtige Produktgruppe bei der Versorgung der entsprechenden Patienten darstellen und wesentliche Unterschiede zwischen Geräten und Zubehör bestehen.
Im Falle von Produktproblemen und FSCA sind FSN zur Risikominimierung von großer Bedeutung.

\section{PS90}

Implementing an Electronic Clinical Handover System in a University Teaching Hospital: Physician Attituted and Clinical Outcomes

Dr. Thomas Karol Mross; Dr. John Joseph Coughlan; Dr. Richard Liston University Hospital Kerry

Introduction: Clinical Handover is defined as inter-clinician communication occurring at care interfaces. With the advent of the European Working Time Directive (EWTD) and the following decrease in hours worked, the number of handovers has increased proportionally. This has raised concerns about continuity of care and potential for patient safety to be compromised. Clinical handover has been identified as a major preventable cause of harm.

Short description of practice change implemented: In this study, we analyse the clinical outcomes and physician attitudes associated with the implementation of a electronic clinical handover system in our medical department.

Aim and theory of change: The aim of this project was to create a reliable, standardised, reproducible method of communicating information about our patients to other physicians.

Targeted population and stakeholders Target Population: Patients under the care of the medical department. Stakeholders: Doctors in the Medical Department. Clinical Director. General Manager. Timeline Aug 2016: General Manager and Clinical Director confirmed clinical handover as a priority for a quality improvement initiative. AugSep 2016: Staff Education on Importance of handover and distribution of handover template and protocols to all staff. Sep-Nov 2016: 6 week Handover Pilot. Nov-Now: Pilot results disseminated and standardised electronic handover project is continuing. We audit once monthly with results sent to staff.

Comments on sustainability: The process will be audited weekly (selected randomly once per month) to maintain standards and compliance.

Comments on transferability: Utilising only a simple word document \& a protocol for its use, we created a highly transferable cost neutral solution, to facilitate standardised electronic handover of patients to on call staff.

Conclusions: Simple template and protocol resulted in $\sim 5.3$ patients being handed over per night. Compliance with ICU/CCU patient handover averaged at 58.9\% over the 6 week pilot. (Range 33-89\%) At our current level of compliance, this simple initiative would result in $>1600$ patient handovers per year in our department, promoting continuity of care. Survey Data found the process to be highly acceptable to doctors. The handover protocol was felt to standardise the process $(23.5 \%$ vs $81.25 \%, p=0.007595)$ and increased physician confidence that tasks handed over would be performed $(17.6 \%$ vs $81.25 \%$, $\mathrm{p}=0.000943)$. Physicians reported increased satisfaction with handover $(23.5 \%$ vs $81.25 \%, p=0.000914)$ and the process did not result in the increased workload predicted ( $64 \%$ vs $6.25 \%, p=0.000485)$.

Discussions: Standardisation of Clinical Handover through utilisation of electronic templates. Potential for dedicated software solutions.

Lessons learned Our study demonstrates: electronic handover systems are feasible to implement in the Irish healthcare system. They 
are highly acceptable to physicians and have the potential to improve continuity of care.

\section{PS91 \\ Effect of $\mathrm{ABO}$ blood group on Ristocetin induced platelet function in patients with von Willebrand's disease}

Dr. Kathrin Heidinger; Dr. Ronald Fischer; Mohammed Alrifai;

Prof. Dr. Bettina Kemkes-Matthes

Introduction: von Willebrand Factor (VWF) levels are influenced by the $A B O$ blood group. VWF:Ag remains lower mean levels on blood group (BG) O than BG Non O. Diagnosis of von Willebrand disease (VWD) can be difficult. This raised the question of whether ABO blood groups holds true for ristocetin- induced aggregation of platelets as well. In this study platelet function of patients with VWD and healthy subjects was compared by means of Born aggregation. Both collectives were divided into BG o and Non-o subjects.

Methods: 87 patients with VWD were included (61 women, 26 men. age 18 to 61 years). A control group with 76 healthy subjects (21 women, 55 men) was included. Blood group, vWF:Ag, Ristocetin cofactor, platelet function using Born Aggregation were performed, MCMDM-1VWD bleeding questionnaire was administered. Subjects with signs of inflammation or intake of platelet aggregation inhibitors were excluded.

Results: BG Non O was in $49 \%$ and BG O in $51 \%$ of subjects. VWF:Ag in healthy subjects with BG O was significantly lower with $82 \%$ versus $116 \%$ of normal. VWF:Ag in patients with VWD with BG O $(62 \%$ of normal) was lower than in healthy subjects and lower than in patients with Non O blood group (70\% of normal). No significant difference occurred in ristocetin induced aggregation in VWD (93\% of normal) compared to controls (101\%). Further subgroup analysis revealed no difference.

Conclusions: Platelet function test showed no difference between VWD patients with BG blood group $\mathrm{O}$ und patients with BG Non O. Therefore, hypothesis that platelet function in VWD-patients with blood type o should be reduced from the one found in patients with Non-o, could not be confirmed.

\section{PS93}

Unterschiedliche Dosierungsschemata der neuen oralen Antikoagulantien erschweren deren Anwendung bei chronisch Nierenkranken im klinischen Alltag

Dr. med. Insa Emrich; Linda Feuer; PD Dr. Sarah Seiler-Mußler; Dr. Adam M. Zawada; Prof. Dr. med. Danilo Fliser; Prof. Dr. med. Gunnar H. Heine

Chronisch nierenkranke Patienten gehören zu einer kardiovaskulären Hochrisikogruppe, deren Therapieoptionen adäquat ausgeschöpft werden sollten. Oft ist dabei eine Antikoagulation indiziert. Unlängst ist es möglich neue orale Antikoagulation zu verwenden, darunter Rivaroxaban, Apixaban und Edoxaban. Da Medikamente bei eingeschränkter Nierenfunktion akkumulieren, ist eine Dosisanpassung notwendig. Pharmafirmen bestimmen dabei die Kreatininclearance mithilfe der Cockroft-Gault Formel, welche bereits seit Jahren durch die Abschätzung der glomerulären Filtrationsrate mittels der CKD-EPI Krea Gleichung abgelöst wurde. Unklar ist, inwieweit es zu Diskrepanzen bei der Dosierung von NOAKS bei chronischer Nierenerkrankung durch die unterschiedliche Bestimmung der Nierenfunktion (Cockroft-Gault Gleichung vs. CKD-EPI Krea Gleichung) kommt. Von 2008 bis 2015 wurden insgesamt 544 chronisch Nierenkranke der KDIGO-Stadien $\mathrm{G}_{2}-\mathrm{G}_{4}$ in die fortlaufende CARE FOR HOMe Studie eingeschlossen. Die Nierenfunktion zum Einschlusszeitpunkt wurde sowohl nach der Cockroft-Gault Gleichung als auch nach der CKD-EPI
Krea Gleichung bestimmt. Die Dosierungsschemata von Rivaroxaban $(\mathrm{KrCl}>50 \mathrm{ml} / \mathrm{min})$, Apixaban $(\mathrm{KrCl} 15-29 \mathrm{ml} / \mathrm{min}$, Kreatinin > 1,5 mg/dl, Alter $>80$, Körpergewicht $<60 \mathrm{~kg})$ und Edoxaban $(\mathrm{KrCl} 15-95 \mathrm{ml} / \mathrm{min})$ erfolgten nach den offiziellen Fachinformationen. 435 Patienten, die mit Rivaroxaban behandelt werden könnten, unterschieden sich nach Cockroft-Gault und CKD-EPI Krea nach den Dosierungsschemata nicht. 2 Patienten hatten eine eGFR $<15 \mathrm{ml} / \mathrm{min} / 1.73 \mathrm{~m} 2$ nach CKD-EPI Krea, und bekamen dadurch keine Antikoagulation, 12 wurden nach CKD-EPI überdosiert, und 95 nach CKD-EPI unterdosiert. Bei Apixaban betraf es 496 Patienten, die sich nach Cockroft-Gault und CKD-EPI Krea nicht unterschieden. 2 Patienten bekamen keine Antikoagulation, da sie eine eGFR $<15 \mathrm{ml} / \mathrm{min} / 1.73 \mathrm{~m} 2$ nach CKD-EPI Krea aufwiesen und keine zusätzlichen Kriterien erfüllten, ein Teilnehmer wurde nach CKD-EPI überdosiert, und 45 nach CKD-EPI unterdosiert. 361 Teilnehmer, die mit Edoxaban behandelt werden könnten, unterschieden sich nach Cockroft-Gault und CKD-EPI Krea nicht. 2 Patienten hatten eine eGFR $<15 \mathrm{ml} / \mathrm{min} / 1.73 \mathrm{~m} 2$ nach CKD-EPI Krea, und bekamen dadurch keine Antikoagulation, 12 wurden nach CKD-EPI überdosiert, und 91 nach CKD-EPI unterdosiert, 78 hatten nach der Cockroft-Gault Gleichung eine Kreatininclearance $>95 \mathrm{ml} / \mathrm{min}$, sodass die Behandlung mit Edoxaban kontraindiziert ist. Zusammenfassend wird die Diskrepanz zwischen der Bestimmung der Nierenfunktion nach Cockroft-Gault und CKD-EPI Krea im Hinblick auf die Dosierungsschemata der neuen oralen Antikoagulatien deutlich. Am ehesten ist dabei Apixaban zu empfehlen, dessen Dosierungsschemata neben der Nierenfunktion noch weitere Kriterien berücksichtigt. Fast $16 \%$ der chronisch nierenkranken Menschen haben alleine aufgrund unterschiedlicher Bestimmungen der Nierenfunktion keine Möglichkeit von einer antikoagulatorischen Therapie zu profitieren. " 
Der Internist · Suppl 1 · 2017 S43 
A

Ahmad, S., PS74

Ahnis, A., PS19

Albers, F., PS47

Alrifai, M., PS91

Alscher, M., PS64

Altenhofer, J., PS57

Ammar, N., PS35

Amr, A., YIA 1

Arlt, A., PS35

Aschenbrenner, E., PS27, PS29,

PS30

Athanasiadis, A., PS12

Atreya, R., PS82

\section{A}

Baeres, F., PS04

Baerwald, C., YIA 6, PS 54

Baier, K., PS37

Baier, M., PS24

Bailey, T., PS02

Bajrovic, A., PS75

Bals, R., PS32

Bamberger, C., PS57

Baraki, H., PS77

Bartels, C., PS77

Bauer, B., PS68

Bauer, L., PS24, PS60

Baumgart, D., PS82

Behrens, G., PS88

Benck, U., PS66

Benedix, D., PS09

Bergenstal, R., PSO4

Berghäuser, K., PS58

Bernhard, N., PS32

Betsch, B., PS69

Blaschke, S., PS48

Blüher, M., PS71

Bode-Böger, S., PS21

Böhm, M., PS76

Borggrefe, J., YIA 2

Borkenstein, D., PS81

Borte, G., PS78

Bosch-Traberg,, H., PS03

Bozec, A., PS13

Bradford, E., PS47

Braisch, U., PS73

Bramlage, P., PS01

Braun, N., PS64

Breitenmoser, C., PS88

Brown-Frandsen, K., PSO4

Bruns, T., PS25, PS82, PS86

Buchmann, N., PS05, PS33

Buhl, R., PS79

Bürger, M., PS36

Buse, J., PS04

Buss, S., PS67

Buttlar, J., PS34
Cabrera, M., PS66

Capellino, S., PS18

Carstensen, A., PS87

Chatzikyrkou, C., PS22

Chavanon, M., PS48

Christensen, S., PSO2

Churin, Y., PS37, PS 62

Cockle, S., PS47

Cohen-Solal, A., PS72

Cornberg, M., PS50

Coughlan, J., PS90

Crieé, C., PS79

D

Dallmeier, D., PS73

Daniels, G., PS04

Danne, T., PS01

Dechant, F., PS63

Decker, S., PS06

Dehnen, D., PS84

Delistefani, F., PS59

Demirci, C., PS63

Demuth, I., PS05, PS26, PS33

Denkinger, M., PS73

di Giuseppe, R., YIA 2

Dieterich, C., YIA 1

Dignass, A., PS82

Dinges, L., PS35

Divchev, D., PS42

Duca, I., PS38

Dumbrava, D., PS20

Dumitrascu, D., PS38

Duyster, J., PS39

Dührsen, U., YIA 3

Dybkova, N., PS74

E

Egger, J., PS07

Ehlermann, P., YIA 1

Eisenburger, D., PS83

Elbelt, U., PS19

Emrich, I., YIA 4, PS17, PS21, PS24,

PS60, PS72, PS93

Ende, K., PS82

Engel, R., PS65

Engelhardt, M., PS39

Espinola-Klein, C., PS69

$\mathbf{F}$

Fabian, A., PS34

Fähndrich, S., PS32

Feuer, L., PS72, PS93

Fischbach, F., PS09

Fischer, R., PS83, PS91
Fischer, T., PS74

Fliser, D., YIA 4, PS17, PS21, PS24,

PS60, PS72, PS93

Frank, M., PS46

Franke, A., PS75

Frankenstein, L., PS67

Frese, K., YIA 1

Frey, N., YIA 2, PS74, PS75

Frille, A., PS78

Frommer, K., PS65

Frost, G., PS87

\section{G}

Gastinger, I., PS11

Geier, C., YIA 1

Georg, C., PS69

Geroldinger, A., PS53

Gollasch, M., PS26, PS33

Gotthardt, M., YIA 1

Grabe, N., PS67

Grenkowitz, T., PS41

Greulich, S., PS64

Grünerbel, A., PS01

Gschwind, D., PS29, PS30

Gunsoy, N., PS47

H

Haas, J., YIA 1

Habbel, J., YIA 3

Hadem, J., PS77

Haderer, M., PS27

Hagel, A., PS87

Hagel, S., PS25

Hagendorff, A., PS78

Hahn, L., PS62

Halloul, Z., PS09, PS10

Hampe, J., PS82

Hann, A., PS07, PS23, PS40

Hannig, J., PS51, PS89

Hansen, M., PS77

Hanoun, M., YIA 3

Harsch, I., PS58

Hartmann, F., PS82

Hartmann, J., PS46

Hartmann, N., PS74

Hasenfuß, G., PS74

Hasper, I., PS56

Heckel, S., PS29

Heidinger, K., PS83, PS 91

Heine, G., YIA 4, PS17, PS21, PS24,

PS60, PS72, PS93

Heinemann, A., PS60

Heinsen, F., PS75

Heinze, G., PS53

Helm, O., PS34

Helmrich, N., PS62

Henes, J., PS64

Hengstenberg, C., PS28

Henze, K., PS57
Herebian, D., PS62

Herforth, M., PS52

Hermann, S., PS43

Herpel, E., PS67

Herr, M., PS77

Herrmann-Lingen, C., PS48

Herwig, A., PS84

Herzer, K., PS84

Hess, T., PS58

Hessse, L., PS35

Heymann, S., PS58

Hiller, H., PS48

Hofmann, T., PS19

Holl, R., PS01

Hollstein, T., PS41

Horstick, G., PS69

Hosang, S., PS77

Hülser, M., PS13

Husser, O., PS28

I

Illert, L., PS39

J

Jablonka, A., PS88

Jacob, S., PS03, PSO4

Jacobs, G., YIA 2

K

Kaesemann, P., PS64

Kaiser, M., PSO2

Kaltoft, M., PSO2

Kampmeyer, D., PS55

Kandulski, M., PS16, PS22

Kardos, P., PS79

Karges, W., PS01

Karimian, S., PS68

Kasel, M., PS28

Kassner, U., PS41

Kassubek, J., YIA 2

Kastrati, A., PS28

Katus, H., YIA 1, PS67

Katzenstein, J., PS11

Katzer, K., PS25

Kayvanpour, E., YIA 1, PS67

Keller, F., PS40

Keller, G., PS56

Kemkes-Matthes, B., PS83, PS91

Kentrup, D., PS43

Kern, S., PS60

Kern, W., PS02

Kircher, A., YIA 4

Kitterer, D., PS64

Klapp, B., PS19

Kluger, A., PS87 
Knaack, H., PS34

Koenig, W., PS73

Koch, M., YIA 2

Köhler, F., PS08

Köhler, M., PS39

Kohlweyer, U., PS39

König, M., PS26, PS33

Konturek, P., PS58

Köppel, A., PS37

Koziolek, M., PS48, PS59

Kragh, N., PS03

Krämer, B., PS66

Kressel, J., PS87

Kristensen, P., PSO4

Kübelbeck, A., PS43

Kümpers, P., PS49

Kunst, C., PS27, PS29, PS30

Kurrle, A., PS61

Kutschka, I., PS77

$\mathbf{L}$

La Rosee, P., PS08

Labenz, J., PS80, PS81

Lach, N., PS48

Lahrmann, B., PS67

Lai, A., YIA 1

Langbein, C., PS82

Lanzinger, S., PS01

Larbig, G., PS43

Laser, H., PS50

Latus, J., PS64

Launay, J., PS72

Lavall, D., PS76

Lechner, K., PS57

Leder, D., PS37

Legrand, M., PS72

Lenk, L., PS34

Leodolter, A., PS80

Lepper, P., PS32

Li, D., PS67

Liao, Y., YIA 1

Lieb, W., YIA 2, PS75

Lipovskiy, L., PS54

Lippert, H., PS11

Liss, M., YIA 1

Liston, R., PS90

Löffler, C., PS66

Lohse, M., PS30

Lorenz, H., PS56

Lossi, N., PS79

Lowin, E., PS48

Lüdde, M., PS75

Lüdemann, J., PS03, PS04

Luo, Y., PS13

Lutz, M., YIA 2

M

Maasoumy, B., PS50

Mahrholdt, H., PS64
Mai, M., PS86

Mailänder, C., PS79

Mair, R., PS63

Maislos, M., PSO2

Malysheva, O., YIA 6

Mann, J., PS03, PSO4

Manns, M., PS50

Markendudis, A., PS86

Marsche, G., PS60

Marso, S., PSO4

Martens-Lobenhoffer, J., PS21

März, W., PS41, PS51

Maul, J., PS82

Mavropoulou, E., PS48

Mayatepek, E., PS62

Mayr, A., PS64

Mayr, N., PS28

Mebazaa, A., PS72

Meder, B., YIA 1, PS67

Mertens, P., PS22

Messner, L., PS69

Meyer, F., PS09, PS10, PS11

Michel, J., PS28

Middendorf, J., PS27

Mikulits, W., PS34

Minnigerode, L., PS58

Missbichler, A., PS87

Mitas, D., PS45

Mittag, J., PS20

Mross, T., PS90

Müller, F., PS70

Müller, G., PS48, PS59

Müller, H., YIA 2

Müller, M., PS45, PS63, PS81

Müller-Ladner, U., PS13, PS18.

PS65

Müller-Schilling, M., PS27, PS29,

PS30

$\mathbf{N}$

Nauck, M., PS03, PS04

Nelsen, L., PS47

Neumann, E., PS13, PS18, PS65

Neurath, H., PS48

Neurath, M., PS82

Nicula, N., PS38

Nikolovska Vukadinovic, A.

PS76

Nissen, S., PSO4

Norman, K., PS05, PS33

Nosalski, E., PS40

Nöthlings, U., YIA 2

0

Obbarius, A., PS19

Ong, P., PS12

Ott, S., PS75

Otto, R., PS11
S

P

Pabel, S., PS74

Parhofer, K., PS57

Patrascu, A., PS64

Pavel, V., PS63

Pavenstädt, H., PS43

Pawelski, H., PS43

Pellegrini, C., PS28

Petros, S., PS78

Pfeiffer, D., PS78

Pirozzolo, G., PS12

Plöthner, M., PS46

Pocock, S., PS03, PS04

Pollinger, K., PS27, PS29, PS30

Poulter, N., PS04

Preiß, J., PS82

Pribe-Wolferts, R., YIA 1

Ptok, H., PS11

$\mathbf{R}$

Rahn, S., PS34

Raithel, M., PS87

Rao, P., PSO2

Rasche, K., PS89

Rasmussen, S., PS04

Rauh, M., PS14, PS15

Ravn, L., PS04

Rehart, S., PS18, PS65

Reichardt, B., PS53

Reschke, K., PS16

Reuken, P., PS86

Reuter, H., PS01

Reuter, S., PS43

Rheude, T., PS 28

Ribbentrop, D., PS46

Rieck, M., PS02, PS03, PS04

Rieker, R., PS87

Ritter, M., YIA 2

Röcken, C., PS35

Roderfeld, M., PS37, PS62

Roeb, E., PS37, PS62

Rogacev, K., PS60

Röpke, M., PS16

Rose, M., PS19

Rossmeier, A., PS57

Rossol, M., PS71

Rothenbacher, D., PS73

Rüddel, J., PS36

Rühlemann, M., PS75

Rütten, A., PS22

Schäfer, H., PS34, PS35

Schäfers, M., PS43

Scharhag, J., PS24

Scharnagl, H., PS41

Schenk, L., PS49

Schett, G., PS13
Schilling, T., PS77

Schlosser, S., PS27, PS29, PS30

Schmalstieg, D., PS07

Schmelz, R., PS82

Schmid, S., PS63

Schmidt, C., PS36, PS82

Schmidt, H., PS20

Schmidt, R., PS88

Schneider, F., PS37

Schreiyäck, C., PS13

Schulz, H., PS80

Schulze, H., PS82

Schunkert, H., PS28

Schütte-Nütgen, K., PS43

Sebens, S., PS34, PS35

Sechtem, U., PS12, PS64

Sedaghat-Hamedani, F., YIA 1 ,

PS67

Seibert, M., PS32

Seiler, S., YIA 4

Seiler-Mußler, S., PS17, PS93

Selgrad, M., PS45

Sellier, A., PS17

Seufert, J., PS01

Seufferlein, T., PS23

Sheikh Rezaei, S., PS53

Shin, J., PS47

Siefert, O., PS54

Siegmund, B., PS82

Siekmeier, R., PS51, PS89

Sossalla, S., PS74

Spehlmann, M., PS75

Spira, D., PS05, PS33

Spoo, A., PS08

Stallmach, A., PS25, PS36, PS82,

PS86

Stark, R., PS57

Stegner, S., PS34

Stein, J., PS68

Steinberg, W., PS04

Steiner, R., PS11

Steinhagen-Thiessen, E., PS05,

PS26, PS33, PS41

Stengel, A., PS19

Stock, C., PS67

Stock, J., PS48

Stöckl, G., PS42

Stockner, M., PSO4

Surlan, I., PS39

Suruki, R., PS47

Sziegoleit, S., PS01

$\mathbf{T}$

Takacs, R., PS02

Taute, B., PS31

Teich, N., PS82

Tergast, T., PS50

Teupe, C., PS68

Thielmann, A., PS85

Thomas-Rüddel, D., PS36

Thrum, S., YIA 6, PS71

Thum, F., PS31

Tirilomis, P., PS74 


\section{Autorenregister}

Trenkwalder, T., PS28

Tschuschner, A., PS37, PS62

Tsiklauri, L., PS65

Tsoukas, G., PS02

Tugrul, F., PS67

$\mathbf{U}$

Udelnow, A., PS09

Untersteller, K., PS72

V

van Nie, L., PS18

Vassen, L., YIA 3

Vasilakis, T., PS87

Viol, F., PS34

Vodovar, N., PS72

Vogelmeier, C., PS32, PS79

von Arnim, U., PS82

Vukadinovic, D., PS76

W

Wagenpfeil, S., PS32, PS76

Wagner, U., PS71

Waldmann, E., PS57

Wallbach, M., PS48, PS59

Walter, M., YIA 5

Weber, M., PS14, PS15

Weibert, E., PS19

Weidhase, L., PS78

Weiher, M., PS45

Weise, D., PS01

Weise, R., PS80

Weltermann, B., PS84, PS85

Wenisch, S., PS65

Werner, J., PS65

Winkler, T., PS75

Wolff, S., PS11

Wolfrum, S., PS55

Wolzt, M., PS53

Worth, H., PS79

Wu, L., PS57

Z

Zawada, A., PS17, PS21, PS24,

PS60, PS 93

Zigan, S., YIA 5

Zinman, B., PS04

Zizer, E., PS23

Zoller, W., PS07

Zhu, F., YIA 1 

